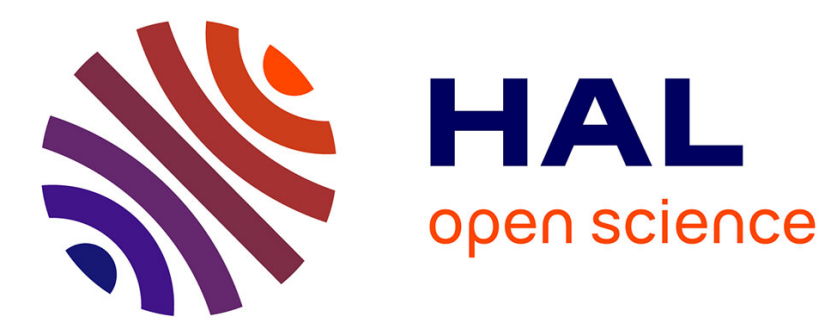

\title{
The reductive perturbation method and some of its applications
}

\author{
Hervé Leblond
}

\section{To cite this version:}

Hervé Leblond. The reductive perturbation method and some of its applications. Journal of Physics B: Atomic, Molecular and Optical Physics, 2008, 41 (4), pp.043001. 10.1088/0953-4075/41/4/043001. hal-03423758

\section{HAL Id: hal-03423758 \\ https://univ-angers.hal.science/hal-03423758}

Submitted on 10 Nov 2021

HAL is a multi-disciplinary open access archive for the deposit and dissemination of scientific research documents, whether they are published or not. The documents may come from teaching and research institutions in France or abroad, or from public or private research centers.
L'archive ouverte pluridisciplinaire HAL, est destinée au dépôt et à la diffusion de documents scientifiques de niveau recherche, publiés ou non, émanant des établissements d'enseignement et de recherche français ou étrangers, des laboratoires publics ou privés. 


\title{
TUTORIAL
}

\section{The reductive perturbation method and some of its applications}

\author{
Hervé Leblond \\ Laboratoire POMA, FRE 2988, Université d'Angers, 2 Boulevard Lavoisier, 49000 Angers, France
}

Received, in final form 12 December 2007

Published 11 February 2008

Online at stacks.iop.org/JPhysB/41/043001

\begin{abstract}
The reductive perturbation method is a very powerful way of deriving simplified models describing nonlinear wave propagation and interaction. In abstract frames chosen for the sake of clarity, we describe the fundamentals of the method: envelope equations, long-wave approximation, three-wave resonant interaction. We give an insight into the mathematical properties of the perturbative schemes. Then some applications are given, which either illustrate the typical situation or introduce additional features of perturbative expansions, and have their own physical interest. The applications concern either nonlinear optics, especially ultrafast, or wave propagation in ferromagnetic media, in the so-called electromagnetic or polariton range.
\end{abstract}

(Some figures in this article are in colour only in the electronic version)

\section{The reductive perturbation method}

\section{1. 'Weakly' nonlinear}

Nowadays, research in physics devotes much attention to nonlinear phenomena. What is the reason for this? In fact, most physical phenomena are intrinsically nonlinear. Newton's gravitational attraction law involves a spatial dependency as $1 / r$, the pendulum equation is transcendent, considering the most elementary examples only.

However, the mathematical techniques used in the study of these problems remained linear for a long time. It has long been considered that two quantities were dependent on each other when they were proportional only, i.e. any nonlinear dependency was ignored. This happened because the mathematics of the time was not able to yield a better theory. Since then, many techniques of mathematical analysis have been developed, often more successfully for linear problems. As a fundamental example, the resolution of sets of linear algebraical equations is well known, while a polynomial equation of more than fourth degree cannot be solved analytically in general. Regarding differential equations, the same observation is made: if the equation or the system is linear, Fourier analysis allows us to reduce it to algebraical equations, which allows us to solve a large part of the problems. Analogously, most of the problems involving linear differential equations can be solved by means of Fourier analysis, while for nonlinear differential equations no equivalent procedure exists, and mathematical difficulties are numerous.

To overcome these shortcomings in the mathematics, physics has made use, in an almost systematical way, of approximations that allow us to replace the initial mathematical problem, nonlinear and not solvable, by another one, linear and solvable. The small oscillations approximation for the pendulum is a typical example. For some applications, an intrinsically nonlinear physical system is put in a special situation, for which a linear approximation can be used (e.g. a transistor, in analogical electronics). The same approach is used in the study of electromagnetic waves in ferromagnetic media: the sample is immersed in a strong enough external magnetic field. An important exception to this general scheme is constituted by electromagnetic waves, whose behaviour, in vacuum, seems to be exactly linear, insofar as this makes sense. For visible frequencies in a transparent medium, and for all sources existing on Earth before the laser was created, the linear approximation is valid. Here, technological progress allowed us to attain domains where this approximation is no longer acceptable. 
The physical concepts related to the study of linear or linearized phenomena are intimately related to mathematical objects that make sense for linear problems only: frequency, polarization, mode, .... The most natural approach, which is also the most affordable technically, is to use a perturbation method, the fist order of which is the linear approximation: the slowly varying envelope approximation, typically. It can thus be qualified as 'weakly nonlinear', in accordance with the terminology of mathematicians.

It must be noticed that, if some evolution law is not linear, the Fourier components are not conserved. Even if the Fourier transform can be mathematically defined at any time, this lack of conservation destroys its physical meaning. The physical sense can be given by the trace of the properties of the linearized system only. It is thus necessary to avoid going too far away from the latter, i.e. to use a weakly nonlinear approximation, in the abovementioned sense. Further, and especially in nonlinear optics, the weakly nonlinear approximation may be fully justified on experimental grounds, without the necessity for producing a special situation.

However, many phenomena, although they are weakly nonlinear in the sense that the frequency and so on still have a meaning, diverge in their results from the linear situation. This is due to the fact that small modifications of an evolution law can yield large variations, if the evolution time is long enough. In this frame, the multiscale analysis is fully justified: it is a perturbative approach, whose first order is a linear approximation, that allows us to write not small corrections valid for the same evolution time as in the linear approximation, but their cumulative effect for a very long evolution.

\subsection{Soliton theory}

The reductive perturbation method is intimately related to soliton theory. It is indeed well known and commonly used by the specialists of the latter. Before we expose the fundamental reasons for this association, let us explain what a soliton is.

The first observation of a soliton was experimental, as far back as 1834, and due to J Scott Russell. Riding alongside a canal, he observed 'a large solitary elevation, a rounded, smooth and well-defined heap of water, which continued its course along the canal apparently without change of form or diminution of speed', and which was 'preserving its original figure' for a very long distance [1]. (The full passage can be found in many papers and books on soliton theory and encyclopaedias.). It is thus a solitary wave, i.e. here a single hump of water, which propagates without deformation. In a linear non-dispersive medium, propagation without deformation occurs for any wave. But if dispersion is present, only a purely sinusoidal wave can propagate without deformation; a wave packet always spreads out, a fortiori does a solitary wave. If a nonlinear effect is present, it always results in wave deformation, frequently in wave breaking [2]. However, the nonlinear and dispersive effects can compensate together for a special wave shape: this is the soliton.

The theory of hydrodynamic solitons began in the 19th century first with Boussinesq, who published in 1872 the equation which has his name, which is a version of the well-known Korteweg-de Vries (KdV) equation allowing the propagation in both directions (while $\mathrm{KdV}$ is unidirectional) [3]. Boussinesq solved this equation and gave the expression of the $\mathrm{KdV}$ soliton 23 years before Korteweg and de Vries themselves [4]. The KdV equation was fully solved much later in 1967; however this resolution was again a pioneer work in soliton theory, since it involved the construction of the so-called inverse scattering transform (IST) method [5]. In short, there is some mathematical transformation, called the inverse scattering transform (IST) [6, 7], that generalizes the Fourier transform and allows us to solve the Cauchy problem. The method uses the resolution of an inverse problem, which motivates its appellation. This scheme can be achieved for a small set of nonlinear partial differential equations only, among which is the KdV. These equations are called integrable (or completely integrable). A mathematical property which renders an equation integrable is the existence of a Lax pair [8]. Further, integrable equations have the remarkable property of possessing an infinite number of conservation laws.

A second type of soliton was discovered later: the envelope soliton. In the same way as a single hump, a wave packet involving some carrier wave modulated by an envelope can be stabilized by the combined effect of dispersion and nonlinearity. In this case the theory came first [9], with the experiment only eight years later [10]. It demonstrated the propagation of picosecond optical pulses in a fibre. The model equation is here called the nonlinear Scrhödinger equation (NLS); it is completely integrable.

The same mathematical model describes the diffraction of a narrow beam in a planar optical waveguide filled with a medium which presents the same cubic Kerr nonlinearity as silica fibre. According to exactly the same mathematical properties of the model, the stabilization of the beam occurs: it is the spatial soliton, first observed in 1985 [11].

Thereafter the concept of the soliton has been widely extended; a few examples will be given in this tutorial.

\subsection{Multiple scales}

A first close link between multiscale expansions and integrable equations comes from the physical situations in which the latter arise. Let us consider the KdV equation in hydrodynamics: the formation of solitons assumes relations to be satisfied between the amplitude of the solitary waves, their lengths, the canal depth and the propagation distance. All these quantities are lengths, whose orders of magnitude differ, but are in no way arbitrary. From the physical nature of the phenomenon itself, we have multiple scales. Regarding the envelope solitons, let us consider, e.g., optical solitons in fibres. The wavelength, the pulse length and the propagation distance are three lengths measured along the fibre axis that are first distinguished by their different orders of magnitude. Further, the formation of a soliton occurs only if some relations are satisfied between these orders of magnitude, and between them and that of the wave amplitude. Here again, multiple scales exist in the physics itself.

The formalization of the multiple scales involves the introduction of some small perturbation parameter $\varepsilon$, so that 
the orders of magnitude of the various effects are determined by means of their order in an expansion in a series of powers of $\varepsilon$. This induces homogeneity properties of the mathematical expressions considered. Therefore, the model equations which are derived this way must satisfy these homogeneity properties: the number of possible equations is hence rather small. Consequently, a few equations are shown to account for analogous phenomena in many very different domains of physics. Such equations are called universal. An important number of them are completely integrable, although this property is very rare. This relates closely the multiscale analysis or reductive perturbation method to soliton theory.

It has also been shown [12] that the reductive perturbation method, when applied to some integrable equation, could lead to some other equation, again integrable. This has been (and is still) used to construct approximate solutions of integrable equations and to study the mathematical properties of the latter (see the short review in the physical frame in section 9.2).

The reductive perturbation method, or multiscale analysis, seems to be initially due to Gardner and Morikawa [13], concerning hydromagnetic waves in a cold plasma. The method was used again by Washimi and Taniuti [14], applied to the study of ion-acoustic waves. Taniuti and Wei wrote a general theory of the derivation of the Korteweg-de Vries $(\mathrm{KdV})$ equation [15]. Let us also mention the derivation of the $\mathrm{KdV}$ and Burgers' equations, by Gardner and $\mathrm{Su}$ [16]. Taniuti and his co-workers initiated the method concerning the envelopes, leading to the nonlinear Schrödinger (NLS) equation. It is remarkable that the first paper in which this equation has been derived using the reductive perturbation method, i.e., a multiscale expansion, does not, properly speaking, study an envelope problem [17]. The phase of the complex variable satisfying this equation describes the precession of a magnetic field around the propagation direction. This situation, initially studied in a cold plasma, has been applied to a ferromagnetic dielectric ever since [18]. Taniuti and Yajima have given a 'perturbation method for a nonlinear wave modulation', deriving the NLS equation using a multiscale method, in two papers, the first one using as initial model a system of partial differential equations (PDEs) [19], the second one being generalized to the integro-differential systems [20].

Note that the reductive perturbation method is often referred to as the WKB (Wentzel, Kramers, Brillouin) one, especially in the mathematical community. However, the original WKB method approximates the stationary solutions to the (linear) Schrödinger equation, which quite differs from the problems we are considering here.

\subsection{A word about the convergence proofs}

Rigorously, a formal asymptotic expansion does not prove anything: it must be proved that the difference between the solution of the initial model and that of the asymptotic model becomes effectively very small as the perturbative parameter tends to 0 . This is called the convergence of the expansion. The convergence of multiscale expansions is not an easy problem and is far beyond the scope of this tutorial. Regarding the asymptotics of slowly varying envelope type, the first studied situation seems to be that of nonlinear geometrical optics [21, 22]: it is the situation where the nonlinear effect (selfphase modulation) is strong enough to arise at distances comparable to the length of the considered short pulse. The propagation of the envelope at group velocity is directly modified by this phenomenon. This situation is mathematically more simple than that of the NLS-type asymptotics, since it involves only two scales instead of three. It corresponds physically to higher intensities and shorter propagation distances. Once this was done, mathematicians began to study the three scale expansions and succeeded in justifying the NLS-type asymptotic in media with a cubic nonlinearity [23]. The next step concerns the NLS and DaveyStewartson (DS)-type asymptotics, in media with a quadratic nonlinearity [24, 25]. Long-wave asymptotics have also been justified, e.g., the convergence of the Burgers asymptotics for shock waves in ferromagnetic media has been recently proved [26]. The formal derivation had been published nine years previously [27].

From these convergence proofs, I note two details of great importance for physics. First, the convergence can only be justified on finite length intervals of the evolution variable, time or propagation distance depending on the point of view. This evolution variable corresponds to a particular scale that will be characterized by a typical length $L_{0}$. It means that, after a propagation distance of a few $L_{0}$, the NLStype approximation will no longer be valid. This accounts for the experimental fact that the spatial optical solitons 'do not exist' or, more exactly, that instead of propagating indefinitely without any deformation, as the corresponding solution of the NLS equation does, these structures break up after a few diffraction lengths. If the convergence of the asymptotic is taken into account, it is known that the exact solution of the Maxwell equations must diverge from that of the NLS after a few $L_{0}$. This is exactly what has been observed; the length of diffraction plays the role of the above characteristic length $L_{0}$. This qualitative remark was already conjectured in [19].

The second point that intervenes in these mathematical problems, and is retrieved in experiments, is the competition between the soliton formation and the diffusions. Studying experimentally spatial solitons in a planar waveguide, it has been noticed that the stimulated Rayleigh wing and Raman diffusions could occur together with the Kerr effect and modify in an important way the formation of solitons as much as the interactions between modes [28, 29]. The observed results depend on the relative intensity of the various nonlinear couplings: stimulated Rayleigh diffusion, stimulated Raman diffusion, self-phase modulation, cross phase modulation. A trace of this appears in the convergence proofs: indeed, Joly, Métivier and Rauch have proved that, under certain conditions, the convergence could be justified only if some conditions, called transparency conditions, were satisfied [30]. A rough interpretation of these transparency conditions is that all the diffusions can be neglected. It is remarkable that difficulties appearing in purely mathematical aspects of the question correspond very closely to experimental features. A complete analysis of this problem remains an open issue. 


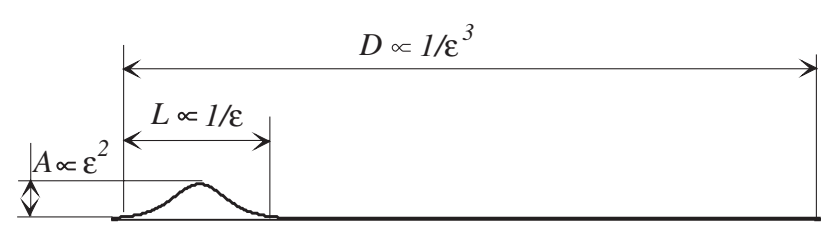

Figure 1. The various length scales of the $\mathrm{KdV}$ soliton. $A$ : small amplitude, $L$ : long wavelength, $D$ : much longer propagation distance.

\section{Long-wave expansions}

After these considerations about the history and the interest of the method, let us describe it more precisely, beginning with the most typical situations and with the long-wave approximation. One of the first studies devoted to this matter is [16], which derives the equations of Burgers and $\mathrm{KdV}$ for hydrodynamical waves in shallow water. The Burgers' equation describes an equilibrium between the nonlinearity and the dissipation, and the formations of smooth shock profiles. The $\mathrm{KdV}$ equation describes an equilibrium between the nonlinearity and the dispersion, and the formation of localized pulses propagating and crossing each other without deformation, called solitons. There exists an electromagnetic wave mode in ferromagnetic media which obeys either the Burgers' equation [27] or the KdV one [31], depending on the values of the wave amplitude and the damping constant.

We consider a solitary wave with long length $L$, weak amplitude $A$, propagating on a distance $D$, long with regard to $L$. The orders of magnitude of $A, D$ and $L$ are not independent, but are all expressed by means of a single small parameter $\varepsilon$, through $A \propto \varepsilon^{2}, L \propto 1 / \varepsilon$ and $D \propto 1 / \varepsilon^{3}$, as shown in figure 1. Let us compare these assumptions to the historical soliton observed by Russell [1]. It was 'preserving its original figure some thirty feet long and a foot to a foot and a half in height', and was lost 'after a chase of one or two miles'. The amplitude $A \sim 0.3 \mathrm{~m}$ is small relative to both the channel depth and the length $L \sim 10 \mathrm{~m}$ of the solitary wave, and propagated on a distance $D$ of 2 or $3 \mathrm{~km}$. However, the amplitude is rather high with respect to a $\mathrm{KdV}$ approximation valid on such a distance. It would indeed involve a perturbation parameter $\varepsilon \sim \sqrt{L / D} \sim 1 / 10$, and the amplitude in the $\mathrm{KdV}$ approximation should be $A \sim \varepsilon^{3} L \sim 1 \mathrm{~cm}$. For much higher amplitude, higher-order corrections to the $\mathrm{KdV}$ model must be taken into account. However, they modify the soliton shape and speed, but do not prevent it from existing, as the experiment proves. Due to the robustness of solitons, the validity range of the perturbative computation is in general much wider than what is expected from the mathematics.

The relative size of the various terms is determined as follows. The $\mathrm{KdV}$ equation is

$$
\partial_{\tau} g+P g \partial_{\xi} g+Q \partial_{\xi}^{3} g=0 .
$$

It involves a spatial derivative $\partial_{\xi}^{3} g$, which accounts for dispersion. It can easily be proved that the first-order derivative $\partial_{\xi} g$ can account for propagation only and that the secondorder derivative $\partial_{\xi}^{2} g$ involves energy losses. This justifies that the dominant dispersion term has this form. If the length of the solitary wave is large, $L \sim 1 / \varepsilon$ with $\varepsilon \ll 1$, then the dispersion term is about $\partial_{\xi}^{3} g \sim \varepsilon^{3} g$. It must arise at the propagation distance (or equivalently propagation time) $D$, accounted for in the equation by the term $\partial_{\tau} g \sim g / D$. Hence $1 / D \sim \varepsilon^{3}$. Regarding the nonlinear term, if it has the form $g \partial_{\xi} g$ its order of magnitude is $g^{2} / L \sim \varepsilon g^{2}$. The formation of the soliton requires that the nonlinear effect balance the dispersion. Hence it must have the same order of magnitude and $\varepsilon^{3} g=\varepsilon g^{2}$. Hence $g \sim \varepsilon^{2}$.

In principle, the model derived this way is valid for waves with amplitudes and lengths and for propagation distances which satisfy these conditions and for very small values of $\varepsilon$ only. Many experiments, such as the observation by Russell, show that the validity range of the model is in many cases much wider, at least for the qualitative behaviour of the wave.

\subsection{A model and scales}

In [32, 33] the derivation of a KdV-type asymptotic was studied, starting from a rather general class of systems of PDEs, which contains in particular the Maxwell-Landau model which is used in the description of electromagnetic wave propagation in a ferromagnetic dielectric (cf section 6.1). The general system can be written as

$$
\left(\partial_{t}+A \partial_{x}+E\right) \vec{u}=B(\vec{u}, \vec{u}),
$$

where the function $\vec{u}$ of the variables $x$ and $t$ is valued in $\mathbb{R}^{p}, A$ and $E$ are $p \times p$ matrices and $B: \mathbb{R}^{p} \times \mathbb{R}^{p} \longrightarrow \mathbb{R}^{p}$ is symmetrical bilinear $\left(\partial_{x}\right.$ denotes the partial derivative operator $\partial / \partial x)$. For the sake of clarity, we choose special matrices $A$ and $E$, that allow us to put in evidence the hypothesis that appears during the derivation of the general case, together with their interpretation from a more physical point of view, while reducing the computations and abstract notations as much as possible. These matrices are

$$
\begin{aligned}
A & =\left(\begin{array}{cccc}
1 & 0 & 2 & 1 \\
0 & -1 & 1 & 1 \\
2 & 1 & 0 & 0 \\
1 & 1 & 0 & 0
\end{array}\right), \quad E=\left(\begin{array}{llll}
0 & 0 & 0 & 0 \\
0 & 0 & 0 & 0 \\
0 & 0 & 0 & 1 \\
0 & 0 & s & 0
\end{array}\right) \\
\vec{u} & =\left(\begin{array}{l}
u \\
v \\
q \\
r
\end{array}\right)
\end{aligned}
$$

with $s$ being a real parameter. No special assumption is made about $B$. The vectors of the canonical basis will be denoted by $\vec{\alpha}, \vec{\beta}, \vec{\gamma}, \vec{\delta}$, and the corresponding components of $B$ by $B_{\alpha}, B_{\beta}, B_{\gamma}$ and $B_{\delta}$. The choice of $A$ and $E$ is motivated for the sake of simplicity in the presentation and does not correspond to any particular physical situation.

The function $\vec{u}$ is expanded in a power series of a small parameter $\varepsilon$ as

$$
\vec{u}=\varepsilon^{2} \vec{u}_{2}+\varepsilon^{3} \vec{u}_{3}+\cdots,
$$

where $\vec{u}_{2}, \vec{u}_{3}$, etc are functions of 'slow variables' defined by

$$
\xi=\varepsilon(x-V t), \quad \tau=\varepsilon^{3} t,
$$

with $V$ being a velocity to be determined. Let us check that equation (5) accounts for the scales shown in figure 1 . The wave profile will be described by some function $g(\xi)$. Its 
length corresponds thus to some fixed value $\xi_{0}$ of $\xi$. According to (5), it yields a length of the solitary wave in physical units $L=(x-V t)_{0}=\xi_{0} / \varepsilon$, large with order $1 / \varepsilon$. Analogously, the evolution is accounted for by values of the $\tau$ variable. A given propagation time corresponds to a finite value $\tau_{0}$ of $\tau$ and hence to a large time $t_{0}=\tau_{0} / \varepsilon^{3}$. The corresponding distance $D \sim 1 / \varepsilon^{3}$ is obtained by multiplying $t_{0}$ by the finite velocity $V$.

The fact that the expansion begins with a term of order $\varepsilon^{2}$ is equivalent, physically, to specifying the order of magnitude of the amplitude, according to figure 1. A priori, expansion (4) may begin with a term of order $\varepsilon$, but it is easily checked that, for the considered time scales, this does not yield any evolution equation.

At first order $\varepsilon^{2}$, equation (2) gives

$$
E \vec{u}_{2}=0 \text {, }
$$

that is $q_{2}=r_{2}=0$. At following order $\varepsilon^{3}$, we get the equation

$$
E \vec{u}_{3}+(A-V) \partial_{\xi} \vec{u}_{2}=0
$$

which yields, assuming that $\vec{u}$ vanishes at infinity and taking into account the results of the previous order,

$$
(1-V) u_{2}=0, \quad(-1-V) v_{2}=0,
$$

for the two first equations, in the kernel of $E$. It is seen that two propagation velocities are allowed: $V= \pm 1$. One of the first effects of the ansatz (5) is to select a single velocity. We choose arbitrarily $V=+1$. While $v_{2}=0, \vec{u}_{2}$ describes then a wave with some polarization, corresponding to this choice: $\vec{u}_{2}=g \vec{\alpha}$, where $g$ is a function of the slow variables to be determined. The choice of the special matrix $A$ assumes that $V$ is a simple eigenvalue. It is clear that, generally, this is a necessary condition to obtain the KdV asymptotic. On the other hand, it can be noticed that $V$ and $\vec{\alpha}$ are not the eigenvalue and eigenvector of $A$ itself, but of its restriction to the kernel of $E$ and projection onto it. The components of the system (7) outside the kernel of $E$ allow us to obtain the expressions of $q_{3}$ and $r_{3}$,

$$
q_{3}=-2 g_{\xi}, \quad r_{3}=-\frac{1}{s} g_{\xi},
$$

where the index denotes the derivative $\left(g_{\xi}=\partial_{\xi} g\right)$.

At order $\varepsilon^{4}$, the equation is

$$
E \vec{u}_{4}+(A-V) \partial_{\xi} \vec{u}_{3}=B\left(\vec{u}_{2}, \vec{u}_{2}\right) .
$$

It can be split into three parts: the $u$ component, corresponding to the selected propagation mode, the $v$ component, corresponding to the other (in the general case: others) propagation mode that belongs to the kernel of $E$, and the two others components that are not in this kernel. The $u$ component of equation (10) is a compatibility condition for $g$,

$$
-2(1+1 / s) g_{\xi \xi}=g^{2} B_{\alpha}(\vec{\alpha}, \vec{\alpha}) .
$$

If the coefficients of equation (11) are not both zero, it does not have any nonzero bounded solution. The following conditions must thus be satisfied (hypotheses 1 and 2):

$$
s=-1 \quad \text { and } \quad B_{\alpha}(\vec{\alpha}, \vec{\alpha})=0 .
$$

The equation corresponding to the $v$ component, or the $\vec{\beta}$ mode, gives

$$
v_{3}=\frac{-1}{2} g_{\xi}-\frac{1}{2} B_{\beta}(\vec{\alpha}, \vec{\alpha}) \int^{\xi} g^{2},
$$

while the last two equations give expressions for the components that do not belong to the kernel of $E$ :

$q_{4}=\varphi_{\xi}+\frac{3}{2} g_{\xi \xi}-\left(\frac{1}{2} B_{\beta}(\vec{\alpha}, \vec{\alpha})+B_{\delta}(\vec{\alpha}, \vec{\alpha})\right) g^{2}$,
$r_{4}=-2 \varphi_{\xi}+\frac{3}{2} g_{\xi \xi}+\left(\frac{1}{2} B_{\beta}(\vec{\alpha}, \vec{\alpha})+B_{\gamma}(\vec{\alpha}, \vec{\alpha})\right) g^{2}$,

where we have set $u_{3}=\varphi$.

Using the previous results, we get, from the compatibility conditions, i.e. from the $u$ component of the equation obtained at order $\varepsilon^{5}$, the evolution equation for $g$ as

$$
\partial_{\tau} g+P g \partial_{\xi} g+Q \partial_{\xi}^{3} g=R \varphi_{2} \int^{\xi} g^{2}
$$

The scalar coefficients $P, Q$ and $R$ are defined by

$$
\begin{aligned}
P= & -B_{\beta}(\vec{\alpha}, \vec{\alpha})+2 B_{\gamma}(\vec{\alpha}, \vec{\alpha})-4 B_{\delta}(\vec{\alpha}, \vec{\alpha})+B_{\alpha}(\vec{\alpha}, \vec{\beta}) \\
& -2 B_{\alpha}(\vec{\alpha}, \vec{\gamma})+4 B_{\alpha}(\vec{\alpha}, \vec{\delta}) \\
R= & -B_{\beta}(\vec{\alpha}, \vec{\alpha}) B_{\alpha}(\vec{\alpha}, \vec{\beta})
\end{aligned}
$$

and $Q=9 / 2$. If the additional condition $R=0$ is satisfied (hypothesis 3), equation (16) reduces to the $\mathrm{KdV}$ equation

$$
\partial_{\tau} g+P g \partial_{\xi} g+Q \partial_{\xi}^{3} g=0 .
$$

Let us recall that this equation is the first one that was solved by the IST method $[5,34]$ and that the first observed soliton, a wave in a canal, can be described by this model.

\subsection{Physical meaning of the hypotheses}

In addition to the hypotheses implicitly made by the particular choice of the matrices $A$ and $E$, especially the fact that the selected propagation mode is non-degenerated, the above formal derivation of the $\mathrm{KdV}$ equation necessitates three hypotheses that have been clarified. Two of them involve the nonlinearity, the third concerns in fact a derivative of the dispersion relation of the linearized system. Let us consider the latter. The linearized system is

$$
\left(\partial_{t}+A \partial_{x}+E\right) \vec{u}=0 .
$$

The pulsation and the polarization vector corresponding to a given wave vector $k$ will be denoted by $\omega(k)$ and $\vec{u}(k)$, respectively. They satisfy

$$
(-\mathrm{i} \omega(k)+A \mathrm{i} k+E) \vec{u}(k)=0 .
$$

The dispersion relation is drawn in figure 2. It contains some elements which are characteristic for a large number of physical dispersion relations and can be used as a typical example for their interpretation. To analyse it in the long-wave approximation, i.e. when $k$ tend to 0 , we look for $\omega$ in the form of a power series of $k$ as

$$
\omega=\omega_{0}+a k+b k^{2}+c k^{3}+O\left(k^{4}\right) .
$$

At order $k^{0}$, it is seen that $\omega_{0}$ must be an eigenvalue of $E$. In the considered special case, and taking $s=-1, E$ has two nonzero eigenvalues 1 and -1 , for the eigenvectors $\vec{\gamma}_{ \pm}=\vec{\gamma} \pm \mathrm{i} \vec{\delta}$. These values of the pulsation $\omega$ correspond to global oscillations of the material that do not propagate and that I will denote by resonances, cf figure 2. Zero is a double eigenvalue of $E$. Then, let us try to determine the 


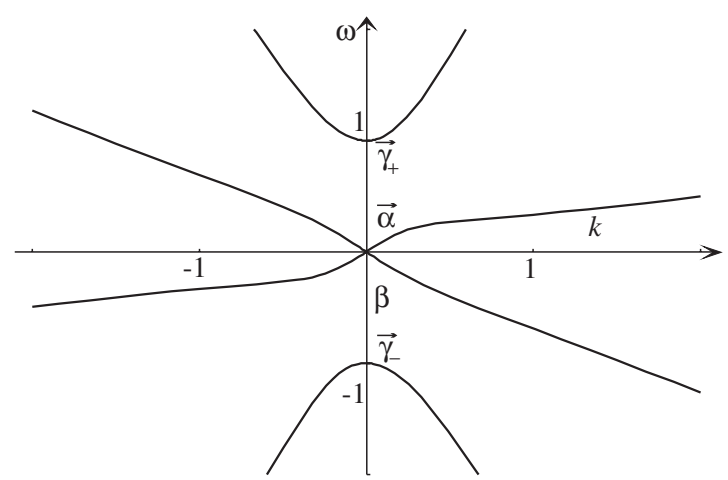

Figure 2. The dispersion relation of a linearized abstract model, showing the 'long-wave' type modes $\vec{\alpha}$ and $\vec{\beta}$, and the 'resonant' modes $\vec{\gamma}_{+}$and $\vec{\gamma}_{-}$.

following order in the $k$ expansion of the dispersion relation. It is found that $a= \pm 1$, with either $u$ or $v$ null, i.e. two modes, with the polarization vectors $\vec{\alpha}$ and $\vec{\beta}$ and the velocities $V=\omega / k= \pm 1$. We retrieve the two propagation modes of long-wave type obtained during the derivation of the $\mathrm{KdV}$ equation. We have been able to determine the role of the various components of the system, and in particular to give an interpretation of those belonging to the kernel of $E$ as resonances.

Continuing the computation to the following order, having chosen $a=+1$, i.e. the $\vec{\alpha}$ mode, we obtain the value of $b$, from which the second derivative of the dispersion relation is deduced: $\mathrm{d}^{2} \omega /\left.\mathrm{d} k^{2}\right|_{k=0}=-4 \mathrm{i}(1+1 / s)$. It is deduced that the condition $s=-1$, hypothesis 1 , is equivalent to

$$
\left.\frac{\mathrm{d}^{2} \omega}{\mathrm{d} k^{2}}\right|_{k=0}=0 .
$$

It is here equivalent to the requirement that $E$ is antisymmetric, which ensures that the system is conservative. Computing the coefficient $c$ in the same way, it is shown that the coefficient $Q$ of the $\mathrm{KdV}$ equation (19) is

$$
Q=\frac{-1}{3} \frac{\mathrm{d}^{3} \omega}{\mathrm{d} k^{3}}(0) .
$$

Formulae (23) and (24) are in fact general.

The two hypotheses, 2 and 3, that concern the nonlinearity, obviously cannot be explained by the study of the linearized equation (20). These are particular expressions of a very general condition called the 'transparency' condition in the rigorous mathematical theory of multiscale expansions by Joly, Métivier and Rauch [30]. Hypothesis 2 excludes the quadratic self-interaction of the chosen propagation mode, while hypothesis 3 excludes the interaction of distinct polarizations at same order. If hypothesis 2 is not satisfied, the nonlinear term appears sooner in the expansion. The nonlinear evolution of the wave will be described by a nonlinear evolution equation other than $\mathrm{KdV}$, and for other space, amplitude and time scales. If the hypothesis 3 is not satisfied, the above computation is valid, but the evolution equation is equation (16) instead of $\mathrm{KdV}$. It is an integro-differential equation, involving a cubic nonlinear term.

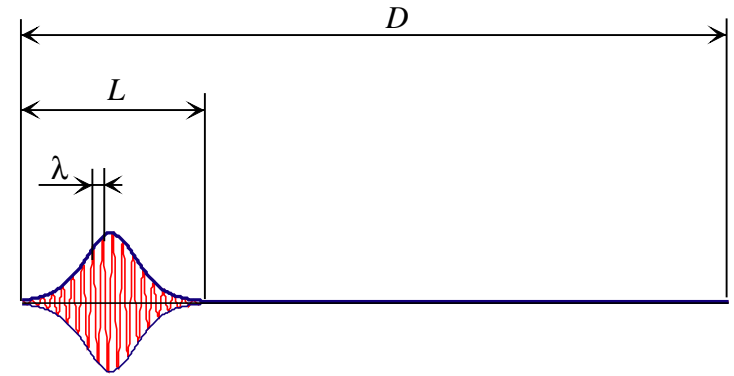

Figure 3. The various length scales of an envelope problem. $\lambda$ : wavelength, $L$ : pulse length, large with respect to $\lambda, D$ : propagation distance, large with respect to $L$.

\section{Envelopes}

\subsection{The model for envelope solitons}

The nonlinear evolution of a wave packet involves several scales, which are either lengths measured along the propagation direction or the corresponding time, the phase velocity being a constant of the problem [6]. The first length scale is the wavelength $\lambda$ of the carrier that will be the reference length (zero order). Intervening then is the length $L$ of the wave packet, which must be large with regard to the wavelength, so that an approximation of slowly varying envelope type can be envisaged. A third scale is the propagation distance $D$, cf figure 3. The evolution of the envelope on distances $D$ of the same order as $L$ - there would be in this case two scales onlyis, in the linear case, a transport at the group velocity. The effect of the dispersion appears at larger propagation distances. The situation where a nonlinear effect perturbs this transport has been introduced by Hunter under the name of nonlinear geometrical optics [21]. It has given rise to the first rigorous convergence proofs, cf section 1.4. What we are interested in here is the formation of solitons that represent, according to certain definitions, an equilibrium between the dispersion and the nonlinear effect. The propagation distances $D$ we have to consider are thus those at which the dispersion appears. $D$ will thus typically have the same order of magnitude as the dispersion length or temporal Fresnel length

$$
L_{\mathrm{f}}=\frac{\Delta t^{2}}{2 \pi k_{2}} .
$$

In this expression, $k_{2}=\mathrm{d}^{2} \omega / \mathrm{d} k^{2}$ measures the dispersion and $\Delta t$ is the initial pulse duration. With the above notations, $\Delta t=n L / c$ and $k_{2}=\left(2 n^{\prime}+\omega n^{\prime \prime}\right) / c$, where $n^{\prime}$ and $n^{\prime \prime}$ are the first and second derivatives of the refraction index $n$ with regard to the pulsation $\omega=2 \pi c / \lambda$. Hence, from expression (25) we get

$$
D=L_{\mathrm{f}}=\frac{n^{2}}{2 n^{\prime} \omega+n^{\prime \prime} \omega^{2}} \frac{L^{2}}{\lambda} .
$$

It is easily seen that the ratio $n^{2} /\left(2 n^{\prime} \omega+n^{\prime \prime} \omega^{2}\right)$ is dimensionless. The most simple procedure is to treat it as a number of order $\varepsilon^{0}$. From this, it is deduced that

$$
\frac{\lambda}{L} \sim \frac{L}{D} \sim \varepsilon,
$$

which will be the perturbative parameter. 
Let us consider as a numerical example the first solitons created in an optical fibre by Mollenauer et al [10]. The wavelength was $\lambda=1.55 \mu \mathrm{m}$, the propagation distance, i.e. the length of the fibre, $D=700 \mathrm{~m}$, and the pulse duration $\Delta t=7 \mathrm{ps}$, which corresponds to a length $L=1.4 \mathrm{~mm}$. We get $\lambda / L=10^{-3}$ and $L / D=2 \times 10^{-6}$. A first rough approach is to take, e.g., $\varepsilon=2 \times 10^{-5}$, and then claim that $\lambda / L=50 \varepsilon$ and $L / D=0.1 \varepsilon$ have the same order of magnitude as $\varepsilon$. It is correct in some sense; in any case it works. However, we can do better if we take into account the fact that the dispersion coefficient $\left(2 n^{\prime} \omega+n^{\prime \prime} \omega^{2}\right) / n^{2}$ is small in a silica fibre at the considered wavelength. Using the data of [10] we get a value about $7 \times 10^{-3}$ for this quantity, and it is seen that relation (26) holds with quite good accuracy. For technical reasons, it is quite difficult to include the assumption that the dispersion coefficient is small in the multiscale expansion. In general the first approach will be used for the mathematical derivation and the second to specify the validity range of the result in some specific physical situation.

The nonlinear effects will appear at a propagation distance with such an order of magnitude if the wave amplitude belongs to a given range. Formally, a weak amplitude of order $\varepsilon$ is considered. This must obviously be understood with regard to some reference amplitude that, physically, depends on coupling constants. Due to dimension problems, to fix the reference amplitude is not obvious. However, if the order zero is assigned to the physical value of the coupling constant, the order of magnitude of the amplitude is that of the ratio of the second harmonic (produced in a non-resonant way) to the fundamental. More precisely, it is assumed that all the coupling constants have the same order of magnitude. Then the reference amplitude $A_{0}$ is such that the order of magnitude of the $n$th harmonic (produced in a non-resonant way) is $\left(A_{0}\right)^{n}$. Except in a few very specific situations, this simplifying assumption is consistent with experimental results. Otherwise the weakest couplings might be disregarded. A further assumption can also be included in the perturbative scheme to account for their weakness, in a way similar to the weak damping assumption introduced in section 6.2. The above considerations give a physical interpretation of the weak amplitude hypothesis, valid in any physical frame and more especially in nonlinear optics. In other physical frames, the wave amplitude can easily be compared to another quantity involved in the problem, with the same dimension, that will be used as a reference. As an example, concerning the hydrodynamical surface waves, the wave amplitude is a length and can thus be compared to another characteristic length of the problem. In magnetic media, either the saturation magnetization of the material or an applied magnetic field will be used as a reference.

Let us consider the set of equations

$$
\begin{gathered}
u_{x x}-u_{t t}=u+\beta(u+v)^{2}, \\
v_{x x}-v_{t t}=-v-\beta(u-v)^{2} .
\end{gathered}
$$

Equations (28) and (29) have no physical meaning, but allow us to present the method with a minimum of computations, avoiding the introduction of abstract notations. We look for solutions of the form

$$
\vec{u}=\vec{u}_{1}\left(\varepsilon(x-V t), \varepsilon^{2} t\right) \mathrm{e}^{\mathrm{i}(k x-\omega t)}+\text { c.c. }+O\left(\varepsilon^{2}\right)
$$

$(\vec{u}=(u, v)$, c.c.: complex conjugate). $\vec{u}$ is expanded simultaneously in a power series of the perturbative parameter $\varepsilon$ and in a Fourier series of the fundamental phase $\varphi=k x-\omega t$ as

$$
\vec{u}=\sum_{n \leqslant 1, p} \varepsilon^{n} \vec{u}_{n}^{p} \mathrm{e}^{\mathrm{i} p \varphi} .
$$

In this expansion, the profiles $\vec{u}_{n}^{p}=\left(u_{n}^{p}, v_{n}^{p}\right)$ are functions of slow variables defined by

$$
\xi=\varepsilon x, \quad T=\varepsilon t, \quad \tau=\varepsilon^{2} t .
$$

The variable $\tau$ accounts for the evolution on long-range propagation. Indeed, a given value $\tau_{0}$ of $\tau$ corresponds to a time $t=\tau_{0} / \varepsilon^{2}$ and to a propagation distance $D=V \tau_{0} / \varepsilon^{2}$. The shape of the pulse is accounted for by the variable $\xi$; a given value $\xi_{0}$ of $\xi$ corresponds in the same way to a length $L=\xi_{0} / \varepsilon$. Since the wavelength $\lambda$ is assumed to be the reference length of order $\varepsilon^{0}$ by the above definitions, it is seen that the relation (27) is satisfied. The variable $T$ of the same order as $\xi$ accounts for the evolution of the wave on propagation distances comparable to the pulse length. It will be seen that this evolution is the transport at the group velocity, $V$, accounted for by a mere $(x-V t)$ dependency. I do not introduce a priori this dependency, in order to show how it appears by itself during the application of the perturbation method. Regrouping the terms in $\varepsilon^{1} \mathrm{e}^{\mathrm{i} p \varphi}$, we obtain the equations

$$
\begin{gathered}
\left(-p^{2} k^{2}+p^{2} \omega^{2}\right) u_{1}^{p}=u_{1}^{p} \\
\left(-p^{2} k^{2}+p^{2} \omega^{2}\right) v_{1}^{p}=-v_{1}^{p}
\end{gathered}
$$

There are two possible polarizations, $u$ or $v$, and for each one a dispersion relation:

$$
p^{2} \omega^{2}=p^{2} k^{2} \pm 1
$$

The expansion (31) assumes that $\omega$ and $k$ each have a unique value. This implies that a polarization and a particular branch of the dispersion relation (35) must be chosen and furthermore that the latter would be satisfied by a single harmonic, arbitrarily chosen. Physically, this means that we exclude taking into account the multiple wave interactions, such as stimulated diffusions or resonant harmonic generation. The study of these necessitates a modification of the ansatz, an example of which will be given in the following section. Note that in some particular situations a formal expansion will be able to yield an asymptotic model, which leaves the diffusions aside, but whose convergence will be prevented by them. We choose

$$
u_{1}^{1}=f(\xi, T, \tau), \quad \omega=\sqrt{k^{2}+1},
$$

and all other components $u_{1}^{p}, v_{1}^{p}$ are zero (except $u_{1}^{-1}=f^{*}$ ). At order $\varepsilon^{2}$, we obtain the equations

$$
\begin{aligned}
& \left(-p^{2} k^{2}+p^{2} \omega^{2}\right) u_{2}^{p}+2 \mathrm{i} p\left(k \partial_{\xi}+\omega \partial_{T}\right) u_{1}^{p}=u_{2}^{p}+R_{2}^{p} \\
& \left(-p^{2} k^{2}+p^{2} \omega^{2}\right) v_{1}^{p}+2 \mathrm{i} p\left(k \partial_{\xi}+\omega \partial_{T}\right) u_{1}^{p}=-v_{1}^{p}+S_{2}^{p},
\end{aligned}
$$


where $R_{2}^{p}$ and $S_{2}^{p}$ are nonlinear terms defined by

$$
\begin{gathered}
R_{2}^{p}=\beta \sum_{q+r=p}\left(u_{1}^{q}+v_{1}^{q}\right)\left(u_{1}^{r}+v_{1}^{r}\right), \\
S_{2}^{p}=-\beta \sum_{q+r=p}\left(u_{1}^{q}-v_{1}^{q}\right)\left(u_{1}^{r}-v_{1}^{r}\right) .
\end{gathered}
$$

The evolution of the amplitude $f$ at the first order, i.e. for times $t$ about $1 / \varepsilon$ for which $T=\varepsilon t$ has a finite value, is given by equation (37) for the fundamental harmonic $p=1$. The nonlinear term $R_{2}^{1}$ is zero. The terms involving $u_{2}^{1}$ simplify, due to the dispersion relation (36), and we get finally

$$
\left(k \partial_{\xi}+\omega \partial_{T}\right) f=0 .
$$

The solution of this transport equation is obviously $f=$ $f(\xi-V T)$, with $V=k / \omega$. We check that the expression of $V$ coincides with the usual expression of the group velocity $V=\mathrm{d} \omega / \mathrm{d} k$, using the dispersion relation (36).

We note that $u_{2}^{1}$ is free, together with $u_{2}^{-1}=u_{2}^{1^{*}}$, and that the other profiles $u_{2}^{p}$ and $v_{2}^{p}$ are entirely determined: this is imposed by the choice of the polarization of the leading order. The nonzero nonlinear terms are $R_{2}^{ \pm 2}=-S_{2}^{ \pm 2}=\beta f^{2}$ and $R_{2}^{0}=2-S_{2}^{0}=\beta|f|^{2}$ only. The nonzero terms of the second order are deduced from this; they are

$u_{2}^{0}=v_{2}^{0}=-2 \beta|f|^{2}, \quad u_{2}^{2}=\frac{\beta}{3} f^{2}, \quad v_{2}^{2}=\frac{-\beta}{5} f^{2}$,

and their complex conjugates.

The evolution of the amplitude $f$ at second order, i.e. for times $t$ of order $1 / \varepsilon^{2}$, for which the slow variable $\tau$ has a finite value, is given by equation (28), at order $\varepsilon^{3}$, and for the fundamental harmonic $p=1$. It is written, after simplification of the terms in $u_{3}^{1}$,

$$
\begin{aligned}
& 2 \mathrm{i}\left(k \partial_{\xi}+\omega \partial_{T}\right) u_{2}^{1}+\partial_{\xi}^{2} f+2 \mathrm{i} \omega \partial_{\tau} f+\partial_{T}^{2} f \\
& =2 \beta\left[f\left(u_{2}^{0}+v_{2}^{0}\right)+f^{*}\left(u_{2}^{2}+v_{2}^{2}\right)\right] .
\end{aligned}
$$

The key of the problem is the determination of the evolution of the correction at second order $u_{2}^{1}$ with regard to the first-order time $T$. Let us consider it in detail. We make the change of variables

$$
\tilde{\xi}=\xi-V T, \quad \tilde{T}=T .
$$

Equation (43) can then be put into the form

$$
\partial_{\tilde{T}} u_{2}^{1}=\Phi(\tilde{\xi}) .
$$

Indeed, $f$ is a function of $\tilde{\xi}$ only thus, according to (42), the same holds for $u_{2}^{0}, v_{2}^{0}, u_{2}^{2}$ and $v_{2}^{2}$. Hence,

$$
u_{2}^{1}=\tilde{T} \Phi(\tilde{\xi})+u_{2}^{1}(0) \text {. }
$$

If $\tilde{T}$ (or $T$ ) remained bounded, we could stop here, but we are looking for the evolution of $f$ at times $t$ of the order of $1 / \varepsilon^{2}$, i.e. for values of $\tilde{T}$ of the order of $1 / \varepsilon$. For these values, $u_{2}^{1}$ given by (46) is no longer a term of order $\varepsilon^{2}$, but becomes of order $\varepsilon$, comparable to the leading term $u_{1}^{1}=f$. It is consistent to incorporate this 'secular' part of $u_{2}^{1}$ into the leading term $f$. Hence we impose that the solution $u_{2}^{1}$ remains bounded or more exactly sublinear: $\lim _{T \rightarrow+\infty} u_{2}^{1} / T=0$. From this we deduce that $u_{2}^{1}$ is a function of $\tilde{\xi}=\xi-V T$ only and that $\Phi(\tilde{\xi})=0$, i.e.

$$
\partial_{\xi}^{2} f+2 \mathrm{i} \omega \partial_{\tau} f+\partial_{T}^{2} f=-2 \beta\left(f u_{2}^{0}+f^{*} u_{2}^{2}\right) .
$$

Using (41) and (42), equation (47) reduces to the nonlinear Schrödinger equation (NLS)

$$
2 \mathrm{i} \omega \partial_{\tau} f+\left(1-\frac{k^{2}}{\omega^{2}}\right) \partial_{\xi}^{2} f+\frac{116}{15} \beta^{2} f|f|^{2}=0 .
$$

We check directly from the dispersion relation (36) that the coefficient of the dispersive term is

$$
\left(1-\frac{k^{2}}{\omega^{2}}\right)=\omega \frac{\mathrm{d}^{2} \omega}{\mathrm{d} k^{2}}
$$

The nonlinear term of the initial system (28), (29) has been chosen in order to give an example of all nonlinear contributions that may appear in the term of self-phase modulation for a quadratic nonlinearity. These terms always involve some intermediary, that is either the second harmonic $\left(u_{2}^{2}\right.$ in the example) or the rectified or mean value field $u_{2}^{0}$. In the example, the nonlinear dependency with regard to the second field component $v$ was chosen in such a way that both the cascaded interactions through $v_{2}^{2}$ and the one through $v_{2}^{0}$ are involved by the nonlinear coefficient of the NLS equation. Hence it can be noticed that, even if a single polarization component, here $u$, is involved by the first order, the harmonics of the other polarization can intervene in the expression of the self-phase modulation coefficient. Of course, in a given situation, this depends on the physical properties of the nonlinear interactions between the various polarization components.

\subsection{Interactions of wave packets}

To provide evidence the interactions of envelopes, we use a similar procedure; the difference is that several fundamental phases are considered. Several interaction types can be described this way: resonant or non-resonant, and involving more or fewer waves. The description of the non-resonant interactions remains in principle very close to the previous case. The resonant interactions present interesting features, in particular the fact that two scales only are involved. Indeed, the resonant interaction may occur on propagation distances of the order of the pulse size, which reduces the number of scales to two. Further, the pulses that interact this way have in general group velocities that differ from each other. They thus cannot coexist on propagation distances that are large with regard to their length. Their dispersion will thus be negligible during the interaction. The typical case is that of three waves, for which the asymptotic model is completely integrable: this is the situation we are interested in.

Le us consider the same academic example (28), (29) as in the previous subsection. The expansion will now have the form

$$
\vec{u}=\sum_{j=1,2,3} \sum_{n \leqslant 1, p} \varepsilon^{n} \vec{u}_{j, n}^{p} \mathrm{e}^{\mathrm{i} p \varphi_{j}},
$$

in which $\varphi_{j}=k_{j} x-\omega_{j} t$, for $j=1,2,3$, are three fundamental phases, and the profiles $\vec{u}_{j, n}^{p}=\left(u_{j, n}^{p}, v_{j, n}^{p}\right)$ are functions of the slow variables:

$$
\xi=\varepsilon x, \quad T=\varepsilon t .
$$


Note the change with regard to the previous section: there is no 'long' propagation variable $\tau$ here, for the reasons mentioned above. We assume that the leading term is

$$
\vec{u}=\varepsilon\left(\vec{u}_{1} \mathrm{e}^{\mathrm{i} \varphi_{1}}+\vec{u}_{2} \mathrm{e}^{\mathrm{i} \varphi_{2}}+\vec{u}_{3} \mathrm{e}^{\mathrm{i} \varphi_{3}}+\text { c.c. }\right)+O\left(\varepsilon^{2}\right),
$$

where we have set $u_{j, 1}^{1}=u_{j}(j=1,2,3)$. For convenience, we also use the notations $u_{-j}=u_{j, 1}^{-1}$ and $\varphi_{-j}=-\varphi_{j}$, for $j=1,2,3$, so we can write equation (52) in the form $\vec{u}=\varepsilon \sum_{j= \pm 1, \pm 2, \pm 3} \vec{u}_{j} \mathrm{e}^{\mathrm{i} \varphi_{j}}+O\left(\varepsilon^{2}\right)$.

To the first order in $\varepsilon$, it is found that $u_{j}$ must satisfy equations (33) and (34), i.e. that each wave belongs to some linear mode. We assume that waves 1 and 2 belong to the $u$ mode and wave 3 to the $v$ mode, i.e.

$\omega_{j}=\sqrt{k_{j}^{2}+1}, \quad \vec{u}_{j}=\left(u_{j}, 0\right), \quad$ for $\quad j=1,2$,

$\omega_{3}=\sqrt{k_{3}^{2}-1}, \quad \vec{u}_{3}=\left(0, v_{3}\right)$.

At order $\varepsilon^{2}$, for the $u$ component and the harmonic $\mathrm{e}^{\mathrm{i} \varphi_{j}}$, we get the equation

$$
\begin{aligned}
& \left(-k_{j}^{2} u_{j, 2}^{1}+2 \mathrm{i} k_{j} \partial_{\xi} u_{j}\right)-\left(-\omega_{j}^{2} u_{j, 2}^{1}-2 \mathrm{i} \omega_{j} \partial_{T} u_{j}\right) \\
& =u_{j, 2}^{1}+\beta \sum_{\varphi_{l}+\varphi_{m}=\varphi_{j}}\left(u_{l}+v_{l}\right)\left(u_{m}+v_{m}\right) .
\end{aligned}
$$

For wave 1, equation (55) becomes, taking (53) into account, and after division by $2 \mathrm{i} \omega_{1}$,

$$
\partial_{\xi} V_{1} u_{1}+\partial_{T} u_{1}=\frac{\beta}{\mathrm{i} \omega_{1}} \sum_{\varphi_{l}+\varphi_{m}=\varphi_{1}}\left(u_{l}+v_{l}\right)\left(u_{m}+v_{m}\right),
$$

where $V_{1}=k_{1} / \omega_{1}$ is the group velocity of wave 1 . If no resonance condition of the type $\varphi_{l}+\varphi_{m}=\varphi_{1}$ is realized, equation (56) reduces to

$$
V_{1} \partial_{\xi} u_{1}+\partial_{T} u_{1}=0
$$

which accounts for a mere transport at the group velocity. We assume thus that one of these resonance conditions is realized. By an adequate choice of the notations, it can always be written as $\varphi_{1}=\varphi_{2}+\varphi_{3}$, i.e.

$$
\omega_{1}=\omega_{2}+\omega_{3}, \quad k_{1}=k_{2}+k_{3} .
$$

Condition (58) accounts for the phase matching and can also be seen, if these are electromagnetic waves, as the condition for the conservation of the photon energy and impulsion. Equation (55) then becomes

$$
V_{1} \partial_{\xi} u_{1}+\partial_{T} u_{1}=\frac{\beta}{\mathrm{i} \omega_{1}} u_{2} v_{3} .
$$

For wave 2 , we have $\varphi_{2}=\varphi_{1}-\varphi_{3}$, thus the components involved by the nonlinear term of equation (55) are $\vec{u}_{1}=$ $\left(u_{1}, 0\right)$ and $\vec{u}_{-3}=\left(0, v_{3}^{*}\right)$. Equation (55) reduces to

$$
V_{2} \partial_{\xi} u_{2}+\partial_{T} u_{2}=\frac{\beta}{\mathrm{i} \omega_{2}} u_{1} v_{3}^{*} .
$$

Since wave 3 belongs to the other mode, the linear part of equation (55) for $j=3$ does not contain $f_{3}$, but contains $u_{3,2}^{1}$, and can be solved for the latter, whose value has no interest for us. In an analogous way, the other equation of the initial system, written at order $\varepsilon^{2}$ and for the Fourier component $\mathrm{e}^{\mathrm{i} \varphi_{3}}$, reduces to

$$
V_{3} \partial_{\xi} v_{3}+\partial_{T} v_{3}=\frac{-\beta}{\mathrm{i} \omega_{3}} u_{1} u_{2}^{*}
$$

The system yielded by the three equations (59), (60) and (61) is known under the name of 'three-wave resonant interaction system' (3WRI) [35]. It is completely integrable by the IST method [38].

\subsection{Stimulated backscattering and soliton exchange}

The transform $u_{1}=\mathrm{i} g_{1}, u_{2}=\mathrm{i} g_{2}, v_{3}=\mathrm{i} g_{3}$ allows us to write the 3 WRI system (59)-(61) in the standard form

$$
\begin{aligned}
& \partial_{T} g_{1}+V_{1} \partial_{\xi} g_{1}=A_{1} g_{2} g_{3}, \\
& \partial_{T} g_{2}+V_{2} \partial_{\xi} g_{2}=A_{2} g_{1} g_{3}^{*}, \\
& \partial_{T} g_{3}+V_{3} \partial_{\xi} g_{3}=A_{3} g_{1} g_{2}^{*},
\end{aligned}
$$

where $A_{1}=\beta / \omega_{1}, A_{2}=-\beta / \omega_{2}, A_{3}=\beta / \omega_{3}$.

In a special physical situation, the main question will be to determine whether the phase matching condition (58) can be realized, and how, taking into account the dispersion relation. In the case of the present example, with the above choice of the polarizations of the three waves, a solution exists for each $k_{2}$ and can be easily computed explicitly.

An important remark is that, in the frame of the reductive perturbation method, the phase matching is assumed to be realized exactly. It is well known in nonlinear optics, for cascaded second-order nonlinearities [39], that a phase mismatch does not prevent the interaction, if it is weak enough. The expression for wave 1 is

$$
\tilde{u}_{1}=\varepsilon f_{1}(\varepsilon x, \varepsilon t) \mathrm{e}^{\mathrm{i}\left(k_{1} x-\omega_{1} t\right)}+O\left(\varepsilon^{2}\right)
$$

Introducing a weak phase mismatch $k_{1}=k_{2}+k_{3}+\varepsilon \delta k=$ $k_{1}^{\prime}+\varepsilon \delta k$, it becomes

$$
\tilde{u}_{1}=\varepsilon f_{1}(\varepsilon x, \varepsilon t) \mathrm{e}^{\mathrm{i} \varepsilon \delta k x} \mathrm{e}^{\mathrm{i}\left(k_{1}^{\prime} x-\omega_{1} t\right)}+O\left(\varepsilon^{2}\right) .
$$

Setting $f_{1}^{\prime}(\varepsilon x, \varepsilon t)=f_{1}(\varepsilon x, \varepsilon t) \exp (\mathrm{i} \varepsilon \delta k x)$, the expression (64) of $\tilde{u}_{1}$ is reduced to (63), but with $k_{1}^{\prime}=k_{2}+k_{3}$, that is the exact phase matching. In other words, because the approach is perturbative, a zero represents more often an infinitely small quantity of order $\varepsilon$ than a quantity effectively equal to zero. Thus, the exact phase matching means approximate phase matching at order $\varepsilon$, due to the perturbative nature of the approach itself. However this gives rise to difficulties when one intends to apply it to a special physical situation. Indeed, mathematically, the perturbative parameter $\varepsilon$ is a varying quantity that can take values as small as we want. The phase mismatch $\delta k$, in general, does not depend on $\varepsilon$; it is finite, and thus, from the mathematical point of view, it is very large with regard to $\varepsilon$. But, in a concrete physical situation, the perturbative parameter $\varepsilon$ is a quantity that, although small, is fixed, and hence finite too, and it may happen that $\delta k$ is smaller that this finite value attributed to $\varepsilon$. In this case, the absence of nonlinear coupling which is deduced from the theory will contradict the experimental results. A procedure allowing us to describe the observed coupling, in a way that is satisfactory from the mathematical point of view, consists 

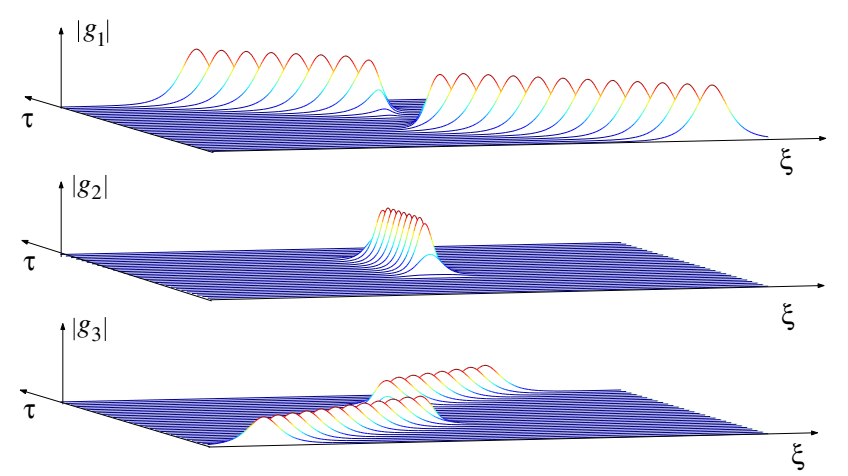

Figure 4. Resonant three-wave interaction: an incident optical soliton and an incident polariton soliton, almost resonant (top and bottom, in front), give rise to an optical soliton with different helicity (centre), which is transformed into two solitons again (top and bottom, rear). The chosen reference frame is bound to the wave with medium velocity.

of introducing a dependency of the dispersion relations with regard to the perturbative parameter, in such a way that the phase mismatch goes to 0 when $\varepsilon$ tends to 0 . Such an approach has been successfully applied to the nonlinear Faraday effect [40], parametric optical solitons [41, 42], and polarization interactions in a planar optical waveguide [43].

The 3WRI system (62) has been derived in the frame of electromagnetic waves in ferromagnetic media in [44]. Explicit expressions for the group velocities $V_{j}$ and the (real) interaction constants $A_{j}$ were obtained, allowing, together with the known results about the 3WRI system [35], identification of the various possible types of interaction.

We know three types of behaviour, depending on the relative signs $\delta_{j}=\operatorname{sgn} A_{j}$ of the interaction constants and on the relative magnitudes of the velocities. We assume that $V_{1}<V_{2}<V_{3}$. The three cases are as follows.

(i) The three constants have the same sign: $\delta_{1}=\delta_{2}=\delta_{3}$.

(ii) The constant whose sign differs from the others corresponds to the wave with the medium velocity: $\delta_{1}=$ $\delta_{3}=-\delta_{2}$.

(iii) The constant whose sign differs from the others corresponds to a wave with an extreme velocity, e.g., $\delta_{3}=-\delta_{2}=-\delta_{1}$.

The last case corresponds to a backscattering of wave 3 to wave 1 , stimulated by wave 2 (stimulated backscattering, $\mathrm{SBS}$ ). In ferromagnetic media, the typical situation is when waves 1 and 3 are two optical waves (branches $P O$ and $N$, see figure 12 in section 6.1) with opposite directions, and wave 2 is a magnetostatic wave (branch $P A$ ), propagating in the same direction as the incident wave 3 . The two optical waves can have either polarization, and thus the interaction can modify the wave polarization or not.

The interaction type (ii) is a soliton exchange. Each soliton contained in wave 2 with median velocity disappears to give rise to a soliton in each of the other two waves. Conversely, if the two waves 1 and 3 with extreme velocities contain two resonant solitons, the energy can be transferred to a soliton of the wave 2. The latter is however unstable: if the resonance is only approximate, the soliton of the wave 2 gives rise to the two initial solitons again. The soliton 3 , the faster one, is delayed, while the soliton 1, slower, is brought forward. This interaction can be described by explicit solutions [35], an example of which is drawn in figure 4. In the case of initial wave packets containing several solitons, each of which is in resonance with a soliton contained in the other wave, the main effect of the interaction is to quickly separate these solitonic components and to transform the incident wave packets into strings of solitons. In ferromagnetic media, this type of interaction can occur in particular between two 'optical' waves with different helicity and a polariton. Soliton exchange occurs when the polariton belongs to the 'acoustic' branch $P A$ and propagates in the same direction as the two other waves. When it propagates in the opposite direction, we can see evidence of a transition point: if the angle $\varphi$ between the propagation direction and the static field is between about $55^{\circ}$ and $90^{\circ}$, soliton exchange occurs, else stimulated backscattering occurs.

The interaction type (i) can lead to an explosion in a finite time. It does not occur in ferromagnetic media, at least for parallel plane waves. Dark solitons of the 3WRI system, and their interaction with bright solitons, have also been evidenced $[36,37]$.

\section{Multi-dimensional problems}

The models presented in the above sections are one dimensional. We chose for the presentation of the general NLS-type expansion the situation called 'temporal', where the variable that describes the shape of the wave packet is parallel to the propagation direction, to make use of the terminology of nonlinear optics. A continuous beam can also be considered, and its extension in a transverse dimension can be studied: it is the situation known as a 'spatial soliton'. Some essential properties of the reductive perturbation method appear only when the temporal dependency is considered. This justifies our presentation choice, since the 'spatial solitons' have no less interest and importance for physics than the 'temporal' solitons. On the other hand, several authors have already noticed that a laser pulse has always a finite duration and that the pulse called 'spatial' is always in fact spatio-temporal. This leads naturally to the study of bi- or tridimensional pulses.

\subsection{A 'spherical' pulse}

If in the multiscale expansion (31), (32), that leads to the NLS equation, $x$ is replaced by a tridimensional variable $\vec{x}$, the scales that will give an account of the wave packet evolution will be typically

$\xi=\varepsilon(x-V t), \quad \eta=\varepsilon y, \quad \zeta=\varepsilon z, \quad \tau=\varepsilon^{2} t$.

The change of scales defined by (65) can be used in the description of a 'spherical' pulse or, more exactly, of a pulse whose extension in the two transverse directions $y$ and $z$ and in the longitudinal direction $x$ has the same order of magnitude. In nonlinear optics, these kinds of pulses are called 'light bullets', when they propagate without deformation. 
Let us denote by $\xi_{j}$ one of the three slow variables $\xi, \eta$ and $\zeta$ that describe the shape of the wave packet. Taking into account the coherence constraint of the perturbation method, which imposes that integer powers of $\varepsilon$ only arise, what are the possible choices for $\xi_{j}$, apart from the one given by (65)? If $\xi_{j}$ is of order $\varepsilon^{0}$, the extension of the pulse in the corresponding direction will be of the same order as the wavelength $\lambda$. Then the slowly varying envelope approximation will no longer be valid. If in contrast $\xi_{j}$ is of order $\varepsilon^{2}$, the second derivative $\partial_{\xi_{j}}^{2}$ will not appear in the multi-dimensional evolution equation of NLS type that will be obtained. Thus, the diffraction or the dispersion corresponding to this variable will not be taken into account. In other words, all happens as if the pulse were infinite in this direction. This can be interpreted in a more physical way, for an optical 'spatial soliton': it is equivalent to considering the propagation of a beam on a distance small with regard to its diffraction length. The pulse does not propagate far enough to be diffracted. In its theoretical description, it can thus be considered as infinitely wide. Still regarding optical solitons, but in the temporal case, the situation is formally analogous. Let us consider some orders of magnitude. In numerous experimental situations, the propagation length $D$ is about a few $\mathrm{cm}$. Let us choose to fix $D=4 \mathrm{~cm}$. For a wavelength $\lambda=1 \mu \mathrm{m}$, the perturbative parameter is then $\varepsilon=\sqrt{\lambda / D}=200$. The characteristic length of the pulse, when it is described by a variable $\xi$ of order $\varepsilon^{1}, \xi=\varepsilon(x-V t)$, is $L=\varepsilon \lambda$, and the corresponding duration is $T=L / c \simeq 660$ fs. If the variable $\xi$ is in contrast of order $\varepsilon^{2}, \xi=\varepsilon^{2}(x-V t)$, the duration of the pulse becomes $T=\varepsilon^{2} \lambda / c=D / c \simeq 130$ ps. Thus, a pulse of 130 ps must be considered as infinitely long. Therefore, the change of scales (65), and the notion of the light bullet, can be meaningful for such a propagation distance, only if the pulse duration is at most about 1 ps. For some applications, the considered sizes are even smaller. The pulse duration for which the dispersion must be taken into account decreases in proportion.

However, even when the scale of the initial longitudinal pulse variations is long enough for the dispersion to be negligible, in the frame of a linear evolution, it may happen that the modulational instability or the stimulated diffusions create during the propagation a much faster longitudinal modulation, for which the dispersion is no longer negligible.

\subsection{Generalization of the NLS equation}

The theory of envelope soliton propagation in $(1+1)$ dimensions is based on the NLS equation (48), i.e., modifying a little the notations,

$$
\mathrm{i} A f_{\tau}+B f_{\xi \xi}+C f|f|^{2}=0,
$$

where $f$ is the complex amplitude of the wave, $\tau$ is the propagation variable, $\xi$ is the variable that describes the pulse shape and $A, B$ and $C$ are real coefficients. Note that the variable $\xi$ can also describe the transverse profile of the pulse as its longitudinal profile. In the latter case, it is ordinarily written in optics as a time variable $t$. In the same way, the evolution variable $\tau$ can be transformed from a time into a distance $z$, simply multiplying by the velocity. The latter case corresponds to the experimental situations in nonlinear optics, in which the propagation distance (length of the fibre, distance between two optical devices) is straightforwardly identified, while the propagation time is too fast to be accessed directly.

A well-known but essential property of the NLS equation is that it is completely integrable by means of the IST method whatever the value of the coefficients $A, B$ and $C[6,9]$. It admits solutions of soliton type if $B C>0$ and solutions of dark-soliton type if $B C<0$. The most simple generalization of the NLS equation to $(2+1)$ dimensions is

$$
\mathrm{i} A f_{\tau}+B f_{\xi \xi}+C f_{\eta \eta}+D f|f|^{2}=0,
$$

with the same notations as above and $A, B, C$ and $D$ being real coefficients. Equation (67) is called the two-dimensional nonlinear Schrödinger equation (2D NLS). It accounts, e.g., for the propagation of a continuous optical beam in a bulk isotropic Kerr medium. But the 2D NLS equation is never integrable and does not admit any stable localized solution: the self-focusing leads mainly to collapse [45].

Let us present another generalization of the NLS equation, which is 'better' in the sense that it conserves a large number of its properties, the Davey-Stewartson (DS) system:

$$
\begin{aligned}
& \mathrm{i} A f_{\tau}+B f_{\xi \xi}+C f_{\eta \eta}+D f|f|^{2}+E f \psi=0, \\
& \alpha \psi_{\xi \xi}+\beta \psi_{\eta \eta}=\gamma|f|_{\xi \xi}^{2}+\delta|f|_{\eta \eta}^{2} .
\end{aligned}
$$

The function $\psi$ is real valued, and $A, B, C, D, E, \alpha, \beta, \gamma$ and $\delta$ are real coefficients, which depend on the considered particular physical situation. This model was initially introduced in order to describe the bidimensional evolution of hydrodynamical wave packets [46]. It involves an auxiliary field $\psi$, which can be called a rectified field, because in optics it results from the optical rectification of the wave. This field describes a solitary wave, in the sense of an isolated oscillation, contrarily to an envelope soliton, i.e., there is no carrier.

The DS system (68) reduces obviously to the 2D NLS equation (67) if

$$
\left|\begin{array}{ll}
\alpha & \gamma \\
\beta & \delta
\end{array}\right|=\alpha \delta-\beta \gamma=0,
$$

but in this case only. It is thus more general than the $2 \mathrm{D}$ NLS equation. In fact, the DS system is the relevant model for most propagation problems for a single polarization in $(2+1)$ dimensions, as soon as a quadratic nonlinearity and an anisotropy are present.

The DS system is integrable by means of the IST method for some values of its coefficients. The multidimensional systems that possess this property are very rare [47]. Rigorously speaking, the system (68) is called DS when it is integrable only.

\subsection{The zero-order term}

The essential difference between a DS-type system (68) and the 2D NLS equation (67) above is the presence of the auxiliary field $\psi$ in DS. It usually represents the continuous component of the field, or its mean value, also called rectified field. In an expansion comparable to that of section 3.1, it often happens that the computation of the zero harmonic, and more 
precisely of the term $u_{2}^{0}$, presents some difficulties. These are the grounds for the appearance, in $(2+1)$ dimensions, of the second equation of the DS-type system. Let us see how it works on an example. I choose again as starting point an equation without physical significance, but allowing us to present the computations in the simplest way possible:

$$
u_{x x}+u_{y y}-u_{t t}=u_{x x x x}+\left(u^{2}\right)_{x x},
$$

where $u$ is expanded as in section 3.1, equation (31), with the slow variables defined by (65), without the third dimension $z$. At the beginning, the perturbation method applies in a way completely analogous to what has been seen in $(1+1)$ dimensions, starting from the system (28), (29). At order $\varepsilon$ we obtain the dispersion relation of the linearized equation, $\omega^{2}=k^{2}+k^{4}$. We set $u_{1}^{1}=f$. At order $\varepsilon^{2}$, the transport equation analogous to equation (41) fixes the value of the velocity $V$ as

$$
V=\frac{\mathrm{d} \omega}{\mathrm{d} k}=\frac{k}{\omega}\left(1+2 k^{2}\right) .
$$

The second harmonic at this order is computed without any difficulty: $u_{2}^{2}=f^{2} / 3 k^{2}$.

The changes appear in the computation of the zero harmonic $u_{2}^{0}$. The equations obtained at order $\varepsilon^{2}$ are analogous to equations (37) and (38) of section 3.1. Note that the lefthand-side member of the latter is zero when $p=0$, i.e. for the continuous component under consideration. In the present case, the right-hand-side member also contains derivatives only, and the corresponding equation is trivial. The equation that allows the computation of $u_{2}^{0}$ must then be sought at a higher order, here the order $\varepsilon^{4}$; it is the partial differential equation

$$
\left(1+V^{2}\right) \partial_{\xi}^{2} \psi+\partial_{\eta}^{2} \psi=\partial_{\xi}^{2}|f|^{2},
$$

where $\psi=u_{2}^{0}$. In $(1+1)$ dimensions, i.e. if the $\eta$ dependency is suppressed, it can be integrated to yield the explicit expression

$$
u_{2}^{0}=\psi=\frac{1}{1+V^{2}}|f|^{2} .
$$

In $(2+1)$ dimensions, this is no longer possible. At order $\varepsilon^{3}$ we obtain, in a way analogous to the $(1+1)$-dimensional case of section 3.1, the nonlinear evolution equation satisfied by the amplitude $f$ of the fundamental as

$$
2 \mathrm{i} \omega f_{\tau}+f_{\eta \eta}+P f_{\xi \xi}+\frac{2}{3} f|f|^{2}+2 k^{2} f \psi=0,
$$

with $P=1-V^{2}+6 k^{2}$. The set of equations (72), (74) is the asymptotic model which governs the evolution of the wave packet, it is of DS type. If the $\eta$ dependency is suppressed, it reduces to the NLS equation (66), with the coefficients $A=2 \mathrm{i} \omega, B=P$ and

$$
C=2 / 3+\frac{2 k^{2}}{1+V^{2}}
$$

This type of behaviour is very general, it can be generalized to $(3+1)$ dimensions and to models describing the non-resonant interaction of several polarizations. Applications to nonlinear optics, using nonlinear susceptibility tensors [48], and to electromagnetic wave propagation in a ferromagnetic medium [49] are presented in section 8.

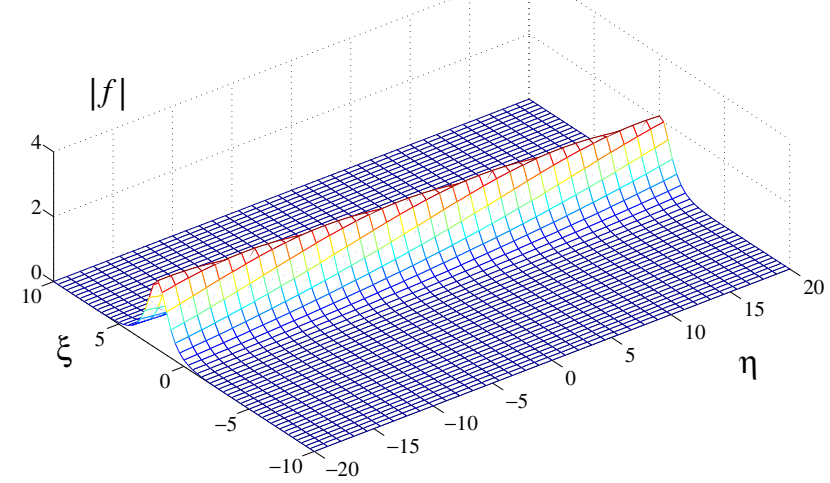

Figure 5. The line soliton of the DS system: it is the fundamental soliton given by the IST.

It should be noted that the DS system may arise more generally to account for the interaction between a solitary wave $\psi$ and a pulse envelope $f$. For example, it has been derived among other asymptotic models from the set of two coupled NLS equations which models a two-component Bose-Einstein condensate [50].

\subsection{Davey-Stewartson solitons}

Whatever the value of its coefficients is, the above DS-type system (68) can be written in the form

$\mathrm{i} f_{\zeta}+\varepsilon_{1} f_{\xi \xi}+f_{\eta \eta}+\varepsilon_{2} f|f|^{2}+v f \psi=0$,

$\psi_{\xi \xi}+\mu \psi_{\eta \eta}=|f|_{\xi \xi}^{2}$,

where $\mu$ and $\nu$ are real constants and $\varepsilon_{1}, \varepsilon_{2}= \pm 1$. The system (76) is integrable if and only if the two conditions

$$
\mu=-\varepsilon_{1}, \quad \nu=-2 \varepsilon_{2}
$$

are satisfied. There are thus four cases of integrability, depending on the signs of $\varepsilon_{1}$ and $\varepsilon_{2}$. Line solitons exist in all sign cases, cf figure 5. Solutions algebraically decreasing in all directions, called lumps, exist if $\varepsilon_{1} \varepsilon_{2}=-1$ [51]. The 1-lump solution is drawn in figure 6 . Solutions exponentially decreasing in all directions, called dromions, exist if $\varepsilon_{1}=+1$ [52-54], cf figure 7. In this case, the system DS is called DS I. The dromions involve non-trivial values of the auxiliary or rectified field $\psi$ at infinity, as drawn in figure 8 . These values can describe two incident solitary waves that interact with the main pulse. The physical interpretation of this interaction is detailed in section 8.4 .

\subsection{Transverse variations of the long waves}

It has been seen above that taking into account the transverse dimensions, when studying envelope solitons, describes 'spherical' wave packets, whose dimensions are of the same order in all directions. The situation radically differs if long waves are considered. Indeed the NLS-type descriptions involve three scales, the first of which is that of the wavelength $\lambda$. It is this scale, and more precisely the wave vector $\vec{k}$, which specifies the propagation direction. In the case of the 


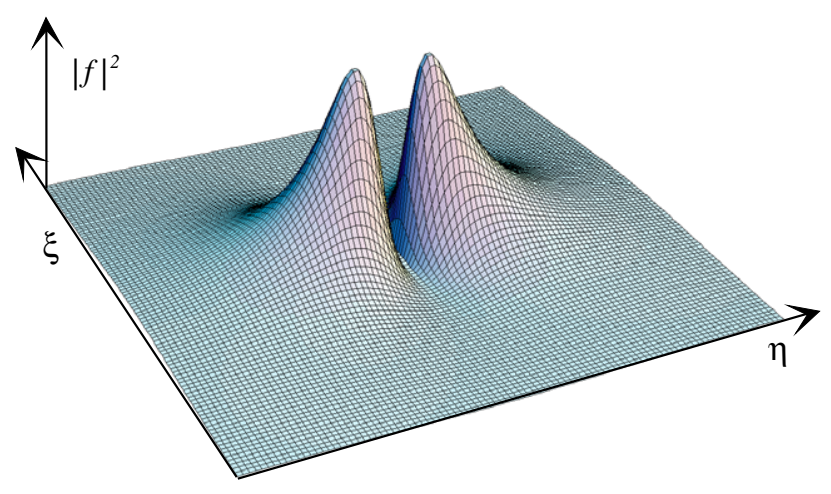

Figure 6. The lump solution of the DS system. It is an analytical solution algebraically decaying at infinity.

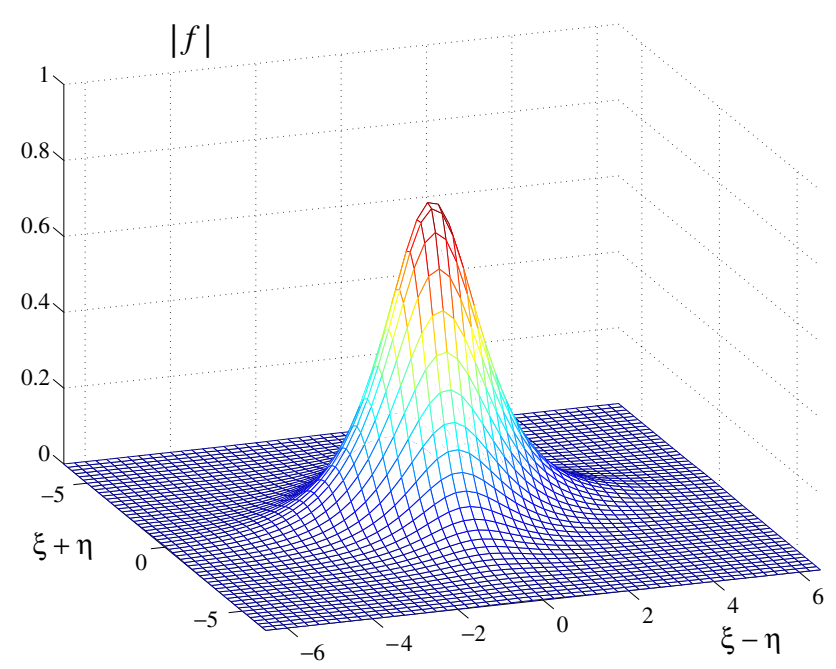

Figure 7. Dromion of the DS I system. It is a true tridimensional soliton, stable and exponentially decaying in all directions. It involves the particular shape of rectified field $\psi$ shown in figure 8 .

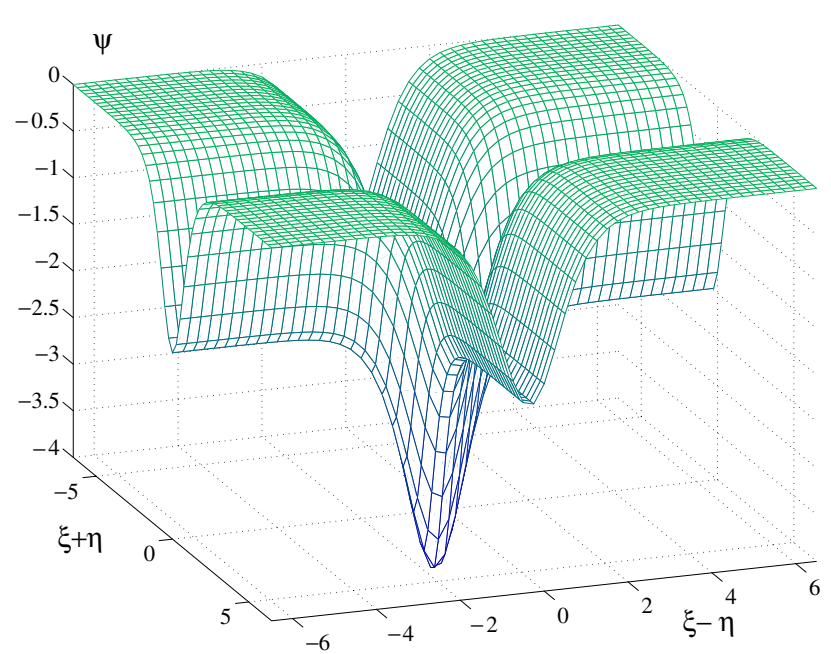

Figure 8. The rectified field $\psi$, corresponding to the dromion solution of the DS I system shown in figure 7.

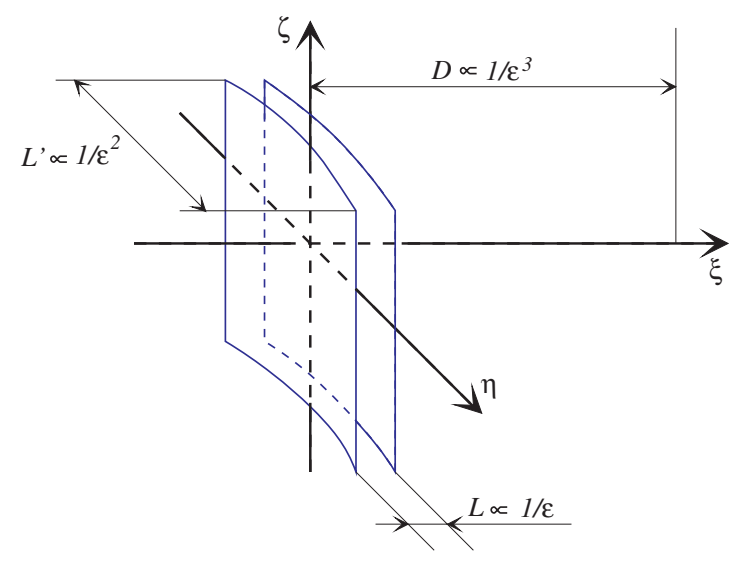

Figure 9. The various scales involved by the study of the transverse variations of the long waves. $L$ : length of the solitary wave, $L^{\prime}$ : its transverse extension, $D$ : propagation distance, with $1 \ll L \ll L^{\prime} \ll D$.

long waves, there are only two scales left. The long-wave approximation can be defined as a limit of oscillating waves, whose wavelength $\lambda$ tends to infinity. The oscillation of the carrier then becomes very slow and merges with the variable $\xi=\varepsilon(x-V t)$ which describes the shape of the wave packet. This gives an interpretation of the reduction of the number of scales. There is no preferential direction defined by $\vec{k}$ any more; this direction is now fixed by the variable $\xi$ of order $\varepsilon$.

It is clear that if we introduce the transverse variables $\varepsilon y, \varepsilon z$ of the same order as $\xi$, we will not give rise to the transverse evolution in the $y$ and $z$ directions of a wave propagating along the $x$-axis, but merely allow this wave to propagate in any direction. In order to study the evolution of the wave with respect to transverse perturbations, one must first define its propagation direction, that is the direction of the faster variation. Hence the transverse variables must be 'slower' than $\xi$, and we will have an ansatz of the type

$$
\xi=\varepsilon(x-V t), \quad \eta=\varepsilon^{2} y, \quad \zeta=\varepsilon^{2} z, \quad \tau=\varepsilon^{3} t .
$$

This means that the longitudinal extension of the wave will be $L \propto 1 / \varepsilon$, its transverse extension $L^{\prime} \propto 1 / \varepsilon^{2}$, very large with regard to $L$, as illustrated in figure 9 . The asymptotic model which can be obtained with this type of scaling is typically the Kadomtsev-Petviashvili (KP) equation:

$$
\partial_{\xi}\left(\partial_{\tau} f+\mu f \partial_{\xi} f+v \partial_{\xi}^{3} f\right)+\rho \partial_{\eta}^{2} f=0
$$

Equation (79) is integrable by means of the IST method. The scaling (78) has been introduced first for magnetosonic waves [55]. However, the KP equation (79) is essentially known for describing the evolution of hydrodynamical surface waves $[7,56]$. In this situation, the real coefficients $\mu, v$ and $\rho$ are

$\mu=\frac{3 V}{2 h}, \quad v=V\left(\frac{h^{2}}{6}-\frac{T}{2 \rho_{0} g}\right), \quad \rho=\frac{V}{2}$,

where $g$ is the gravity acceleration, $h$ is the depth of the pond, $T$ is the surface tension of the fluid, $\rho_{0}$ its specific mass, $f$ is the height of its free surface and $V=\sqrt{g h}$ is the wave velocity. When the effect of gravity dominates, the coefficient $v$ is positive, and equation (79) is called KP II. It admits 


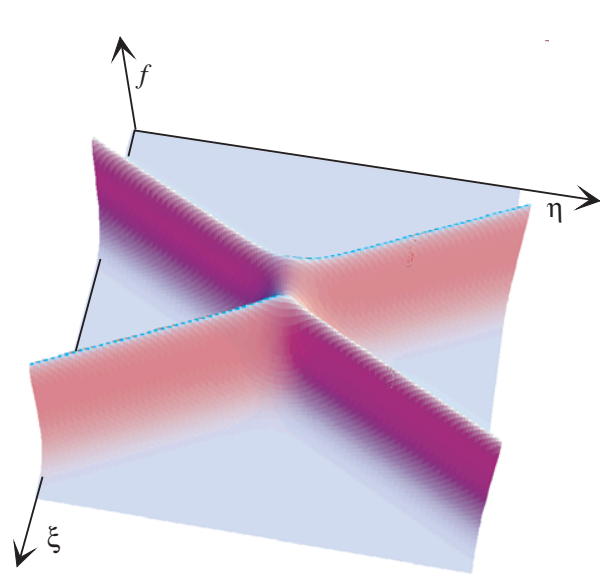

Figure 10. Interaction of two line soliton solutions of the Kadomtsev-Petviashvili equation. They undergo a shift in position at the interaction point.

line soliton-type solutions, which are stable, but no localized solution. The interaction between two line solitons is drawn in figure 10. It has been observed at sea. When the surface tension dominates, what happens is that, in water at ordinary temperatures, for very small values of the depth $h$, less than about $5 \mathrm{~mm}, v$ is negative, and equation (79) is called KP I. In this case the line solitons are unstable; on the other hand the equation admits localized algebraically decreasing solutions, called lumps [51, 57].

\section{Higher orders and KdV hierarchy}

\subsection{Corrections to the long-wave approximation}

A large number of nonlinear partial differential equations can be reduced, by means of the multiscale expansion method, to the $\mathrm{KdV}$ equation. The conditions have been clarified, $\mathrm{cf}$ section 2.1 and [32]. In other words, KdV solitons can exist in a large number of physical situations. From another point of view, the IST method allows us to solve the asymptotic model $\mathrm{KdV}$ and gives thus an approximate solution of the starting problem, in a very general way.

This first approximation of the solution is acceptable in special situations only, in which the orders of magnitude of the amplitude, of the solitary wave dimensions, and of the propagation distance or time are specified. As soon as we depart from these values, corrections become necessary. As long as the order of magnitude of the propagation time is not modified, these corrections are taken into account in a way completely analogous to a classical perturbation expansion, introducing terms of higher order with respect to the perturbative parameter $\varepsilon$. These corrections are solutions of a $\mathrm{KdV}$ equation linearized about the leading term, with a right-hand-side member depending on the previous orders, such as e.g. equation (85) below. The KdV asymptotic involves a slow time variable $\tau=\varepsilon^{3} t$. It is hence expected to be valid for a finite value $\tau_{0}$ of $\tau$ : the corresponding physical time is $t_{0}=\tau_{0} / \varepsilon^{3}$, and hence $\mathrm{KdV}$ is valid on propagation distances or times with the same order of magnitude as $1 / \varepsilon^{3}$. When one wants to describe the propagation on longer distances or times, further difficulties arise. Indeed, as has been noticed above, a small correction, cumulated over a long distance, can produce a large effect. The solutions of the linearized KdV equation present terms called secular, which increase linearly with time. Let us consider a small correction, say of order $\varepsilon^{p}$. If it increases linearly during a very long time, of order $1 / \varepsilon^{q}$, it becomes obviously a quantity of order $\varepsilon^{p-q}$. It is thus very large if $q$ is greater than $p$. To avoid this, we impose that the correction solutions of the linearized $\mathrm{KdV}$ equation are bounded or at least grow slower than linearly with time (such a behaviour is called 'sublinear'). In other words, we arrange that these secular terms vanish. The latter are characterized by particular source terms in the right-hand-side member. Hence we have to set conditions to be satisfied by the previous orders, in such a way that these secular-producing terms vanish. Note that this reasoning is exactly the same as we used in section 3.1 in order to derive the NLS equation.

\subsection{The secular solutions of the linearized $K d V$ equation}

The inhomogeneous linearized $\mathrm{KdV}$ equation can be solved by means of the inverse scattering transform method [58, 59]. The secular-producing terms in the right-hand-side member of the equation have been characterized in several ways, and first, as resonant terms. The $\mathrm{KdV}$ equation is written in the form

$$
\partial_{t} f-6 f \partial_{x} f+\partial_{x}^{3} f=0 .
$$

Denote by $\phi_{k}$ and $\psi_{k}$ the Jost functions [6], solutions of the scattering equation associated with $\mathrm{KdV}$, with

$$
\phi_{k x} \stackrel{\sim}{\rightarrow}+\infty \mathrm{e}^{+\mathrm{i} k x}, \quad \psi_{k x} \stackrel{\sim}{\rightarrow}-\infty \mathrm{e}^{+\mathrm{i} k x},
$$

where $k$ is a real or a purely imaginary root of the scattering data $a(k)$, denoted by $k=\mathrm{i} \kappa_{l}(l=1,2, \ldots, n)$. Further, we define $\Phi_{k}=\phi_{k}^{2}$ and $\Psi_{k}=\partial_{x}\left(\psi_{k}^{2}\right)$. The functions

$$
\Phi_{k} \mathrm{e}^{-8 \mathrm{i} k^{3} t} \quad \text { and } \quad \Psi_{k} \mathrm{e}^{-8 \mathrm{i} k^{3} t}
$$

satisfy the homogeneous linearized $\mathrm{KdV}$ equation. The solution of the Cauchy problem for the inhomogeneous equation is obtained by an explicit expansion on the basis of the functions $\Phi_{k}$ or on that of the functions $\Psi_{k}$.

In order to characterize the secular-producing terms, we expand the right-hand-side member onto the functions $\Phi_{k}$. The coefficients are functions of time, which can be expanded in a way that generalizes in some sense a Fourier transform. One of the components oscillates with the pulsation $8 \mathrm{i} k^{3}$ as the solution (83) of the homogeneous linearized $\mathrm{KdV}$ equation does, or decreases as $\mathrm{e}^{-8 \kappa_{j}^{3} t}$, if $k=\mathrm{i} \kappa_{j}$ belongs to the discrete spectrum. This term is resonant and is the source of a secular term. The right-hand-side members that are not secularproducing are thus characterized in the following way: for each component on $\Phi_{k}$, the sub-component of it that evolves in the same way as the solution of the homogeneous equation must be zero.

Second, the secular-producing terms are interpreted as conserved densities of the $\mathrm{KdV}$ equation. The latter can be written with the help of the Jost functions $\psi_{k}$ as [58]

$$
\begin{gathered}
\mathcal{A}_{j}(x, t)=2(-1)^{j} \sum_{l=1}^{n} \kappa_{l}^{2 j-1} C_{l}(t) \psi_{l}^{2}(x, t) \\
+\frac{\mathrm{i}}{\pi} \int_{-\infty}^{+\infty} k^{2 j-1} r(k, t) \psi_{k}^{2}(x, t) \mathrm{d} k,
\end{gathered}
$$


where $C_{l}(t)$ and $r(k, t)$ are, respectively, the normalization coefficient of the bound states and the reflection coefficient. They characterize the scattering data. We deduce from (84) that the derivative $\partial_{x} \mathcal{A}_{j}(x, t)$ of a conserved density is a linear combination and integral of $\Psi_{k}$, and the temporal evolution of the scattering data is exactly the one which ensures that this combination represents a solution of the homogeneous linearized $\mathrm{KdV}$ equation. After a study of the reciprocal property, the secular-producing terms can be identified with the spatial derivatives of the conserved densities [59].

\subsection{Higher-order times and the KdV hierarchy}

Let $T_{0}, L_{0}, A_{0}$ be a time, a length, and a value of the wave amplitude, characteristic for the system. According to figure 1, the perturbative parameter $\varepsilon$ is defined in such a way that the length of the solitary wave is $L_{0} / \varepsilon$ and its amplitude $\varepsilon^{2} A_{0}$. As written above, the $\mathrm{KdV}$-type asymptotic is valid for times up to $T_{0} / \varepsilon^{3}$. Small corrections to this asymptotic, cumulated over very long times, can indeed modify greatly the behaviour of the wave. In order to reach larger values of the time variable, we pursue the perturbative approach, following the idea of Kraenkel, Pereira and Manna [60-62], introducing higherorder time variables $\tau_{j}=\varepsilon^{2 j+1} t, j=1,2, \ldots$ In other words, we still have some starting model, e.g. equation (2), and we are looking for an approximate model with the help of expansion (4), in which we assume that the profiles depend not only on the slow variables defined by (5) but also on higher-order time variables $\tau_{1}=\tau, \tau_{2}, \tau_{3}$, etc. The leading term is characterized by an amplitude, denoted by $g$ in section 2.1, and which will be denoted here by $\varphi_{2}$ in order to recall that it is a quantity of order $\varepsilon^{2}$. The expansion will also involve higher-order terms, described by the amplitudes $\varphi_{4}, \varphi_{6}$, etc, corresponding to corrections of order $\varepsilon^{4}, \varepsilon^{6}$, etc (the expansion must have a certain parity). The equation giving the evolution of the leading term $\varphi_{2}$ with regard to $\tau_{3}$ is obtained by imposing that the following term $\varphi_{4}$ is bounded or more exactly sublinear. The equation which determines the evolution of this term is

$$
\partial_{\tau_{1}} \varphi_{4}+P \partial_{\xi}\left(\varphi_{2} \varphi_{4}\right)+Q \partial_{\xi}^{3} \varphi_{4}=\partial_{\tau_{2}} \varphi_{2}-\gamma_{2} \partial_{\xi}^{5} \varphi_{2}+\mathcal{O}_{2}
$$

Here $\mathcal{O}_{2}$ refers to an expression depending on $\varphi_{2}$, without a linear term, and $\gamma_{2}$ is a real coefficient, which can be computed $[63,64]$. The condition to be satisfied is thus that equation (85) does not admit any secular solution. Through an explicit computation in the case where $\varphi_{2}$ is the one-soliton solution of the KdV equation, Kodama and Taniuti [58] have noticed that the secular-producing terms are those linear with regard to the solution of lowest order $\varphi_{2}$. The secular solutions $\varphi_{4}$ will thus vanish if the linear terms vanish from the right-hand-side member of equation (85). To achieve this, we impose that $\varphi_{2}$ satisfies some partial differential equation such that

$$
\partial_{\tau_{2}} \varphi_{2}=-\gamma_{2} \partial_{\xi}^{5} \varphi_{2}+\mathcal{O}_{2}
$$

We still need to determine the nonlinear terms of equation (86), represented by $\mathcal{O}_{2}$. They are not free but imposed by the compatibility condition between the KdV equation (19) and equation (86), which is the Schwartz condition $\partial \tau_{1} \partial \tau_{2} \varphi_{2}=$ $\partial \tau_{2} \partial \tau_{1} \varphi_{2}$. The only equation which possesses the same homogeneity properties as the right-hand-side member of (85), and which satisfies this condition, is the second equation of what is called the KdV hierarchy [62, 32].

The KdV hierarchy is the family of equations [65]

$$
\partial_{T_{n}} g=\partial_{X} \mathcal{L}^{n} g \quad(n \text { integer })
$$

where $\mathcal{L}$ is a recurrence operator defined by

$$
\mathcal{L}=-\frac{1}{4} \partial_{X}^{2}-g+\frac{1}{2} \int^{X} \mathrm{~d} X\left(\partial_{X} g\right) .
$$

For $n=1$, it is the $\mathrm{KdV}$ equation, with a normalization different from that of equation (19) ( $P$ is replaced with $\frac{3}{2}$, $Q$ with $\frac{1}{4}$ ). We identify it with equation (19) using the relations

$$
g=\frac{P}{6 Q} \varphi_{2}, \quad X=\xi \quad \text { and } \quad T_{1}=4 Q \tau_{1} .
$$

For $n=2$, the equation of the hierarchy (87) is

$$
\partial_{T_{2}} g=\frac{1}{16} \partial_{X}^{5} g+\frac{5}{4}\left(\partial_{X} g\right) \partial_{X}^{2} g+\frac{5}{8} g \partial_{X}^{3} g+\frac{15}{8} g^{2} \partial_{X} g .
$$

An important property is the existence of the $\tau$ Hirota function [66], which is a function of all variables $\left(X, T_{1}, T_{2}, \ldots\right)$, related to $g$ through

$$
g\left(X, T_{1}, T_{2}, \ldots\right)=2 \partial_{X}^{2} \ln \tau\left(X, T_{1}, T_{2}, \ldots\right)
$$

(to avoid any confusion between the $\tau$ Hirota function and the time variables $\tau_{j}$ ). The existence of $\tau$ ensures that a solution $g$ of the system yielded by all equations of the hierarchy exists and hence that the Schwartz condition is satisfied at any order. After an adequate choice of the proportionality constant $\gamma_{2}$, the time variables of order 2 (the variable $\tau_{2}$ of the expansion and the variable $T_{2}$ of the hierarchy) are connected by

$$
T_{2}=-16 \gamma_{2} \tau_{2}
$$

The evolution equation to be satisfied by $\varphi_{2}$ is thus

$$
\frac{-1}{16 \gamma_{2}} \partial_{\tau_{2}} \varphi_{2}=\partial_{\xi} \mathcal{L}^{2} \varphi_{2}
$$

This way, the linear terms have been removed from equation (86). It remains to justify that this procedure, that removes all linear terms from the right-hand-side member of the linearized $\mathrm{KdV}$ equation, assuming it to be polynomial with regard to the solution of $\mathrm{KdV}$, ensures that the solution of the linearized equation is bounded [59]. Considering the relations existing between the conserved densities $\mathcal{A}_{j}$ and the recurrence operator $\mathcal{L}$, defined by (88), which allows us to write the hierarchy, it is possible. Indeed, it is easily seen that when the linear terms are removed using the above procedure, the terms proportional to $\partial_{x} \mathcal{A}_{j}$ exactly are removed. And these terms are the secular-producing ones, as seen above.

On the other hand, the right-hand-side member of the linearized $\mathrm{KdV}$ equation which governs the evolution of $\varphi_{6}$ involves $\varphi_{4}$, a solution of (85). It is thus necessary to see, when a solution of the linearized $\mathrm{KdV}$ equation itself is used in the right-hand-side member, which part of it is secularproducing and which part is not. This is not too difficult. Indeed, this solution is given by its expansion on the basis of $\Phi_{k}$, and we have characterized the fact that a source term is secular-producing or not by some criterion, which involves the coefficients of this expansion and their $t$-dependency. A last point remains to be considered: the dependency of the 
higher-order terms with regard to the higher order times, it is governed by a linearized KdV hierarchy [32]. Finally, we can justify that the higher-order terms are not secular-producing and prove that the formal expansion contains bounded terms only.

\subsection{The soliton of the hierarchy}

Repeating the same procedure at any order, the equation governing the evolution of $v$ with respect to any time variable $T_{n}$ is equation (87) of the hierarchy, with

$$
T_{n}=-(-4)^{n} \gamma_{n} \tau_{n},
$$

where $\gamma_{n}, n=3,4, \ldots$, are time scaling coefficients. As mentioned above, all equations of the KdV hierarchy are compatible together, in the sense that for any given initial data a function $g\left(X, T_{1}, T_{2}, T_{3}, \ldots\right)$ satisfying equation (87) for any value of $n$ can be found. This solution can be found using the inverse scattering transform (IST) method, at least in principle [63]. Indeed, all equations of the hierarchy are completely integrable by means of the IST method. Furthermore, they can all be described in the IST formalism using the same spectral problem [6], which ensures their compatibility. The scattering data $\left(R_{+}(k), D_{+, j}, k_{j}\right)$ are defined in the same way for all equations, only their time evolution differs for each time variable $T_{n}$. The time evolution is given by

$$
\begin{gathered}
R_{+}\left(T_{n}\right)=R_{+}(0) \mathrm{e}^{\Omega_{n}(k) T_{n}}, \\
D_{+, j}\left(T_{n}\right)=D_{+, j}(0) \mathrm{e}^{\Omega_{n, j} T_{n}}, \\
k_{j}\left(T_{n}\right)=k_{j}(0) .
\end{gathered}
$$

The index $n$ refers to the $n$th equation of the hierarchy. The evolution factors are $\Omega_{n, j}=\Omega_{n}\left(k_{j}\right)$ and $\Omega_{n}(k)=-\mathrm{i} \omega_{n}(2 k)$, where $\omega_{n}(k)$ is the dispersion relation of the $n$th equation of the hierarchy linearized. It is seen from relation (96) that the discrete spectrum $\left(k_{j}\right)$ is constant with regard to any of the time variables $T_{n}$. Therefore, the number of solitons and their characteristics are not modified by the higher-order time evolution. The evolution of the spectral data with regard to all the higher-order time variables can then be written as a single exponential factor for each spectral component,

$$
R_{+}\left(T_{1}, T_{2}, \ldots\right)=R_{+}(0,0, \ldots) \exp \left(\sum_{n=1}^{\infty} \Omega_{n}(k) T_{n}\right) .
$$

Using expressions (87), (88) of the equations of the hierarchy, the expression of the complete time evolution factor is reduced to

$$
\sum_{n=1}^{\infty} \Omega_{n, j}(k) T_{n}=\Omega_{j} t, \quad \text { with } \quad \Omega_{j}=\sum_{n=1}^{\infty}\left(2 \varepsilon \kappa_{j}\right)^{2 n+1} \gamma_{n} .
$$

Obviously formula (98) is valid only if the power series converges. Note that the coefficients of the latter are the time scaling coefficients $\gamma_{n}$. For a one-soliton solution, the above formulae show that the introduction of a sequence of higherorder time variables and of all equations of the $\mathrm{KdV}$ hierarchy
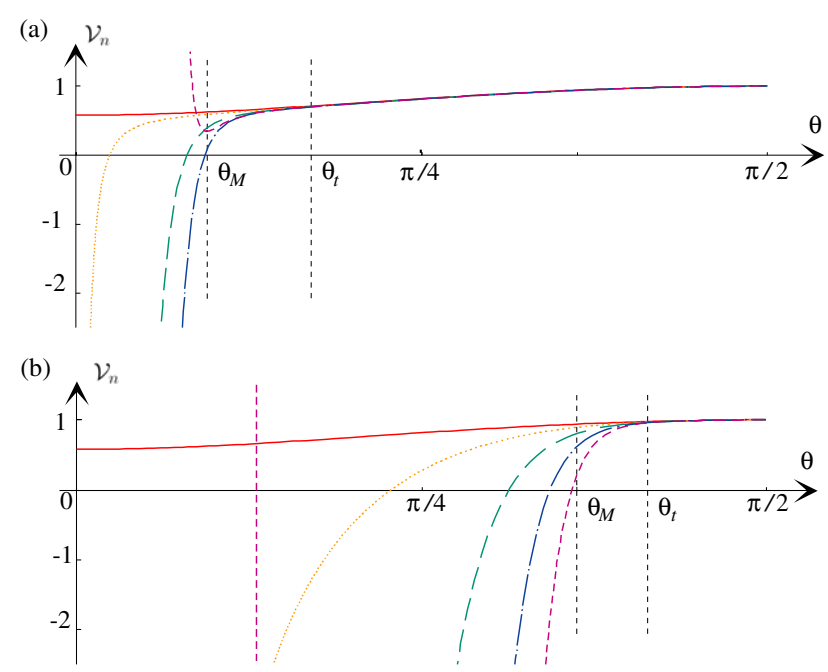

Figure 11. Plot of the five first approximate values $\mathcal{V}_{n}=V+\sum_{p=1}^{n}\left(\beta / \varepsilon_{n}\right)^{2 n}$ of the velocity $\mathcal{V}$ of the soliton of the full $\mathrm{KdV}$ hierarchy, against the angle $\theta$ between the propagation direction and the exterior field. Solid line: $n=0$, dotted line: $n=1$, large dashed: $n=2$, dashed-dotted line: $n=3$, short dashed: $n=4$. For a soliton parameter, $\beta=0.1$ (a), $\beta=1.5$ (b). For $\theta>\theta_{t}$, all curves are superposed: the first approximation (KdV) is valid. For $\theta_{M}<\theta<\theta_{t}$, the series converges: a $\mathrm{KdV}$ approximation with higher-order terms is valid. For $\theta<\theta_{M}$, the series does not converge: the KdV-type soliton does not exist.

yield nothing but a renormalization of the soliton speed $[63,33]$,

$$
g=2 b^{2} \operatorname{sech}^{2} b\left(X+\sum_{n=1}^{\infty}\left(-b^{2}\right)^{n} T_{n}\right),
$$

with $b$ being the soliton parameter. In the case of magnetic solitons [64], it can be written using the physical variables as

$$
\vec{H}_{\mathrm{w}}=\frac{12 Q}{P} \vec{h}_{1} \beta^{2} \operatorname{sech}^{2} \beta(x-\mathcal{V} t)
$$

where

$$
\mathcal{V}=V+\sum_{n=1}^{\infty} 4^{n} \beta^{2 n} \gamma_{n}
$$

where $V$ is the group velocity, $\vec{h}_{1}$ is the polarization vector, $\vec{H}_{\mathrm{w}}$ is the wave magnetic field and $\beta$ is the dimensional soliton parameter, $\beta=\varepsilon b$.

The convergence of the perturbative scheme is closely related to the convergence of the power series (101) defining the velocity $\mathcal{V}$. An example of computation showing the first partial sums of the latter, against the angle $\theta$ between the propagation direction and the applied field, is drawn in figure 11. It is seen that, for $\theta$ close to $\pi / 2$, the first approximation $(\mathrm{KdV})$ gives almost the exact speed, while for small angles the series diverges. When $\theta$ is less than some threshold value $\theta_{M}$, the series does not converge and the whole perturbative approach is not valid. When $\theta>\theta_{M}$ (insofar that we can deduce it from the soliton speed only), the $\mathrm{KdV}$ approximation will correctly describe the wave evolution. Moreover, the $\mathrm{KdV}$ equation itself will give an acceptable description above some value $\theta_{t}$ of the angle $\theta$, while this 
first-order approximation needs to be corrected by higher-order terms below $\theta_{t}$ (note that the threshold value $\theta_{M}$ is precisely defined, while $\theta_{t}$ is only an order of magnitude depending on the accuracy required). It is reasonable to think that the same kind of conclusion holds in a more general situation, involving several solitons and radiation.

\section{Long waves in ferromagnetic media}

\subsection{Introduction: linear theory and envelope solitons}

The evolution of a magnetic momentum $\vec{M}$ in a magnetic field $\vec{H}$ is described by the equation

$$
\partial_{t} \vec{M}=-\delta \mu_{0} \vec{M} \wedge \vec{H},
$$

where $\delta$ is the gyromagnetic ratio $(\delta>0)$ and $\mu_{0}$ is the magnetic permeability in vacuum. In a ferromagnetic medium, this relation remains valid in first approximation for the magnetization density $\vec{M}$ of the medium. A more accurate model is obtained by replacing the field $\vec{H}$ by an effective field, which contains several additional terms accounting for the inhomogeneous exchange interaction, the anisotropy, and by adding to the equation a phenomenological term which accounts for the damping. This model is due to Landau and Lifschitz, and takes their name. If the field $\vec{H}$ is constant, equation (102) is easily solved: $\vec{M}$ rotates around $\vec{H}$, with a pulsation $\omega_{\mathrm{L}}=\delta \mu_{0} H$, corresponding to the Larmor precession $(H$ is the norm of $\vec{H})$. An incident wave with the same frequency will be able to resonate with this eigenmode: it is the ferromagnetic resonance. However, the resonance pulsation $\omega_{\mathrm{R}}$ is equal to $\omega_{\mathrm{L}}$ only if the wave propagates parallel to the magnetic field. In the other cases, it is higher and takes at most the value $\delta \mu_{0} \sqrt{H(H+M)}$, when the propagation is perpendicular to the field. We commonly use the following parameters: the saturation magnetization $m$ and the ratio $\alpha=H / m$ of the applied magnetic field to it. Indeed, a uniform magnetic field is usually applied to the sample, in order to saturate the magnetization. From the experimental point of view, this allows us to avoid that the domain walls perturb the wave propagation. From the theoretical point of view, the presence of a static term is a necessary condition to make use of a perturbative approach. Strictly speaking, the field $\vec{H}$ which we call 'static' or 'external' is not the applied field, but the magnetic field effectively created by it inside the material. Otherwise, demagnetizing factors should be taken into account. With these notations, the ferromagnetic resonance frequency $\omega_{\mathrm{R}}$ ranges from $\delta \mu_{0} m \alpha$ to $\delta \mu_{0} m \sqrt{\alpha(1+\alpha)}$.

The evolution of the magnetic field $\vec{H}$ is described by the Maxwell equations. We assume that, regarding its dielectric properties, the material is perfectly linear and isotropic, and we denote by $c=1 / \sqrt{\hat{\varepsilon} \mu_{0}}$ the light velocity based on its dielectric constant $\hat{\varepsilon}$. The Maxwell equations then reduce to

$$
-\vec{\nabla}(\vec{\nabla} \cdot \vec{H})+\Delta \vec{H}=\frac{1}{c^{2}} \partial_{t}^{2}(\vec{H}+\vec{M}) .
$$

The system yielded by the Landau-Lifschitz equation (102) and the wave equation (103) describes electromagnetic wave propagation in a ferromagnetic medium $[67,68] . \vec{H}, \vec{M}$

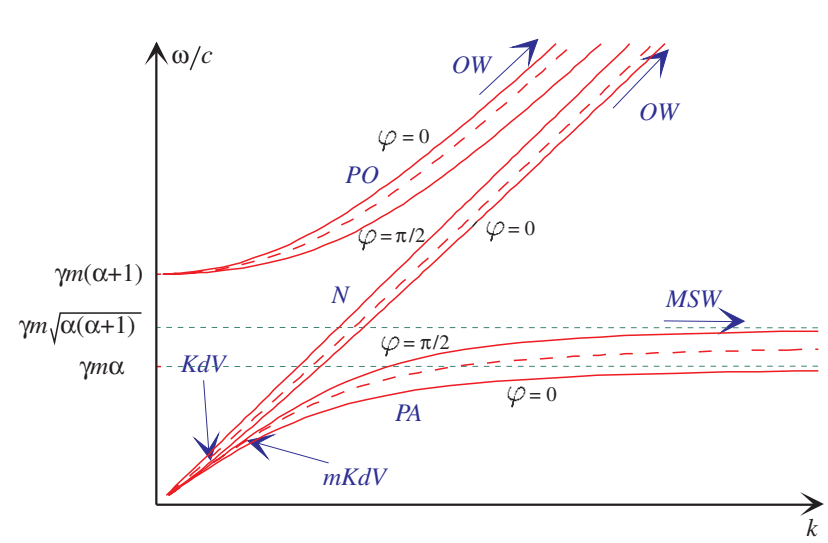

Figure 12. Dispersion relation of the electromagnetic waves in ferromagnetic media, with the indication of the various modes, and for several values of the angle $\varphi$ between the propagation direction and the applied magnetic field. $O W$ : optical waves, $M S W$ : magnetostatic waves. The proper electromagnetic waves are $P O$ : optical with positive helicity, $P A$ : acoustic with positive helicity, $N$ : with negative helicity. KdV, mKdV: long-wave modes governed by the $\mathrm{KdV}$ and by the modified $\mathrm{KdV}$ equations, respectively. $m=\left(\delta \mu_{0} / c\right) M_{s}(\mathrm{USI})$.

and $t$ are replaced below by the normalized quantities $\delta \mu_{0} \vec{H} / c, \delta \mu_{0} \vec{M} / c$ and $c t$, then the constants $\delta \mu_{0}$ and $c$ take the value 1 . Among the waves of electromagnetic nature that can propagate in a ferromagnetic medium, there are so-called optical waves, denoted by $O W$ in figure 12 , whose frequency is large enough with respect to the Larmor frequency $\omega_{\mathrm{L}} / 2 \pi=\delta \mu_{0} H / 2 \pi$ so that the magnetic effects can be neglected. Magnetostatic waves, $M S W$ in figure 12, are intensively studied [69]. Very close to the resonance frequency, the phase velocity $\omega / k$ and the group velocity $\mathrm{d} \omega / \mathrm{d} k$ of the wave become very small with regard to the light velocity. The equations describing the propagation of the wave become that of the magnetostatic, the propagation at the light velocity can indeed be considered as instantaneous and the retardation terms in the Maxwell equations can be neglected. Most of the experimental studies about solitons in ferromagnetic media concern magnetostatic waves and moreover guided modes of them in thin films [70-74].

We here consider electromagnetic waves, or polaritons, for which neither the retardation nor the magnetic effects are negligible [75]. Regarding the temporal solitons in (1+1) dimensions, focusing and defocusing regimes are distinguished [76-78]. Following the standard procedure presented in section 3.1, a multiscale expansion leads to a NLS-type asymptotic model,

$$
\mathrm{i} A f_{\tau}+B f_{\xi \xi}+C f|f|^{2}=0 .
$$

The amplitude $f$ is a function of the slow variables $\xi=$ $\varepsilon(x-V t)$ and $\tau=\varepsilon^{2} t$, and describes the wave envelope. Precisely, the magnetization is written as

$$
\vec{M}=\vec{M}_{0}+\varepsilon f \vec{m}_{1}^{1} \mathrm{e}^{\mathrm{i}(k x-\omega t)}+\text { c.c. }+O\left(\varepsilon^{2}\right),
$$

where $\vec{m}_{1}^{1}$ is a polarization vector defined by

$$
\vec{m}_{1}^{1}=\left(\begin{array}{l}
-\mathrm{i} \gamma \mu m \sin \varphi \\
\mathrm{i} \gamma \mu m \cos \varphi \\
-\gamma^{2} \omega
\end{array}\right),
$$




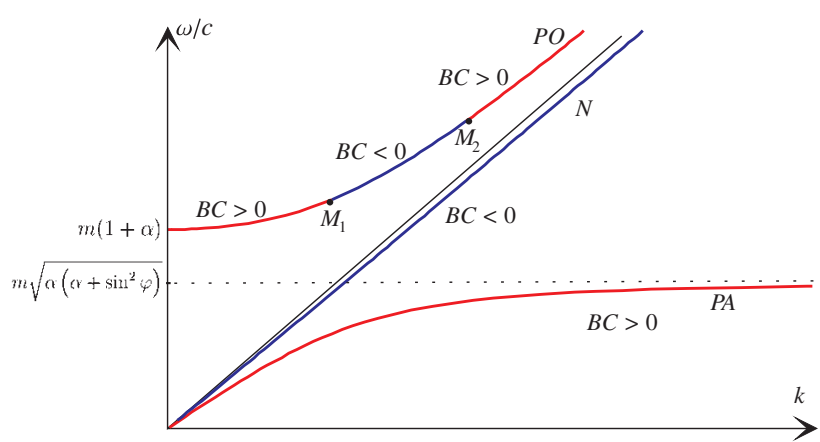

Figure 13. Schematic representation of the domains where the modulation of an electromagnetic wave is either stable $(B C<0)$, or unstable $(B C>0)$, on a plot of the dispersion relation.

where we have set, in normalized units, $\gamma=1-k^{2} / \omega^{2}$ and $\mu=1+\alpha \gamma$. As mentioned above, $\alpha$ is the ratio of the applied magnetic field to the saturation magnetization $m$, and $\varphi$ is the angle between the static uniform magnetization $\vec{M}_{0}=(m \cos \varphi, m \sin \varphi, 0)$ and the propagation direction $x$. The pulsation $\omega$ and the wave vector $k$ satisfy the dispersion relation

$$
\mu^{2} m^{2} \cos ^{2} \varphi+\gamma \mu(1+\alpha) m^{2} \sin ^{2} \varphi=\gamma^{2} \omega^{2},
$$

drawn in figure $12 . V=\mathrm{d} \omega / \mathrm{d} k$ is the group velocity.

Let us recall the well-known Lighthill criterion: if the product $B C$ of the coefficients of the nonlinear term and of the dispersive term is positive, a periodic modulation of the envelope $f$ is unstable [79], and soliton formation can occur [9], while if $B C$ is negative, the modulation is stable, but the solitons do not exist. It is known that in contrast dark soliton formation can occur in this case [80]. The question that arises is to determine the sign of the product $B C$ as a function of the physical parameters. In the particular case where the propagation direction is parallel to the uniform field, it is seen that the waves with positive helicity, corresponding to the branches $P O$ and $P A$ on the representation of the dispersion relation (figure 12), undergo a modulational instability and can yield solitons $(B C>0)$, while the waves with negative helicity, i.e. the branch $N$, are modulationally stable $(B C<0)$. When the propagation direction is not parallel to $\vec{M}_{0}$ any more, modulationally stable regions appear for the waves with positive helicity, as drawn in figure 13 [77]. The effect of the damping on the wave packets [81] and the interaction of the two modes (nonlinear Faraday effect) [40] have been considered.

\subsection{KdV-type solitons}

Consider the 'long-wave' limit of a wave with negative helicity $N$. As illustrated by the dispersion relation (figure 12), this wave propagates at the velocity $\sqrt{\left(\alpha+\sin ^{2} \varphi\right) /(\alpha+1)}$, where $\varphi$ is the angle between the propagation direction and the applied field and $\alpha$ is the ratio of the latter to the saturation magnetization. It is shown, using the slow variables

$$
\xi=\varepsilon(x-V t), \quad \tau=\varepsilon^{3} t,
$$

that the propagation of this type of 'long waves' is governed by the KdV equation [31]:

$$
g_{\tau}+P g g_{\xi}+Q g_{\xi \xi \xi}=0,
$$

where $P$ and $Q$ are real known constants and $g$ is the wave amplitude: the wave magnetic field and the magnetization are both proportional to $\varepsilon^{2} g$.

This assumes a negligible damping. The latter can be taken into account by modifying the Landau equation (102) as

$$
\partial_{t} \vec{M}=-\delta \mu_{0} \vec{M} \wedge \vec{H}+\frac{\sigma}{\|\vec{M}\|} \vec{M} \wedge(\vec{M} \wedge \vec{H})
$$

where $\sigma$ is a negative damping constant, $\delta$ is the gyromagnetic ratio and $\mu_{0}$ is the magnetic permeability in vacuum. We use below normalized quantities, so that the constants are replaced with one. A multiscale expansion involving the slow variables

$$
\xi=\varepsilon(x-V t), \quad \tau=\varepsilon^{2} t,
$$

leads to the Burgers' equation [27]

$$
g_{\tau}+P g g_{\xi}+R g_{\xi \xi}=0
$$

as an asymptotic model. Here $P$ has the same value as in equation (109) and $R$ is a real constant. Explicit computation shows that $P$ is positive and $R$ negative. Here, the magnetic field and the magnetization are proportional to $\varepsilon g$. Recall that the Burgers' equation (112) can be linearized by means of the so-called Hopf-Cole transformation $[6,2]$. A linear scaling for the time $\tau$ and the amplitude $g$ reduces the problem to $P=1$ and $R=-1$. The Hopf-Cole transformation is

$$
g=-2 \partial_{\xi} \ln F,
$$

then $F$ is a solution of the heat equation

$$
F_{\tau}=F_{\xi \xi} .
$$

If we choose for $F$ a linear combination of exponentials, we obtain the particular solution

$$
g=\frac{2 \sum_{i=1}^{n} f_{i} v_{i} \mathrm{e}^{-v_{i}\left(x-v_{i} t\right)}}{1+\sum_{i=1}^{n} f_{i} \mathrm{e}^{-v_{i}\left(x-v_{i} t\right)}},
$$

which describes the propagation of $n$ shock profiles and their coalescence, cf figure 14. A generalization to $(2+1)$ dimensions [27] yields a Burgers' type asymptotic model, not integrable, which is sometimes referred to as the Zabolotskaya-Kokhlov equation [82]. However, explicit solutions can be given; they describe the coalescence of $n$ quasi-one-dimensional wave fronts, all propagating in the same direction [83]. A complete mathematical proof of the convergence of this asymptotic has been recently given $[26,84]$. Here, the direction of the transverse modulation is perpendicular to the plane yielded by the applied field and the propagation direction. A modulation along the other transverse direction also arises; it involves a small drift of the wave. The corresponding asymptotic model is derived by means of the change of scales

$$
\begin{aligned}
\xi & =\varepsilon(x-V t), & & \eta=\varepsilon^{2}(y-U t), \\
\zeta & =\varepsilon^{2} z, & \tau & =\varepsilon^{3} t .
\end{aligned}
$$

Despite the presence of a transverse velocity $U$, it remains in the general frame defined by figure 9 in section 4.5. Indeed, the transverse variables $\eta$ and $\zeta$ are slow with regard to the 


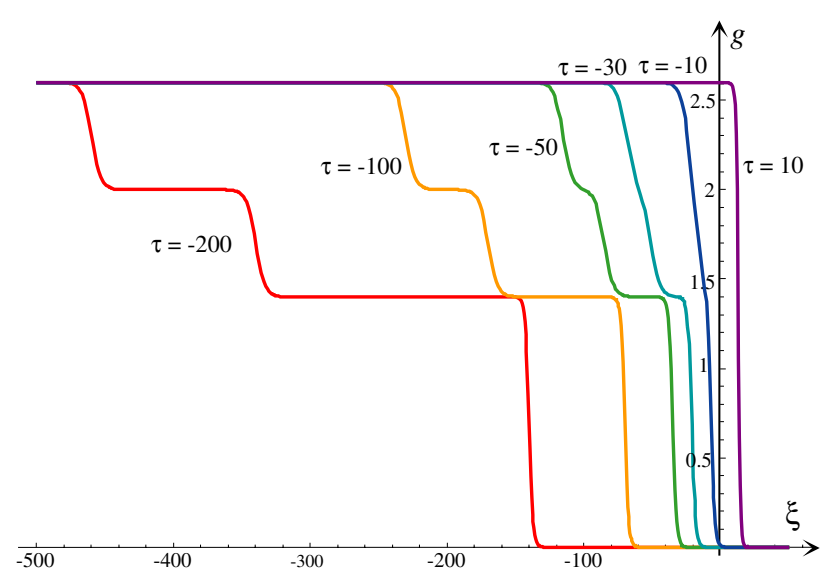

Figure 14. Coalescence of shock waves described by the Burgers' equation. Three successive shock fronts are seen on the leftmost curve, at $\tau=-200$. They coalesce to yield finally a single front on the rightmost curve, at $\tau=+10$.

longitudinal variable $\xi$, and $U$ is a small correction of the velocity $V$. It is remarkable that the transverse modulation possesses such a drift velocity, whose direction is fixed by that of the applied magnetic field. The value of the perturbative parameter $\varepsilon$ is determined by the length of the solitary wave. It is seen that the Burgers' model (112) is obtained for propagation times much smaller than the KdV solitons (109): of order $1 / \varepsilon^{2}$ instead of $1 / \varepsilon^{3}$. The required amplitudes also differ, because they are adjusted so that the nonlinear effect arises at the expected time. A weak damping assumption of the form

$$
\sigma=\varepsilon \tilde{\sigma}
$$

allows us to describe the simultaneous arising of both effects. Using both the tridimensional expansion (116) and the weak damping assumption (117), we obtain a rather complete treatment of the problem [85]. The magnetization writes

$$
\vec{M}=\vec{m}+\varepsilon^{2} g \vec{m}_{1}+O\left(\varepsilon^{3}\right),
$$

and the magnetic field $\vec{H}$ is expanded in an analogous way. The lowest order in the perturbation theory, $\varepsilon^{2}$, fixes the polarizations $\vec{m}_{1}$ and $\vec{h}_{1}$. The following order $\varepsilon^{3}$ fixes the longitudinal velocity to $V=\sqrt{\left(\alpha+\sin ^{2} \varphi\right) /(1+\alpha)}$, characterizing the considered propagation mode, together with the transverse velocity $U=V\left(1-1 / V^{2}\right) \tan \varphi$. At order $\varepsilon^{4}$, we obtain the following evolution equation for the amplitude $g$ :

$$
\left(g_{\tau}+P g g_{\xi}+Q g_{\xi \xi \xi}+R g_{\xi \xi}\right)_{\xi}+S g_{\eta \eta}+T g_{\zeta \zeta}=0 .
$$

This equation is of KP type, with an additional nonconservative term $R g_{\xi \xi}$. The latter vanishes if the damping is negligible, i.e., formally, if the hypothesis (117) can be replaced by $\sigma \in o(\varepsilon)$. If in addition the problem is reduced to $(2+1)$ dimensions, assuming that there is a transverse modulation in one direction ( $y, z$ or other) only, equation (119) reduces exactly to KP. The explicit computation of the coefficients allows us to determine their sign: $Q$ is negative, and $S$ and $T$ positive. Equation (119) is thus KP I that admits algebraically decreasing bidimensional localized solutions, called lumps [51]. An example of such a solution is drawn in figure 15 .

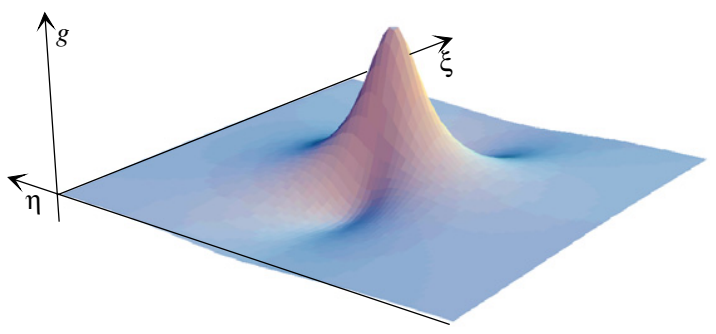

Figure 15. Solution lump of the Kadomtsev-Petviashvili equation. It is a stable solitary wave localized in two dimensions, which decays algebraically in all directions.

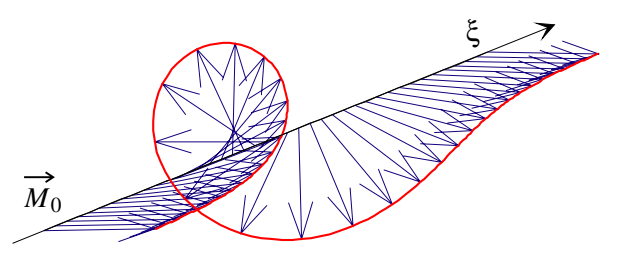

Figure 16. The structure of the solitons of the 'relativistic domain walls' type. The magnetization vector $\vec{M}_{0}$ rotates for one complete turn around the $\xi$-axis, and propagates in this direction.

\subsection{Relativistic domain walls and solitons}

Beside the spin waves, a type of strongly nonlinear waves exists in ferromagnetic media, called relativistic domain walls. Explicit exact solutions of the Maxwell-Landau equations have been obtained [86]. These waves have the following structure: the magnetization density vector rotates around the propagation direction, its tip describing a helical curve, that propagates along its axis, cf figure 16. This type of structure is close to that of the domain walls. Further, their analytic expressions are rather close to those which have been given by Landau for the latter [87]. A multiscale approach of 'long-wave' type allows us to find an alternative description for them. The energy of the structures depends mainly on the magnitude of the magnetization gradient and hence increases as the size of the structure decreases. The long-wave approximation is thus in fact a weak amplitude approximation, although the zero-order term in the expansion itself varies. Nakata showed that, in this limit, the evolution of this type of wave is governed by the $\mathrm{mKdV}$ equation and obtained soliton solutions corresponding to one turn of the magnetization around the propagation direction [88]. As an interpretation, let us consider two parallel domain walls, in which the magnetization rotates in the same direction: when they join together, there is some topological obstruction to their annihilation and their coupling yields a propagating structure. Corrections to this type of model have been given, describing the effect of damping [89], inhomogeneous exchange and anisotropy [90], the eventual presence of free charges (it leads to an additional damping) [91] and the antiferromagnetic character of the medium [92].

For a propagation parallel to the external magnetic field, the situation is strongly modified, a NLS equation is derived, in which the complex variable is $\psi=M_{0}^{y}+\mathrm{i} M_{0}^{z}$, where $\vec{M}_{0}$ is the leading term of the magnetization and 
$x$ is the propagation direction [18]. For a static field almost parallel to the polarization direction, and assuming that the inhomogeneous exchange interaction dominates, the asymptotic model satisfied by $\psi$ becomes a derivative NLStype equation [93].

The effect of transverse perturbations on these structures can be studied by means of a multiscale expansion, which leads to the integro-differential set of equations [94]

$$
\begin{aligned}
\theta_{\tau} & +\mu \theta_{\xi \xi \xi}+\frac{\mu}{2}\left(\theta_{\xi}\right)^{3} \\
= & -\gamma \theta_{\xi \xi}-\rho \Phi+\theta_{\xi} \int_{-\infty}^{\xi}\left(\rho \Psi-\gamma \theta_{\xi}^{2}\right) \mathrm{d} \xi^{\prime} \\
\Psi_{\xi} & =\Phi \theta_{\xi}-\left(\theta_{\eta}^{2}+\theta_{\zeta}^{2}\right) \\
\Phi_{\xi} & =-\Psi \theta_{\xi}+\theta_{\eta \eta}+\theta_{\zeta \zeta}
\end{aligned}
$$

where $\mu, \gamma$ and $\rho$ are real constants, which can be expressed as functions of the physical parameters. The stability of the solutions depends closely on the sign of the $\rho \mu$ product, which is always positive. $v=\theta_{\xi}$, where $\theta$ is the precession angle of the magnetization $\vec{M}_{0}$ around the propagation direction $x$, i.e.,

$$
\vec{M}_{0}=\left(\begin{array}{c}
m_{x} \\
m_{t} \cos \theta \\
m_{t} \sin \theta
\end{array}\right) .
$$

Equation (120) presents an obvious analogy with the following one, often called the modified Kadomtsev-Petviashvili (mKP) equation:

$$
\left(v_{\tau}+\mu v_{\xi \xi \xi}+\frac{3}{2} \mu v^{2} v_{\xi}\right)_{\xi}=-\rho v_{\eta \eta} .
$$

Recall that the mKP equation above, contrarily to the KP equation (79), is not integrable. It admits however line solitontype solutions, since, when it is reduced to one dimension, it yields the $\mathrm{mKdV}$ equation. Equation (120) admits exactly the same reduction and thus exactly the same line solitons. The stability of these solutions with respect to a longitudinal perturbation is essentially governed by the mKdV equation. It is thus the stability of the soliton. Regarding transverse perturbations, the situation radically differs. The stability properties of $\mathrm{mKP}$ are the same as those of KP [7]. Let us recall that if the $\mu \rho$ product is negative, the KP equation is called KP I, and its line solitons are unstable with respect to slow transverse perturbations. If $\mu \rho$ is positive, they are stable, and the equation is KP II. With regard to fast transverse oscillations, the stability results are inverted, and it is for KP I that the line soliton is stable with regard to these kinds of perturbations.

The stability of the line soliton of the system (120)(122) has been discussed numerically and analytically [94]: the results are inverted with respect to the case of $\mathrm{KP}$ or mKP. Because $\mu \rho$ is always positive, the line solitons are unstable under transverse perturbations. The interactions of line solitons, as well as a localized initial input, always give rise to wave breaking, as far as numerical computations have been run. This instability is related to the fact that the first of the conservation laws of $\mathrm{mKdV}$ is not satisfied by equations (120)(122). It gives rise to the emission of other solitary waves, which seem to belong to the propagation mode governed by

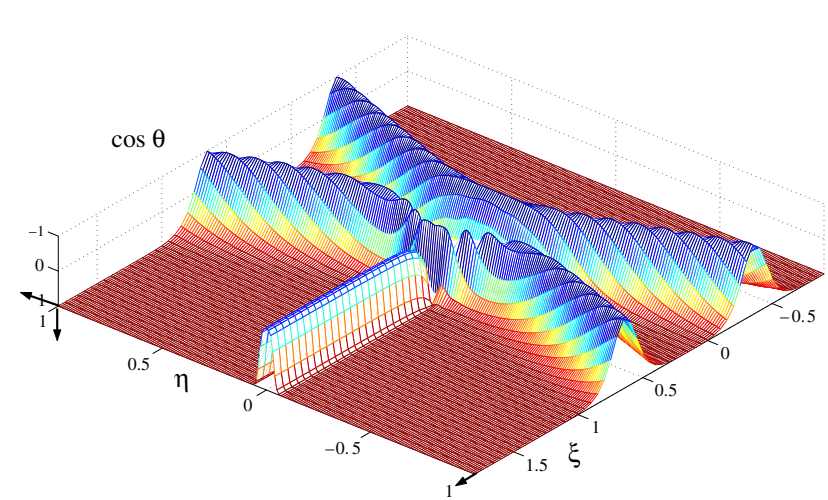

Figure 17. The instability described by equation (120) gives rise to the emission of a solitary wave of another type. Plot of $\cos \theta$, after an evolution time $\tau \simeq 6$, starting from initial data containing two line solitons linearly superposed. Instead of evolving to a smooth interaction pattern comparable to the one of figure 10, it gives rise to a narrow deformation in the direction $\xi$ of the propagation.

$\mathrm{KdV}$, cf figure 17. The direct numerical resolution of the Maxwell-Landau system showed the same kind of instability and wave emission, out of the long-wave approximation [95]. A perturbative approach of oscillations of these structures has shown in an analogous way spin wave emission [86].

\subsection{Interaction of long waves}

The above-mentioned emission of KdV-type waves by the transverse instability of the relativistic domain walls can be interpreted as an interaction between the two types of solitary waves. The interaction between two incident solitary waves, each of which belongs to one of the two modes, has been studied in the one-dimensional situation, i.e. assuming that the two incident waves are plane waves propagating in the same direction [31]. The equations describing the interaction are obtained by means of a multiscale expansion using the slow variables $\xi=\varepsilon x, \tau=\varepsilon t$.

The computation shows that, in order that the asymptotic model can describe the interaction, the magnetic field expansion must begin with

$$
\vec{H}=\vec{H}_{0}+\sqrt{\varepsilon} \vec{H}_{1}+O(\varepsilon) .
$$

This means that, in order for this interaction to occur, the wave amplitude must be much higher than what is necessary for the self-interactions studied above.

It is seen that the leading term of zero order is

$$
\vec{H}_{0}=\alpha\left(\begin{array}{c}
m \cos \varphi \\
m \sin \varphi \cos \theta \\
m \sin \varphi \sin \theta
\end{array}\right),
$$

i.e., that it describes a precession of the magnetization around the propagation axis $x$. The precession angle $\theta$ is a function of the variable $\xi-V_{0} \tau$, which accounts for a propagation at the velocity

$$
V_{0}=\sqrt{\frac{\alpha}{1+\alpha}} .
$$

This expression describes in first approximation the relativistic domain walls. Note that the angle $\varphi$ between the propagation 
direction and the uniform applied field is a constant geometrical parameter of the problem, while the angle $\theta$ is a dynamical variable. The following term $\vec{H}_{1}$ is split into a transverse component

$$
H_{1}^{\perp}=H_{1}^{y}+\mathrm{i} H_{1}^{z}=(\Psi+\mathrm{i} \Phi) \mathrm{e}^{\mathrm{i} \theta}
$$

and a longitudinal component $H_{1}^{x} \propto \Psi$. The function $\Psi$ accounts for the variations in the norm of the transverse component $H^{\perp}=H_{0}^{\perp}+\sqrt{\varepsilon} H_{1}^{\perp}+O(\varepsilon)$ of $\vec{H}$, i.e. for the variations of the angle $\varphi$ (recall that $\|\vec{M}\|$ is a constant). It is the propagation mode which under certain conditions can support KdV solitons, and which, by convenience, we will denote by this name. The other component, $\Phi$, accounts for the variations of the direction of $H_{\perp}$. It is in fact some correction to the precession angle $\theta$ of the magnetization around the propagation direction. In other words, $\Phi$ is a correction to the function $\theta$ which describes the relativistic domain wall; it contains all information regarding the retroaction of the $\mathrm{KdV}$ soliton onto this wave.

The system of equations which describes the interaction then becomes

$$
\left(\partial \xi^{2}-\lambda \partial_{T}^{2}\right) \Psi=F, \quad\left(\partial \xi^{2}-\partial_{T}^{2}\right) \Phi=G .
$$

The constant $\lambda$ is equal to $V_{0}^{2} / V_{1}^{2}$, where $V_{0}$ is the velocity of the relativistic domain wall-type waves, given by (127), and $V_{1}$ that of the waves of the $\mathrm{KdV}$ mode, i.e.,

$$
V_{1}=\sqrt{\frac{\alpha+\sin ^{2} \varphi}{1+\alpha}} .
$$

$T=V_{0} \tau$ is a normalized time variable, and the source terms $F$ and $G$ are functions of $\theta, \Psi, \Phi$ and their derivatives. Equations (129), written in an integral form, can be solved explicitly for particular initial data, for which $\mathrm{d} \theta / \mathrm{d} \xi$ approaches the distribution $2 \pi \delta(\xi-T)$. This expression of the precession angle $\theta$ can describe an incident $\mathrm{mKdV}$ soliton, in the limit where its size is very small with regard to all other lengths involved in the problem. The latter assumption is consistent with the rest of the theory and physically reasonable. We denote by $v$ the normalized velocity $v=$ $1 / \sqrt{\lambda}$, corresponding to the physical velocity $V_{1}$. If the incident $\mathrm{KdV}$ wave is $\Psi=g(\xi-v T)$, there is a transmitted wave $\Psi=\mathcal{T} g$, for $\xi>T$, i.e. after the interaction, cf figure 18 , and a reflected wave, which appears in the expression of $\Psi$ before the interaction point, adding a term to the expression of the incident wave:

$\Psi=g(\xi-v T)-\mathcal{R} g\left(-\frac{v-1}{v+1}(\xi+v T)\right), \quad$ for $\quad T>\xi$.

The reflection and transmission coefficients $\mathcal{R}$ and $\mathcal{T}$ have been expressed explicitly as functions of the normalized velocity $v$.

As happens frequently in soliton interactions, the $\mathrm{mKdV}$ soliton described by $\theta+\varepsilon \Phi$ undergoes a shift. The shift $\Delta$ is proportional to the integral of $g(\xi)$ over $\xi^{\prime}<0$, i.e., behind the relativistic domain wall. The sign of the shift $\Delta$ is thus the sign of the incident solitary wave of the $\mathrm{KdV}$ mode. If this sign is positive, this wave corresponds to an augmentation of the induction $\vec{B}$, and the relativistic domain wall is delayed. In the opposite case it is brought forward by the interaction.

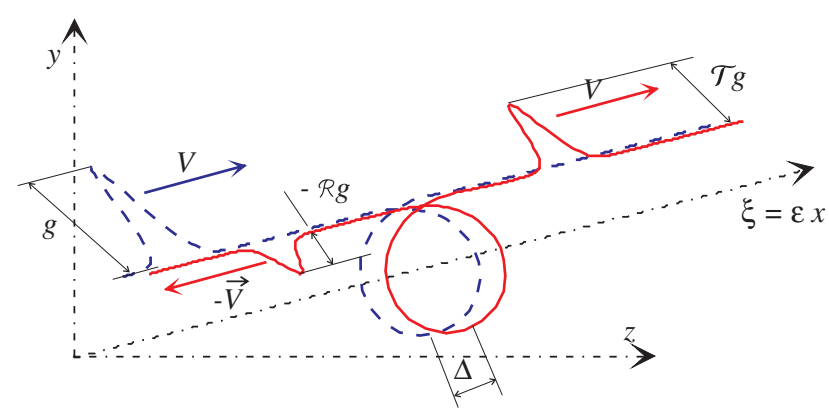

Figure 18. Schema of the interaction between a KdV soliton and a relativistic domain wall. Dotted line, the incident waves; solid line, the result of the interaction. The relativistic domain wall is shifted for $\Delta$, while the $\mathrm{KdV}$ soliton is partially transmitted and partially reflected. Only relative movement is depicted.

\subsection{Interactions between long waves and envelopes: shock wave emission}

An envelope soliton, or rather a localized packet of fast oscillating waves, can also interact with long solitary waves, consisting of a single oscillation, the duration of which has the same order of magnitude as the wave packet. This type of interaction can arise in the frame of soliton-type propagation of the wave packet, i.e. when the nonlinear effects have an order of magnitude comparable to that of the dispersion or of the diffraction. The model accounting for wave packet propagation and interaction with the solitary waves is thus a generalization of the NLS equation, which involves one or several auxiliary fields describing the solitary waves. The simplest situation yields the Davey-Stewartson (DS) system.

Another situation, which has been the focus of many mathematical studies, is the so-called nonlinear geometrical optics. Its mathematical importance is due to the fact that the convergence proofs for the multiscale expansion are much more accessible in this case. It is the situation where the nonlinear effect occurs at propagation distances of the same order of magnitude as the pulse length. This means that the nonlinear effect, which is much more intense than in the case of a NLS model, perturbs the transport at the group velocity. An alternative interpretation is to consider that the pulse length is very large, so that the dispersion is negligible.

Let us now look for the asymptotic description of an electromagnetic wave propagating in a ferromagnetic medium as described by the Maxwell-Landau model (102) and (103), corresponding to the nonlinear geometrical optics regime. We use an expansion analogous to (105), just as to derive the NLS model, but the profiles $\vec{M}_{n}^{p}$ are now functions of the slow variables [96]

$$
\tau=\varepsilon^{2} t, \quad \xi=\varepsilon^{2} x, \quad \eta=\varepsilon^{2} y, \quad \zeta=\varepsilon^{2} z
$$

In the special case where the static field is parallel to the propagation direction, the amplitude $g$ of the wave packet satisfies the nonlinear transport equation

$\mathrm{i} A f_{\tau}+\mathrm{i} A v f_{\xi}+B f|f|^{2}+C f \Phi+E v f \int_{-\infty}^{\tau} \partial_{\xi}|f|^{2}=0$ 
where $A, B, C$ and $E$ are real constants and $v$ is the group velocity. Equation (133) is solved directly, writing the amplitude $g$ in the form $g=r \mathrm{e}^{\mathrm{i} \theta}$. It is found that $r=r\left(\vec{\xi}^{\prime}\right)$ and

$\theta\left(\vec{\xi}^{\prime}, \tau\right)=\frac{B-E}{A} r^{2}\left(\vec{\xi}^{\prime}\right) \tau+\frac{C}{A} \int_{\tau_{1}}^{\tau} \Phi\left(\vec{\xi}^{\prime}, \hat{\tau}\right) \mathrm{d} \hat{\tau}$,

with $\xi^{\prime}=\xi-v \tau, \vec{\xi}^{\prime}=\left(\xi^{\prime}, \eta, \zeta\right)$. The first nonlinear effect is thus a phase modulation proportional to both the time and the squared wave amplitude. But this modulation presents a second term involving an auxiliary field $\Phi=\vec{H}_{2}^{0, x}$, which is a component of the term of order $\varepsilon^{2}$, corresponding to the zero harmonic, in the Fourier expansion of the magnetic field. In other words, $\Phi$ can describe an isolated oscillation, without a carrier. The equation describing its evolution is

$$
\left(V_{0}^{2} \partial_{\xi}^{2}+\partial_{\eta}^{2}+\partial_{\zeta}^{2}-\partial_{\tau}^{2}\right) \Phi=P \partial_{\tau}^{2} r^{2}
$$

where $P$ is a real constant and $V_{0}$ is the propagation velocity (127) of the relativistic domain walls, but it is also the velocity (130) of the KdV-type waves when they propagate parallel to the static field $(\varphi=0) . \Phi$ describes waves belonging to this mode, in three dimensions. The solution of equation (135) is the sum of a solution of the homogeneous equation and a particular solution $\Phi^{+}\left(\vec{\xi}^{\prime}\right)$ satisfying

$$
\left(\left(V_{0}^{2}-v^{2}\right) \partial_{\xi^{\prime}}^{2}+\partial_{\eta^{\prime}}^{2}+\partial_{\zeta^{\prime}}^{2}\right) \Phi^{+}=P v^{2} \partial_{\xi^{\prime}}^{2} r^{2}
$$

If the solitary waves propagate faster than the fast oscillating wave, $V_{0}>v$ and equation (136) is a Poisson equation, which admits the solution

$$
\Phi^{+}\left(\vec{\xi}_{1}\right)=\iiint_{\mathbb{R}^{3}} \frac{\rho(\vec{u}) \mathrm{d} \vec{u}}{\left\|\vec{\xi}_{1}-\vec{u}\right\|},
$$

with $\rho=P v^{2} \partial_{\xi}^{2} r^{2}, d=\sqrt{\left|V_{0}^{2}-v^{2}\right|}, \xi_{1}=\xi^{\prime} / d$ and $\vec{\xi}_{1}=$ $\left(\xi_{1}, \eta, \zeta\right)$. This solution is smooth even if the wave packet is very localized, and if the support of $r$ reduces to a point or to a line.

If in contrast the fast oscillating wave packet is the faster one, $V_{0}<v$, then equation (136) has the form of a wave equation. Let us choose the following particular expression of the wave intensity:

$$
r^{2}=a H\left(\xi^{\prime}\right) \delta(\eta)
$$

where $H$ is the Heaviside function and $\delta$ the Dirac distribution. This intensity corresponds to the front end of a long pulse, emitted though a long and narrow slot. The solitary wave $\Phi^{+}$ of it emitted by the wave packet is then

$$
\Phi^{+}=\frac{-a P v^{2}}{2 d^{2}}\left[\delta\left(\eta+\frac{\xi^{\prime}}{d}\right)+\delta\left(\eta-\frac{\xi^{\prime}}{d}\right)\right] .
$$

It is thus singular, concentrated on the two half-planes $\eta=$ $\pm \xi^{\prime} / d$. The localized pulse emits a solitary wave, which remains smooth as long as the pulse velocity is lower than that of the solitary waves, but becomes a shock wave in the inverse
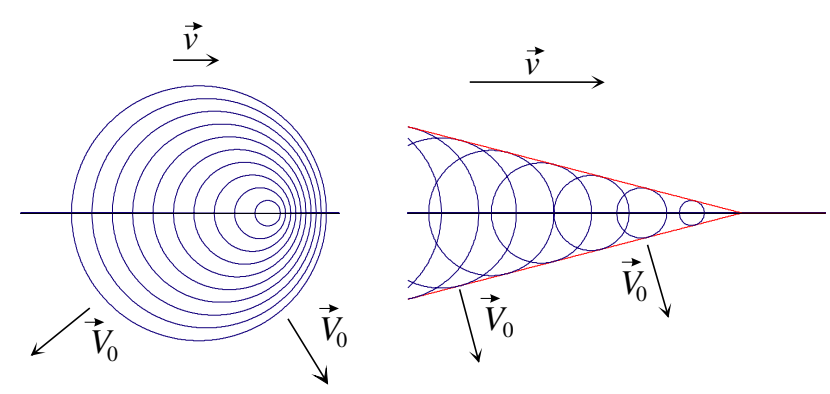

Figure 19. Schema showing the waves emitted by a source moving with a velocity $\vec{v}$. Left, their propagation velocity is larger than that of the source: the wave remains smooth. Right, the source propagates faster: the waves concentrate on a cone.

case, as shows the schema of figure 19. This phenomenon can be compared to the emission of an acoustic shock wave by a supersonic aircraft and to Tcherenkov emission. If the propagation direction is not parallel to the static field, the phenomenon is analogous, but more complicated. Indeed, the other solitary wave propagation mode, the relativistic domain walls, is also involved [96].

\section{An ab initio approach to nonlinear optics}

\subsection{Introduction}

When studying the propagation of electromagnetic solitons in ferromagnetic media, we have been able to avoid the use of 'nonlinear susceptibilities' and to derive the nonlinear propagation equations of NLS type directly starting from the Maxwell-Landau equations. From the theoretical point of view, the use of nonlinear susceptibilities is not totally satisfying, since it is the extension of an intrinsically linear notion to a frame which is not. A priori, such a generalization may be adequate for particular questions only. The use of these objects requires many precautions and can in some cases give erroneous results. Further, in some cases, the direct approach is much simpler: that is what happened for [77].

What is well defined is the nonlinear response function, initially introduced by Volterra [97, 98]. But it is a global description of the nonlinear propagation phenomenon that expresses what can be observed at the output of the setting as a function of the input, without any attempt to describe what happens inside. This notion may coincide with that of nonlinear susceptibility when the propagation distances are small. To write a nonlinear propagation equation for large propagation distances is a local approach, that radically differs from the global one. A priori, to mix both approaches, local and global, is likely not the most satisfying. That is why we propose a method which allows us to derive the macroscopic nonlinear propagation equation directly from the microscopic theory of quantum mechanics [99].

\subsection{Envelope solitons}

We use the density matrix formalism. The dynamics of each atom is described by a Hamiltonian $H_{0}$, to which we 
add a coupling term describing the interaction between the electric field $\vec{E}$ and the dipolar electric momentum of the atom, described by the operator $\vec{\mu}$. The Hamiltonian $H$ of the system decomposes as

$$
H=H_{0}-\vec{\mu} \cdot \vec{E}
$$

and the Schrödinger equation is

$$
\mathrm{i} \hbar \partial_{t} \rho=[H, \rho],
$$

where $\rho$ is the density matrix. The electric field satisfies the Maxwell equations, which reduce to

$$
\vec{\nabla}(\vec{\nabla} \cdot \vec{E})-\Delta \vec{E}=\frac{-1}{c^{2}} \partial_{t}^{2}(\vec{E}+4 \pi \vec{P})
$$

in the absence of magnetic effects. $c$ is the light velocity in vacuum, $\vec{\nabla}$ is the gradient operator and $\vec{P}$ is the polarization density, which is expressed with the help of the dipolar electric momentum $\vec{\mu}$ as

$$
\vec{P}=N \operatorname{tr}(\rho \vec{\mu}),
$$

with $N$ being the number of atoms by volume unit. Using these definitions, the system of equations (141) and (142) is called Maxwell-Bloch equations, although some authors prefer to keep this name for a reduction of the same system, cf [100], section 5.4.

For the sake of simplicity, we assume that $H_{0}$ is a nondegenerated two-level Hamiltonian,

$$
H_{0}=\hbar\left(\begin{array}{cc}
\omega_{a} & 0 \\
0 & \omega_{b}
\end{array}\right)
$$

and that the dipolar momentum of the stationary states is zero, which yields the following expression for the operator $\vec{\mu}$ :

$$
\mu_{s}=\left(\begin{array}{cc}
0 & \mu_{s} \\
\bar{\mu}_{s} & 0
\end{array}\right) \quad(s=x, y, z) .
$$

We apply the reductive perturbation method in expanding the electric field according to

$$
\vec{E}=\sum_{n \geqslant 1, p \in \mathbf{Z}} \varepsilon^{n} \mathrm{e}^{i p \varphi} \vec{E}_{n}^{p},
$$

where the profiles $\vec{E}_{n}^{p}$ are functions of the slow variables

$$
\tau=\varepsilon\left(t-\frac{z}{V}\right), \quad \zeta=\varepsilon^{2} z
$$

This expansion allows us to specify the meaning of the weak amplitude assumption. Here it is exactly the electrostatic energy of the atomic dipole in the wave field which is small with regard to the difference $\hbar \Omega=\hbar\left(\omega_{b}-\omega_{a}\right)$ between the energies of the atomic levels (we assume that $\omega_{b}>\omega_{a}$ ). We assume further that all the atoms are initially in the fundamental state and that few of them will be excited. The NLS equation is obtained as usual at the order $\varepsilon^{3}$ of the perturbative scheme; its nonlinear term involves the harmonics produced at order two. Two details, specific to the two-level model, must be noted: first, there is no zero harmonic, i.e. no optical rectification. Second, among the 'second harmonic' terms, the electric field $\vec{E}_{2}^{2}$ and the polarization density are zero; the diagonal terms $\rho_{2, a}^{2}, \rho_{2, b}^{2}$ of the density matrix only are nonzero. Thus, physically, there is no second harmonic, even produced in a non-resonant way. We write the NLS equation in the following form, usual in nonlinear optics:

$$
\mathrm{i} \partial_{\zeta} \mathcal{E}-\frac{1}{2} k_{2} \partial_{\tau}^{2} \mathcal{E}+\gamma \mathcal{E}|\mathcal{E}|^{2}=0
$$

where $\mathcal{E}$ is a component of the electric field amplitude, defined by $\vec{E}_{1}^{1}=\mathcal{E} \vec{u}$, where $\vec{u}$ is a unit vector. The coefficient of the dispersive term $-k_{2} / 2$ coincides exactly with the commonly admitted value: $k_{2}$ is the second derivative $\mathrm{d}^{2} k / \mathrm{d} \omega^{2}$ of the dispersion relation.

The coefficient of the nonlinear term can be compared to the results obtained using the nonlinear susceptibility tensor $\chi^{(3)}$ computed for the same two-level model, using, e.g., the density matrix formalism [100]. Note that our starting model is not isotropic. Let us first assume that the dipolar momentum operator $\vec{\mu}=(\mu, 0,0)$ is parallel to the $x$-axis, i.e. transverse. Then the nonlinear coefficient obtained through the multiscale expansion coincides exactly with that which is deduced from the nonlinear susceptibility $\chi^{(3)}$ :

$$
\gamma=\frac{-8 \pi N \mu^{2} \bar{\mu}^{2} \omega \Omega}{\hbar^{3} c \sqrt{\left(\omega^{2}-\Omega^{2}\right)^{3}\left(\omega_{2}^{2}-\omega^{2}\right)}} .
$$

In the case of a dipolar momentum that can be excited by a circular polarization only, $\mu_{x}=\mu, \mu_{y}=\mathrm{i} \mu$ and $\mu_{z}=0$, the multiscale expansion, adequately modified, shows that the nonlinear coefficient $\gamma$ is zero, while the use of nonlinear susceptibilities $\chi^{(3)}$ yields an erroneous result. The error seems to be due to the fact that, in the latter formalism, the effect of the circular polarization is obtained by splitting it into linear polarizations. But such a splitting uses the linearity and cannot give a correct result for a nonlinear phenomenon.

We can also assume that the charge oscillations can be excited in a fixed direction only that makes an angle $\alpha$ with the transverse plane $x y$, which is accounted for by $\vec{\mu}=(\mu \cos \alpha, 0, \mu \sin \alpha)$. For this simple model of an anisotropic situation, the use of the susceptibilities is not fully consistent and cannot give a correct expression of the nonlinear coefficient $\gamma$, while the above approach solves the problem.

Although this study is restricted to an academic problem, that of a non-degenerate two-level atom, it will be possible to generalize this approach to more realistic situations.

\subsection{A mKdV model for few-cycle pulses}

The recent progress in the development of solid-state modelocked lasers has resulted in the generation of two-cycle optical pulses [101-103]. The slowly varying envelope approximation (SVEA), under which the NLS model is derived, is not valid for few-cycle pulses. Several authors have attempted to describe the dynamics of such pulses by means of extensions of the SVEA [104, 105]. We propose here a radically different approach.

Leaving completely the concept of the slow envelope, the reductive perturbation method allows us to derive an approximate model able to describe few-cycle soliton propagation in a Kerr medium [106]. We consider the coupling between identical two-level atoms and the electric field described by the Maxwell-Bloch equations, i.e. equations (140)-(145) in the previous section. For the sake 
of simplicity, we assume a plane wave propagating along the $z$-axis and linearly polarized along the direction of the atomic dipolar electric momentum $\vec{\mu}$, i.e. $\vec{\mu}=\mu \vec{e}_{x}$ and $\vec{E}=E \vec{e}_{x}$, where $\vec{e}_{x}$ denotes the unitary vector along the $x$-axis.

Relaxation can be taken into account phenomenologically by modifying the Schrödinger equation (141) into

$$
\mathrm{i} \hbar \partial_{t} \rho=[H, \rho]+\mathrm{i} \hbar\left(\begin{array}{cc}
\rho_{b} / \tau_{b} & -\rho_{t} / \tau_{t} \\
-\rho_{t}^{*} / \tau_{t} & -\rho_{b} / \tau_{b}
\end{array}\right),
$$

where $\tau_{b}$ and $\tau_{t}$ are the relaxation times for the population and for the coherence, respectively.

We consider the situation where the wave duration $t_{\mathrm{W}}$ is long with regard to the period $t_{r}=2 \pi / \Omega$, with $\Omega=$ $\omega_{b}-\omega_{a}$, corresponding to the resonance frequency of the two-level atoms. We assume that $t_{\mathrm{w}}$ is about one optical period, say about $1 \mathrm{fs}$. Thus, we assume that the resonance frequency $\Omega$ is large with regard to optical frequencies. In order to obtain soliton-type propagation, the nonlinearity must balance dispersion, thus the two effects must arise simultaneously in the propagation. This involves a small amplitude approximation. Further, we can speak of a soliton only if the pulse shape is maintained over a large propagation distance. Therefore, we use the long-wave approximation method as defined in section 2.1. The electric field $E$, the polarization density $P$ and the density matrix $\rho$ are expanded as power series of a small parameter $\varepsilon$ as

$$
E=\sum_{n \geqslant 1} \varepsilon^{n} E_{n}, \quad P=\sum_{n \geqslant 1} \varepsilon^{n} P_{n}, \quad \rho=\sum_{n \geqslant 0} \varepsilon^{n} \rho_{n},
$$

and we introduce the slow variables

$$
\tau=\varepsilon\left(t-\frac{z}{V}\right), \quad \zeta=\varepsilon^{3} z
$$

Further, since the physical values of the relaxation times $\tau_{b}$ and $\tau_{t}$ are in the picosecond range, or even slower, i.e. very large with regard to the pulse duration $t_{\mathrm{w}}$, we write $\tau_{j}=\hat{\tau}_{j} / \varepsilon^{2}$ for $j=b$ and $t$. We assume that, in the unperturbed state, all atoms are in their fundamental state $a$.

The order-by-order resolution of the perturbative scheme leads to the following nonlinear evolution equation:

$$
\partial_{\zeta} E_{1}=\frac{4 \pi N|\mu|^{2}}{n c \hbar \Omega^{3}} \partial_{\tau}^{3} E_{1}+\frac{8 \pi N|\mu|^{4}}{n c \hbar^{3} \Omega^{3}} \partial_{\tau}\left(E_{1}^{3}\right)
$$

where $n=c / V=\sqrt{1+8 \pi N|\mu|^{2} /(\hbar \Omega)}$. Equation (153) is the $\mathrm{mKdV}$ one, already encountered in section 6.3. It also appears in various branches of physics such as anharmonic lattices and Alfvén waves in collisionless plasma. It is completely integrable by means of the inverse scattering transform [107]. The $N$-soliton solution has been given by Hirota [108].

The solution of equation (153) depends on the relative sign between the nonlinear and dispersion terms. In the present case, corresponding to a focusing nonlinearity, the $\mathrm{mKdV}$ equation cannot be reduced to the $\mathrm{KdV}$ one in the real domain [34]. Although the two-soliton solution in the sense of the IST is very far in shape from a few-cycle pulse, it can be algebraically prolonged, using complex values of the soliton parameters, to an explicit solution called a 'breather',

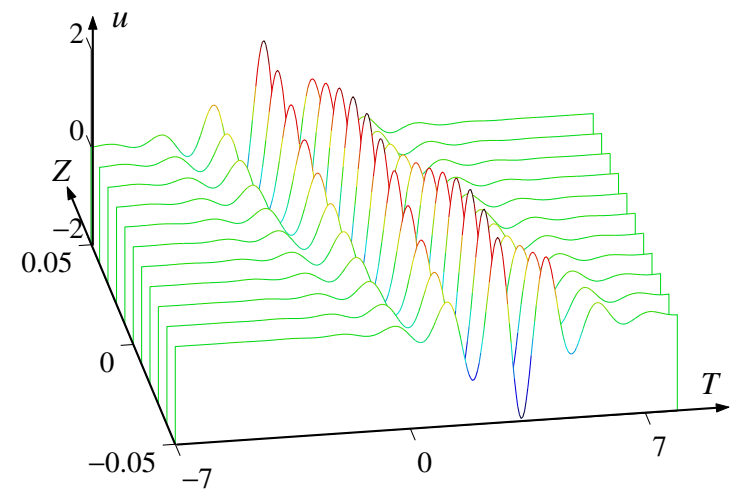

Figure 20. Breather solution of the $\mathrm{mKdV}$ equation, using dimensionless parameters.

(a)

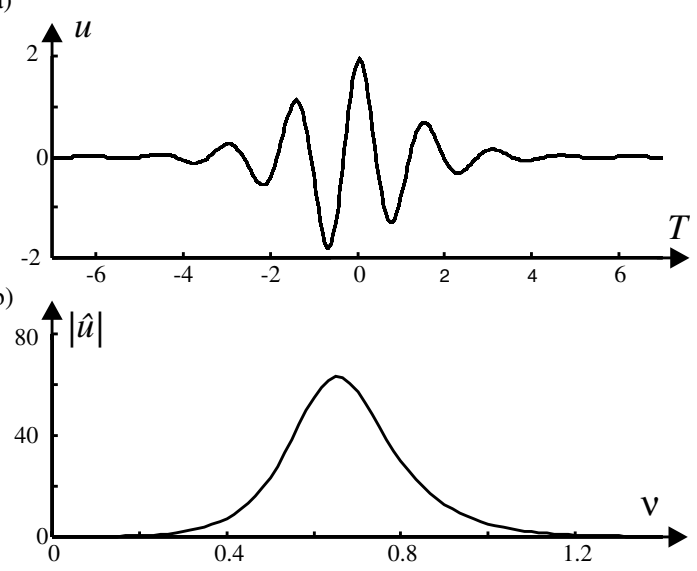

Figure 21. (a) Pulse profile and (b) spectrum of the second-order soliton solution of the mKdV equation of figure 20. It can model a few-cycle optical pulse. Dimensionless parameters are used.

which has typically the behaviour shown in figure 20 . The corresponding spectrum and pulse profile are given in figure 21. They are comparable to the experimental pulses shown in [103]. It can thus be thought that the two-cycle pulses produced experimentally could propagate as solitons in certain media, according to the $\mathrm{mKdV}$ model. Note that the $\mathrm{KdV}$ equation, and hence the $\mathrm{mKdV}$ one corresponding to a defocusing cubic nonlinearity, does not admit a breather solution. Indeed, the same prolongation procedure does not yield a regular solution.

Both the direct computation and the general theory [32] have shown that the coefficient of the dispersive term $\partial_{\tau}^{3} E_{1}$ in equation (153) is $(1 / 6) \mathrm{d}^{3} k / \mathrm{d} \omega^{3}$. A general expression of the nonlinear term is derived by considering the long-wave limit of the NLS equation (148); it is found that the nonlinear coefficient is $(6 \pi / n c) \chi^{(3)}$. Explicit computation shows that the general expression is valid in the particular case considered. Thus, equation (153) can be rewritten as

$$
\begin{aligned}
\partial_{\zeta} E_{1} & =\left.\frac{1}{6} \frac{\mathrm{d}^{3} k}{\mathrm{~d} \omega^{3}}\right|_{\omega=0} \partial_{\tau}^{3} E_{1} \\
& -\left.\frac{6 \pi}{n c} \chi_{x x x x}^{(3)}(\omega, \omega, \omega,-\omega)\right|_{\omega=0} \partial_{\tau}\left(E_{1}^{3}\right)
\end{aligned}
$$


It can be reasonably conjectured that equation (154), which has also been derived from quasi-adiabatic following approximation [109], will still hold in the more general case of an arbitrary number of atomic levels, when the inverse of the characteristic pulse duration is much smaller than any of the transition frequencies of the atoms.

\subsection{Short-wave approximation}

We consider again the two-level model described in section 7.3, but assuming now that the resonance frequency $\Omega$ of the atoms is below the optical frequencies [106]. Thus the characteristic pulse duration $t_{\mathrm{W}}$ is very small with regard to $t_{r}=2 \pi / \Omega$, and we use a short-wave approximation. We now introduce a small perturbative parameter $\varepsilon$, such that the resonance period is $t_{r}=\hat{t}_{r} / \varepsilon$, where $\hat{t}_{r}$ has the same order of magnitude as the pulse duration $t_{\mathrm{w}}$. The perturbative parameter $\varepsilon$ is thus about $t_{\mathrm{w}} / t_{r}$. Consequently, the Hamiltonian $H_{0}$ of the atom is replaced in the Schrödinger equation (150) by $\varepsilon \hat{H}_{0}$. We introduce a retarded time $\tau=t-z / V$ and a slow propagation variable $\zeta=\varepsilon z$. The electric field $E$ is expanded as $E=\sum_{n \geqslant 0} \varepsilon^{n} E_{n}$ and so on. The pulse duration $t_{\mathrm{w}}$ is still assumed to be about $1 \mathrm{fs}$, corresponding to an optical pulse of a few cycles, and the relaxation times $\tau_{b}$ and $\tau_{t}$ are very long with regard to $t_{\mathrm{w}}$. Since the above scaling uses $t_{\mathrm{w}}$ as zero-order reference time, it can be expressed by setting $\tau_{j}=\hat{\tau}_{j} / \varepsilon$ for $j=b$ and $t$. Although formally different, the weak damping assumption is physically the same as in the previous section. The scaling is equivalent to the standard short-wave approximation formalism developed, e.g., in [110-112].

The population inversion is $w=\rho_{0 b}-\rho_{0 a}$. It is shown by solving the perturbative scheme that $w$ and the leading term $E_{0}$ in the expansion of the electric field satisfy the set of equations

$$
\begin{array}{ll}
\partial_{\zeta} E_{0}=\frac{4 \mathrm{i} \pi \Omega N}{c} p, & \partial_{\tau} p=\frac{-\mathrm{i}|\mu|^{2}}{\hbar} E_{0} w, \\
\partial_{\tau} w=\frac{-4 \mathrm{i}}{\hbar} E_{0} p, &
\end{array}
$$

where

$$
p=\frac{-\mathrm{i}|\mu|^{2}}{\hbar} \int^{\tau} E_{0} w .
$$

Equations (155) coincide with the equations for self-induced transparency, although the physical situation is quite different: the characteristic frequency $1 / t_{\mathrm{w}}$ of the pulse is far above the resonance frequency $\Omega$, while the self-induced transparency occurs when the optical field oscillates at the frequency $\Omega$. The quantities $E$ and $w$ here describe the electric field and the population inversion themselves, and not amplitudes modulating a carrier with frequency $\Omega$. Note that $E$ and $w$ here are also real quantities, and not complex ones as in the case of the self-induced transparency. Further, $p$ is not the polarization density, but is proportional to its $\tau$-derivative. Another difference is the absence of a factor $1 / 2$ on the righthand side of equation (155).

System (155) can be reduced to

$$
\partial_{Z} \partial_{T} u=\sin u
$$

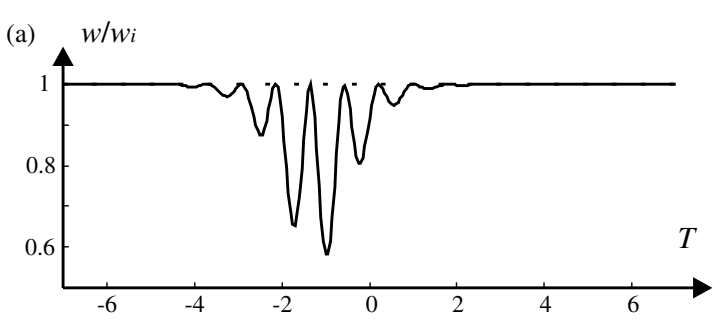

(b)
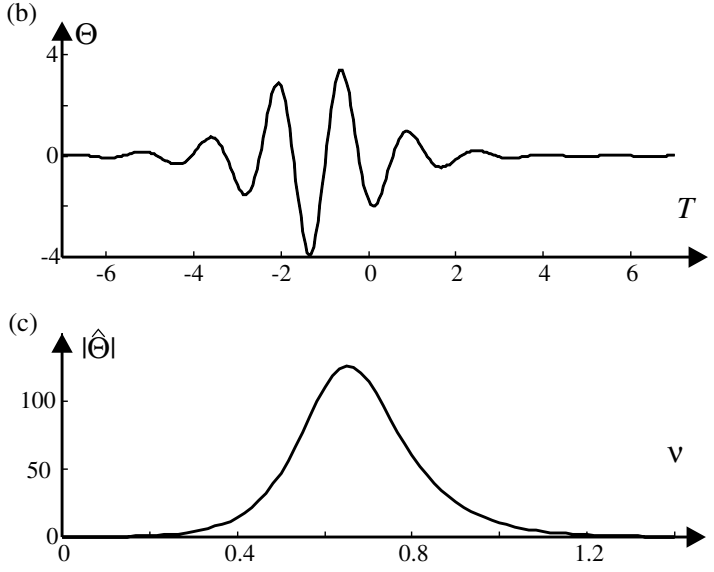

Figure 22. (a) Pulse profile, (b) population inversion and (c) spectrum of the breather solution of the sine-Gordon equation. It can model a few-cycle optical pulse. Dimensionless parameters are used.

in which

$$
\begin{aligned}
Z & =\frac{z}{\hat{L}}, & T & =\frac{1}{t_{\mathrm{w}}}\left(t-\frac{z}{c}\right), \\
E & =\frac{E_{r}}{2} \int^{Z} \sin u, & w & =w_{\mathrm{i}} \cos u .
\end{aligned}
$$

The electric field and propagation length scaling parameters are

$$
E_{r}=\frac{\hbar}{|\mu| t_{\mathrm{w}}}, \quad \hat{L}=\frac{\hbar c}{\Omega t_{\mathrm{w}} 4 \pi N|\mu|^{2} w_{\mathrm{i}}},
$$

in which the initial population inversion $w_{\mathrm{i}}$ and typical pulse duration $t_{\mathrm{w}}$ are given.

The sine-Gordon equation (157) is completely integrable [7]. An $N$-soliton solution can be found using either the IST or the Hirota method. As with the mKdV equation, it admits a breather solution, and hence is able to describe solitontype propagation of a pulse in the two-cycle regime. The pulse profile, with the corresponding population inversion and spectrum, is shown in figure 22. They are comparable with the experimental observation of [103]. The explicit breather solutions allow us to define envelope and carrier, group and phase velocities for a few-cycle soliton in an algebraic way, although these quantities a priori make sense within the SVEA only [113].

\section{Nonlinear optics of quadratic media}

\subsection{Propagation equations far from the phase matching}

Despite the reservations mentioned in the previous section, in a more general frame, the use of a phenomenological model 
of response functions, which are the nonlinear susceptibilities, allows us to determine the envelope propagation equations. Let us consider the evolution of the modulation of a short and localized optical pulse in a bulk medium, taking into account anisotropy and polarization [48]. The electric field $\vec{E}$ satisfies the Maxwell equations, which reduce to

$$
\vec{\nabla} \wedge(\vec{\nabla} \wedge \vec{E})=-\frac{1}{c^{2}} \partial_{t}^{2} \vec{D},
$$

where $\vec{D}$ is the electric induction, described by the following standard model [100]. It is written as the sum $\vec{D}=\vec{D}_{l}+\vec{P}_{n l}$ of a linear part $\vec{D}_{l}$, which satisfies

$$
\vec{D}_{l}=\chi^{(1) *} \vec{E}=\int_{-\infty}^{t} \mathrm{~d} t_{1} \chi^{(1)}\left(t-t_{1}\right): \vec{E}\left(t_{1}\right),
$$

and a nonlinear part $\vec{P}_{n l}$, corresponding to the nonlinear polarization, defined by

$$
\begin{aligned}
\vec{P}_{n l}= & \chi^{(2) *}(\vec{E}, \vec{E})+\chi^{(3) *}(\vec{E}, \vec{E}, \vec{E}), \\
= & \int_{-\infty}^{t} \mathrm{~d} t_{1} \int_{-\infty}^{t_{1}} \mathrm{~d} t_{2} \chi^{(2)}\left(t-t_{1}, t-t_{2}\right): \vec{E}\left(t_{1}\right) \vec{E}\left(t_{2}\right) \\
& +\int_{-\infty}^{t} \mathrm{~d} t_{1} \int_{-\infty}^{t_{1}} \mathrm{~d} t_{2} \int_{-\infty}^{t_{2}} \mathrm{~d} t_{3} \chi^{(3)} \\
& \times\left(t-t_{1}, t-t_{2}, t-t_{3}\right): \vec{E}\left(t_{1}\right) \vec{E}\left(t_{2}\right) \vec{E}\left(t_{3}\right) .
\end{aligned}
$$

The nonlinear susceptibilities $\chi^{(1)}, \chi^{(2)}$ and $\chi^{(3)}$ are tensors of rank 2, 3 and 4, respectively. We are interested in a situation where the second-order susceptibility $\chi^{(2)}$ is not zero. The materials satisfying this hypothesis, non-centrosymmetric, are in most cases isotropic or uniaxial regarding the linear optics described by $\chi^{(1)}$ [114]. We assume that, if the material is uniaxial, the propagation occurs parallel to its optical axis $z$, i.e. that the Fourier transform $\hat{\chi}^{(1)}$ of $\chi^{(1)}$ is

$$
\hat{\chi}^{(1)}=\left(\begin{array}{ccc}
n_{\mathrm{o}}^{2} & 0 & 0 \\
0 & n_{\mathrm{o}}^{2} & 0 \\
0 & 0 & n_{\mathrm{e}}^{2}
\end{array}\right)
$$

where $n_{\mathrm{o}}$ and $n_{\mathrm{e}}$ are, respectively, the ordinary and extraordinary indices of the medium. We retrieve the isotropic case by identifying $n_{\mathrm{e}}=n_{\mathrm{o}}$. We apply the reductive perturbation method, using the expansion (146) of the electric field $\vec{E}$ and the slow variables defined by (65) in section 4.1 , for a 'spherical' pulse. This leads to an asymptotic model of the DS type. The exact structure of this system depends on the symmetry properties of the nonlinear susceptibility tensors $\chi^{(2)}$ and $\chi^{(3)}$ and thus of the symmetry of the considered crystal. For the symmetry class $\overline{4} 2 m$, which contains many materials commonly used for their second-order nonlinear optical properties, in particular $\mathrm{KH}_{2} \mathrm{PO}_{4}$ (KDP), we obtain the system

$$
\begin{aligned}
& {\left[2 \mathrm{i} k \partial_{\zeta}+\beta \partial_{\xi}^{2}+\partial_{\eta}^{2}-k k^{\prime \prime} \partial_{\tau}^{2}\right] U+(\beta-1) \partial_{\xi} \partial_{\eta} V} \\
& =D_{1} U|U|^{2}+D_{2} U|V|^{2}+D_{3}(V)^{2} U^{*}+F \Phi V, \\
& {\left[2 i k \partial_{\zeta}+\partial_{\xi}^{2}+\beta \partial_{\eta}^{2}-k k^{\prime \prime} \partial_{\tau}^{2}\right] V+(\beta-1) \partial_{\xi} \partial_{\eta} U} \\
& =D_{1} V|V|^{2}+D_{2} V|U|^{2}+D_{3}(U)^{2} V^{*}+F \Phi U, \\
& {\left[\alpha\left(\partial_{\xi}^{2}+\partial_{\eta}^{2}\right)+\rho \partial_{\tau}^{2}\right] \Phi=Q\left(\partial_{\xi}^{2}+\partial_{\eta}^{2}\right)\left(U V^{*}+U^{*} V\right),}
\end{aligned}
$$

where $U=E_{1}^{1, x}$ and $V=E_{1}^{1, y}$ are the amplitudes of the two linear wave polarizations parallel to the crystallographic axes. The function $\Phi$ is a linear combination of the components $E_{2}^{0, x}$ and $E_{2}^{0, y}$ of the rectified field. The interaction constants can be expressed in terms of the components of the nonlinear susceptibility tensors $\hat{\chi}^{(2)}$ and $\hat{\chi}^{(3)}$. It is seen from the expressions that the coupling constant $Q$ accounts for optical rectification $(\omega-\omega \longrightarrow 0)$ and $F$ for the electro-optic effect $(\omega+0 \longrightarrow \omega) . \quad D_{2}$ and $D_{3}$ contain the combined effect of these two terms. $D_{2}$ also contains a term which accounts for the well known cascading effect $[39,115,116]$ : the generation of second harmonic $(\omega+\omega \longrightarrow 2 \omega)$, and back-conversion to the fundamental $(2 \omega-\omega \longrightarrow \omega)$. Finally, the three constants $D_{1}, D_{2}$ and $D_{3}$ obviously also contain components of the thirdorder nonlinear susceptibility $\hat{\chi}^{(3)}(\omega+\omega-\omega \longrightarrow \omega)$.

If the considered material belongs to the $3 m$ symmetry class, as does lithium niobate $\mathrm{LiNbO}_{3}$, the asymptotic model is even more complicated: both transverse components $E_{2}^{0, x}$ and $E_{2}^{0, y}$ of the rectified field intervene, and not only a single linear combination of these two quantities, which would correspond to a single polarization of the long wave. Further, the evolution equations of the two polarizations of the fundamental contain integro-differential nonlinear interaction terms [48].

\subsection{Interaction between polarizations}

The interaction of two polarizations in a one-dimensional medium has been studied by various authors [117, 118]. The system (165)-(167) describes the mutual interaction of two polarizations and their interaction with the rectified field, in $(3+1)$ dimensions. It can be reduced to $(1+1)$ dimensions, by assuming that the amplitudes $U, V$ and $\Phi$ depend on a single variable $X$ only, equal either to $\tau$ (temporal case) or to $(\xi \cos \theta+\eta \sin \theta)$ (spatial case). Equation (167) for the rectified field $\Phi$ is straightforwardly solved, and the system reduces to two coupled NLS equations:

$$
\begin{aligned}
& 2 \mathrm{i} k \partial_{\zeta} U+B_{1} \partial_{X}^{2} U+C \partial_{X}^{2} V \\
& \quad=D_{1} U|U|^{2}+D_{2}^{\prime} U|V|^{2}+D_{3}^{\prime} V^{2} U^{*} \\
& 2 \mathrm{i} k \partial_{\zeta} V+B_{2} \partial_{X}^{2} V+C \partial_{X}^{2} U \\
& \quad=D_{1} V|V|^{2}+D_{2}^{\prime} V|U|^{2}+D_{3}^{\prime} U^{2} V^{*}
\end{aligned}
$$

In the temporal case $X=\tau$, i.e. for a short pulse, whose section is large enough for the diffraction to be negligible, the coefficients are $B_{1}=B_{2}=-k k^{\prime \prime}, C=0, D_{j}^{\prime}=D_{j}$. In the spatial case, i.e. for a beam section which has an elongated shape, making an angle $\theta$ with the crystallographic axes (the beam can also be confined in a guide), the dispersion coefficients are more complicated:

$$
\begin{aligned}
& B_{1}=\beta \cos ^{2} \theta+\sin ^{2} \theta, \quad B_{2}=\cos ^{2} \theta+\beta \sin ^{2} \theta, \\
& C=(\beta-1) \sin \theta \cos \theta .
\end{aligned}
$$

We define constants $\alpha$ and $B$, accounting for the anisotropy at the frequencies zero and $\omega$ respectively, as

$$
\alpha=\frac{n_{\mathrm{o}}^{2}(0)}{n_{\mathrm{e}}^{2}(0)}, \quad \beta=\frac{n_{\mathrm{o}}^{2}}{n_{\mathrm{e}}^{2}} .
$$


The nonlinear coefficients are written as

$$
D_{j}^{\prime}=D_{j}+\frac{Q F}{\alpha}
$$

where $Q$ and $F$ are the same as above, involving $\hat{\chi}^{(2)}$ components. The term involving $\partial_{X}^{2} V$ in the evolution equation for $U(168)$ and the term involving $\partial_{X}^{2} U$ in that for $V$ vanish (i) in the temporal case, (ii) in the spatial case, when the medium is linearly isotropic $(\beta=1)$, and (iii) in the spatial case, if the angle $\theta$ between the direction of the modulation and the crystallographic axes is either $0, \pi / 4, \pi / 2$ or $3 \pi / 4$, eventually making use of an adequate change of variables.

There exist two completely integrable systems analogous to the system (168) and (169). Neither presents the terms involving $V^{2} U^{*}$ and $U^{2} V^{*}$ called 'four wave mixing'. The integrability of the system (168) and (169) requires thus at least that $D_{3}^{\prime}=0$. This condition can be satisfied if the interaction is not coherent. Otherwise, it is written, in the temporal case, as

$$
3 \hat{\chi}_{x y y x}^{(3)}(\omega, \omega,-\omega)=\frac{4}{n_{\mathrm{e}}^{2}(0)} \hat{\chi}_{x z y}^{(2)}(0, \omega) \hat{\chi}_{z x y}^{(2)}(\omega,-\omega) .
$$

One of these integrable systems, solved by Manakov [119], requires further that $B_{1}=B_{2}$ and $D_{1}=D_{2}^{\prime}$. The first of these conditions is satisfied in the isotropic spatial case and in the temporal case. The second one is written, in the temporal case, as

$$
\begin{aligned}
& 3\left(\hat{\chi}_{x x y y}^{(3)}(\omega, \omega,-\omega)+\hat{\chi}_{x y x y}^{(3)}(\omega, \omega,-\omega)-\hat{\chi}_{x x x x}^{(3)}(\omega, \omega,-\omega)\right) \\
& =4\left(\frac{1}{n_{\mathrm{e}}^{2}(2 \omega)} \hat{\chi}_{x z y}^{(2)}(2 \omega,-\omega) \hat{\chi}_{z x y}^{(2)}(\omega, \omega)\right. \\
& \left.\quad+\frac{1}{n_{\mathrm{e}}^{2}(0)} \hat{\chi}_{x z y}^{(2)}(0, \omega) \hat{\chi}_{z x y}^{(2)}(\omega,-\omega)\right) .
\end{aligned}
$$

Both integrability conditions (173) and (174) represent an equilibrium between components of the third-order susceptibility $\chi^{(3)}$ and contributions to the cascading $\chi^{(2)}$ : $\chi^{(2)}$. However it is rather unlikely that these conditions are satisfied by some real material. In the spatial case, the integrability conditions are analogous, but more complicated. The other integrability case, that of Zakharov and Schulman [120], requires in addition to other conditions that $B_{1}=-B_{2}$, which could be eventually realized for a spatio-temporal reduction, but not in the situations considered above.

\subsection{The Davey-Stewartson model}

The model (165)-(167), which describes the evolution of a tridimensional light pulse in a crystal with a nonzero quadratic nonlinear susceptibility $\chi^{(2)}$, is a generalization to $(3+1)$ dimensions and two polarizations of the DS system. We are looking for the possibility of a single polarization to propagate in $(2+1)$ dimensions. In the spatial bidimensional case, i.e. if there is no dependency with respect to the $\tau$ variable, equation (167) is solved straightforwardly and the two equations (165) and (166) reduce to two coupled twodimensional nonlinear Schrödinger-type equations, i.e. to a generalization of the 2D NLS equation (67), which takes into account two polarization components.

The spatio-temporal case in $(2+1)$ dimensions corresponds to the propagation of a short pulse in a planar guide. The model obtained when simply considering one of the two space variables of the tridimensional model only should give a correct account of the guided propagation, if the guide thickness is large enough, so that the effect of the guiding on the dispersion can be neglected. In this case, the system (165)-(167) can be reduced to the DS system (68) [121, 122]. However, the reduction is possible only if the light is polarized linearly, in a direction fixed with respect to both the direction of the modulation and the crystallographic axes. In order to specify this with respect to the $\overline{4} 2 m$ symmetry class, let us denote by $\Theta$ the angle between the direction of the polarization and the $x$-axis of the crystal, so that

$$
E_{1}^{1, x}=U=\varphi \cos \Theta, \quad E_{1}^{1, y}=V=\varphi \sin \Theta,
$$

where $\varphi$ is an amplitude to be determined. The unique transverse space variable $X$, which corresponds to the direction of the modulation, hence to the plane of the guide, must then be defined either by $X=\xi \cos \Theta+\eta \sin \Theta$ or by $X=-\xi \sin \Theta+\eta \cos \Theta$. The first case corresponds to a polarization direction parallel to the modulation direction, thus to the waveguide plane, and the second to a polarization perpendicular to the modulation. Further, the angle $\Theta$ can take the values $\pm \pi / 4$ only. When $\theta=0$ or $\pi / 2$, the rectified field $\Phi$ identically vanishes, and the asymptotic model is of 2D NLS-type. Making use of an adequate change of variables, the obtained system can be put into the reduced form (76), that I recall here, with a small change in the notations:

$$
\begin{gathered}
\mathrm{i} f_{T}+\varepsilon_{1} f_{X X}+f_{Y Y}+\varepsilon_{2} f|f|^{2}+v f \psi=0, \\
\psi_{X X}+\mu \psi_{Y Y}=|f|_{X X}^{2} .
\end{gathered}
$$

The variables $f, \psi, Y$ and $T$ are proportional to $\varphi, \Phi, \tau$ and $\zeta$, respectively.

The DS system (176) and (177) derived this way is integrable if the conditions (77) are satisfied, i.e. $\mu=-\varepsilon_{1}, v=$ $-2 \varepsilon_{2}$. The first of these two conditions writes

$$
\alpha k k^{\prime \prime}=\left(\frac{1}{v^{2}}-\frac{n_{0}^{2}(0)}{c^{2}}\right) B .
$$

If we leave aside the factors $\alpha$ and $B$, which describe a distortion due to the anisotropy, the condition (178) represents an equilibrium between the dispersion coefficient $k k^{\prime \prime}$ and a quantity that measures the difference between the group velocity $v$ of the wave and that $c / n_{\mathrm{o}}(0)$ of the solitary wave emitted through optical rectification. As the coefficient $k k^{\prime \prime}$ does, this quantity measures a dispersion. Equation (178) represents thus an equilibrium between the dispersion due to the rectified wave on the one hand and the proper dispersion of the wave on the other hand. Using the complete symmetry property of the nonlinear susceptibility tensors [100], and considering the symmetry classes of crystals $\overline{4} 2 m$ and $\overline{4} 3 m$, the second integrability condition is

$$
\begin{aligned}
& 3\left[\hat{\chi}_{x x x x}^{(3)}(\omega, \omega,-\omega)+\hat{\chi}_{x x y y}^{(3)}(\omega, \omega,-\omega)\right. \\
& \left.\quad+\hat{\chi}_{x y x y}^{(3)}(\omega, \omega,-\omega)+\hat{\chi}_{x y y x}^{(3)}(\omega, \omega,-\omega)\right] \\
& =\frac{4}{n_{\mathrm{e}}^{2}(0)}\left(\hat{\chi}_{x z y}^{(2)}(0, \omega)\right)^{2}+\frac{4}{n_{\mathrm{e}}^{2}(2 \omega)}\left(\hat{\chi}_{x z y}^{(2)}(2 \omega,-\omega)\right)^{2} .
\end{aligned}
$$

It expresses an equilibrium between the third-order Kerr effect and that induced by the cascaded second-order nonlinearities. 
The existence of localized solutions depends on the sign conditions. The lump solitons exist when $\varepsilon_{1}$ and $\varepsilon_{2}$ have opposite signs. Taking the explicit expressions of these coefficients into account, and using the complete symmetry property, it is found that $-\varepsilon_{1} \varepsilon_{2}$ is a square. The lump solitons thus exist in all integrable situations. The generalization of this kind of solution to non-integrable situations remains to be studied. Localized solitons, exponentially decreasing, exist for DS I, i.e. if $\varepsilon_{1}=+1$. This corresponds to the case of an anomalous dispersion: $k^{\prime \prime}<0$. Using the condition (178), this implies that the group velocity of the pulse must be higher than the velocity of the solitary long waves,

$$
v>\frac{c}{n_{\mathrm{o}}(0)} .
$$

The system DS I is of elliptic-hyperbolic type. The first equation is elliptic for an anomalous dispersion. The second one is hyperbolic when the condition (180) is satisfied. We can obtain numerical values from published experimental data [123]. For KDP, the velocities take the values

$v \simeq 1.95 \times 10^{8} \mathrm{~m} \mathrm{~s}^{-1} \quad$ and $\quad \frac{c}{n(0)} \simeq 0.65 \times 10^{8} \mathrm{~m} \mathrm{~s}^{-1}$.

For lithium niobate, we obtain

$v \simeq 1.2 \times 10^{8} \mathrm{~m} \mathrm{~s}^{-1} \quad$ and $\quad \frac{c}{n(0)} \simeq 0.5 \times 10^{8} \mathrm{~m} \mathrm{~s}^{-1}$.

For these materials, the second equation of the DS model is thus hyperbolic. This remains true if the integrability conditions (178) and (179) are not satisfied. The interaction between the wave packet and solitary long waves described by the DS system can be resonant, if an adequate matching of the wave velocities is satisfied. It then can lead to the splitting up of the wave packet into two or four parts; it can also transform a focusing situation into a defocusing one and vice versa $[121,124]$.

\subsection{Davey-Stewartson model for ferromagnets}

The (3+1)-dimensional evolution of an electromagnetic wave packet in a ferromagnetic medium, taking into account dispersion, diffraction and nonlinearity, is also described by a DS-type system. In the general case, two auxiliary fields describing the two propagation modes of solitary waves must be taken into account. However, if the propagation direction is parallel to the static field, the KdV-type waves only are involved [49]. If, further, the damping is neglected, the solitary wave field is assumed to vanish at infinity, and the problem is restricted to two variables; the evolution of the pulse shape is governed by the DS system (176) and (177) where $T, X, Y$, are slow variables proportional to $t, y$ and $x-v t$, respectively, $f$ is the amplitude of the fast oscillating pulse and $\psi$ that of the solitary long wave. The properties of the system DS (176) and (177), the general non-integrable case included, depend mainly on the sign of $\epsilon_{1}$ and $\mu$ [125]. The following classes

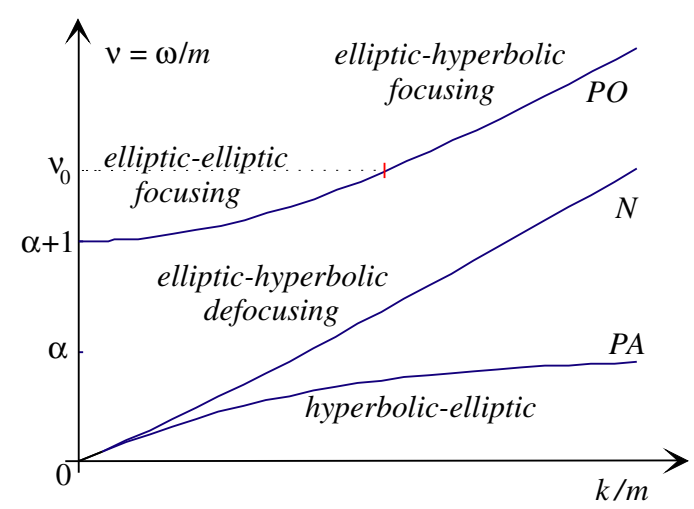

Figure 23. Plot of the dispersion relation of electromagnetic waves in ferromagnets, showing on each branch the class of the DS-type model which governs the transverse evolution of the wave packet.

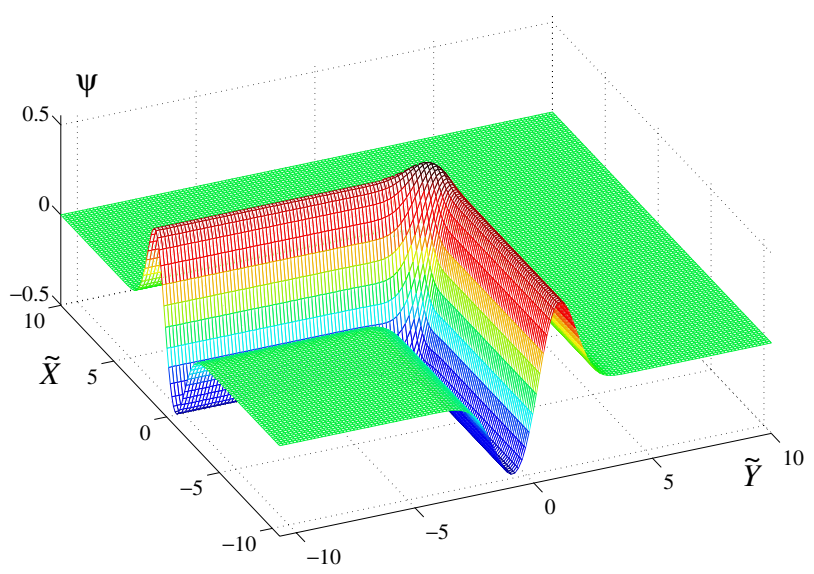

Figure 24. Shape of the emitted solitary wave $\psi$, described by the elliptic-hyperbolic DS system for waves with negative helicity ( $N$ in figure 23). The pulse is located close to the centre of the figure and propagates towards the upper right edge.

are distinguished:

\begin{tabular}{c|c}
$\left(\epsilon_{1}, \operatorname{sgn}(\mu)\right)$ & Type \\
\hline$(1,1)$ & Elliptic-elliptic \\
$(1,-1)$ & Elliptic-hyperbolic \\
$(-1,1)$ & Hyperbolic-elliptic \\
$(-1,-1)$ & Hyperbolic-hyperbolic
\end{tabular}

The physical situations can be classified according to these criterions; the result is summarized in figure 23. Equation (177) is elliptic when the velocity of the solitary waves is greater than the group velocity of the pulse. It is, in the frame of the approximation of nonlinear geometrical optics type discussed in section 6.5, the situation where equation (135) is a Poisson equation. The interaction with the solitary waves does not modify qualitatively the pulse behaviour, with respect to a (2+1)-dimensional NLS-type model. If in contrast the fast oscillating wave travels faster than the solitary waves, equation (177) is hyperbolic, $\mu=-s^{2}$. Then, there is a solitary wave emission concentrated in two preferential directions, as shown in figure 24 . This presents a strong analogy with the results of the long-wave analysis presented in section 6.5. If there is no incident solitary wave, as in the elliptic case, the 


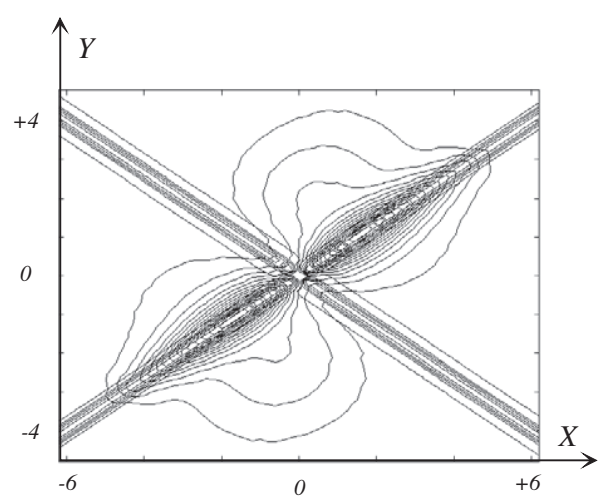

Figure 25. Elliptic-hyperbolic DS model for the wave with negative helicity ( $N$ in figure 23): splitting of a single pulse into two. Contour plot of both the high-frequency pulse and the long waves interacting together. The oblique lines forming a large cross shape represent the long waves; the pulse is split into two roughly elliptic parts.

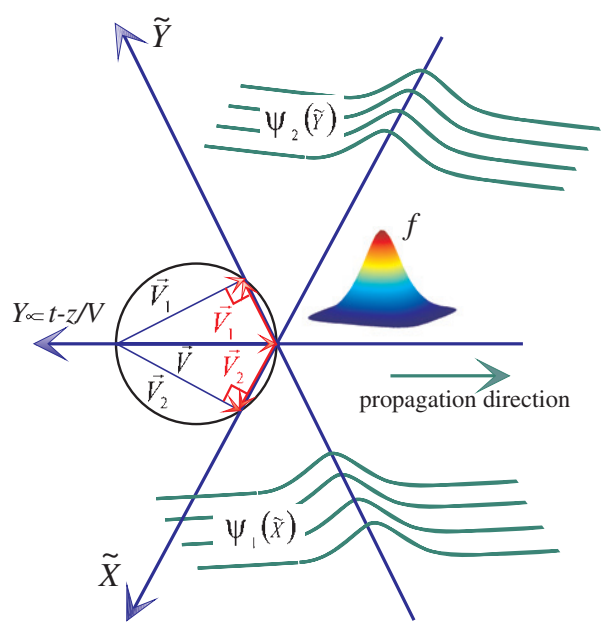

Figure 26. Schema of the interaction between solitary waves and a fast oscillating wave packet described by the elliptic-hyperbolic DS model. $\psi_{1}$ and $\psi_{2}$ are the incident solitary waves, with velocities $\vec{V}_{1}$ and $\vec{V}_{2}$, and $f$ the wave packet envelope, with the group velocity $\vec{V}$.

pulse behaviour is qualitatively the same as if no interaction occurs. In contrast, in the presence of adequately matched incident solitary waves, remarkable effects can be observed: the pulse can be split into several parts, cf figure 25, the dispersion and the diffraction can be stopped even if selfphase modulation was defocusing or, conversely, the pulse can be stabilized and the collapse avoided, despite the fact that the self-phase modulation alone would have led necessarily to it $[126,127]$ (see the next section). The resonance condition is described in figure 26 . The two incident solitary waves are line solitons, travelling with velocities $\vec{V}_{1}$ and $\vec{V}_{2}$. The tips of velocity vectors $\vec{V}_{1}$ and $\vec{V}_{2}$ lie on the circle whose diameter goes from the origin to the tip of the vector $\vec{V}$, the velocity of the optical pulse. Therefore, in the frame moving at the group velocity $\vec{V}$ of the optical pulse, the corresponding relative velocities $\vec{V}_{1}^{\prime}$ and $\vec{V}_{2}^{\prime}$ are perpendicular to $\vec{V}_{1}$ and $\vec{V}_{2}$, and hence parallel to the corresponding characteristic coordinates $\tilde{X}$ and $\tilde{Y}$, so that the plane waves are stationary in this frame [49].
This way, the duration of the interaction is very long, and it can be efficient even with a weak nonlinear effect. That is why these solitary waves, whose amplitude remains very small with regard to that of the pulse since they are terms of order $\varepsilon^{2}$, can have an important effect on it.

\subsection{Spatio-temporal optical pulse control using microwaves}

It is well known that optical solitons in Kerr media are unstable in more than $(1+1)$ dimensions. Saturating Kerr nonlinearities or cascaded second-order ones have been considered to stabilize multi-dimensional pulses, an issue which is very important for applications in optical telecommunications and integrated optics. Although many advances have been made (see the review in [128]), the experimental realization of stable spatio-temporal solitons is still a challenge. The interaction with microwaves described by the DS model offers an alternative way of producing such structures and shows that they are robust enough, so that they are promising for experiment and applications.

As seen above, dromion formation requires nonzero boundary values at infinity for the auxiliary field, which describes the rectified field, and this mathematical feature can been interpreted as an interaction between the pulse and solitary waves, whose length is comparable to the size of the pulse. One condition is that the DS system is of elliptichyperbolic type, i.e. that the solitary waves travel slower than the pulse. We have seen that this is the case in KDP and lithium niobate, as typical examples. Thus, pulse control through the solitary waves should be possible [124]. However, dromion formation requires a solitary wave with a single oscillation, which is even not symmetric when the sign of the electric field is changed. Since it is quite difficult to be realized in the microwave range, the interaction of a $(2+1)$ dimensional optical pulse with plane waves at microwave frequency has been considered [127]. This situation is related to the formation of solitons in periodic potentials [129, 130].

An efficient interaction requires a velocity matching, as shown in figure 26.

We assume that the second equation (177) in the DS system is hyperbolic, i.e. that the speed of the microwaves in the medium is smaller than the speed of light and that the self-phase modulation is focusing both spatially and temporally. As written above, the interaction can yield a soliton-type propagation, at input powers below the threshold for self-focusing. We show below that this stabilization can be obtained using sinusoidal input microwaves, instead of one-hump solitary waves, which renders the phenomenon achievable experimentally.

The DS system (176) and (177) is solved numerically using the scheme given in [131]. It uses the characteristic coordinates of the hyperbolic differential operator of equation (177): $\tilde{X}=Y-s X$ and $\tilde{Y}=Y+s X$. We consider a given initial condition $\varphi(T=0, \tilde{X}, \tilde{Y})=\varphi_{0}(\tilde{X}, \tilde{Y})$ and boundary data

$$
\begin{aligned}
& \psi_{1}(\tilde{X}, T)=\lim _{\tilde{Y} \longrightarrow-\infty} \psi(\tilde{X}, \tilde{Y}, T), \\
& \psi_{2}(\tilde{Y}, T)=\lim _{\tilde{X} \longrightarrow-\infty} \psi(\tilde{X}, \tilde{Y}, T) .
\end{aligned}
$$




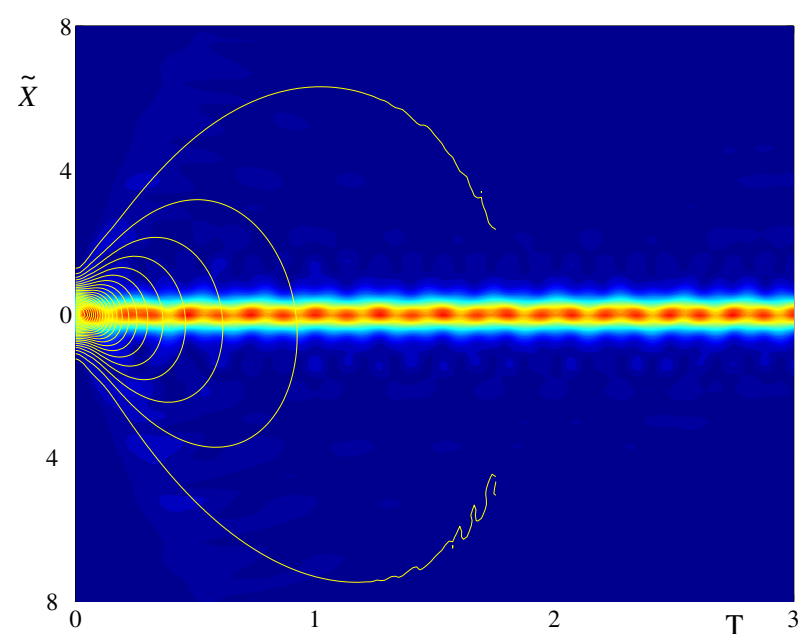

Figure 27. Stabilization of a short localized pulse due to the resonant interaction with microwaves: density plot showing the pulse profile in the direction $\tilde{X}=\tilde{Y}$, i.e. $Y$, against the propagation parameter $T$. Yellow lines indicate the pulse diffraction-dispersion in the absence of input microwaves.

$\psi_{1}$ and $\psi_{2}$ can give account of the two incident plane waves, propagating in such a direction that the interaction is 'resonant'. We consider sinusoidal waves with amplitudes $A_{1}, A_{2}$, wave numbers $k_{\tilde{X}}, k_{\tilde{Y}}$ and velocities $V_{\tilde{X}}, V_{\tilde{Y}}$, respectively. The initial input for the light pulse is a Gaussian. For certain values of the parameters, we observe the stabilization, as shown in figure 27.

The question of the robustness of the obtained 'driven soliton' when the input parameters are changed is discussed by evaluating the pulse radius after a given propagation distance $T$, when the parameters are varied.

The evolution depends on the phase of the microwave: stabilization occurs when the pulse is located at the zeros of the microwaves, while defocusing occurs when it is located at the maxima of amplitude. But the range in which stabilization occurs is very wide, more than a half period. A rather large velocity mismatch, up to $V_{\tilde{X}}, V_{\tilde{Y}} \simeq 4$ in normalized units, does not prevent stabilization. The pulse is driven at the velocity of the microwave pattern, as shown in figure 28. The range of the wave numbers $k_{\tilde{X}}, k_{\tilde{Y}}$ of the microwaves, of the initial pulse width and length $r_{\tilde{X}}$ and $r_{\tilde{Y}}$, of the initial pulse amplitude $A_{\varphi}$, of the parameters $\mu$ and $v$ which control dispersion and nonlinearities, for which the stability is obtained, is large enough to ensure the robustness of the pulse [127]. This will render possible its experimental realization, and opens the door to applications.

\section{Other applications}

Applications of the reductive perturbation method are numerous, and we do not pretend to give here an exhaustive list. However, we can mention a few important issues.

\subsection{Water waves}

The water wave theory has played an essential role in the development of both the soliton theory and the reductive

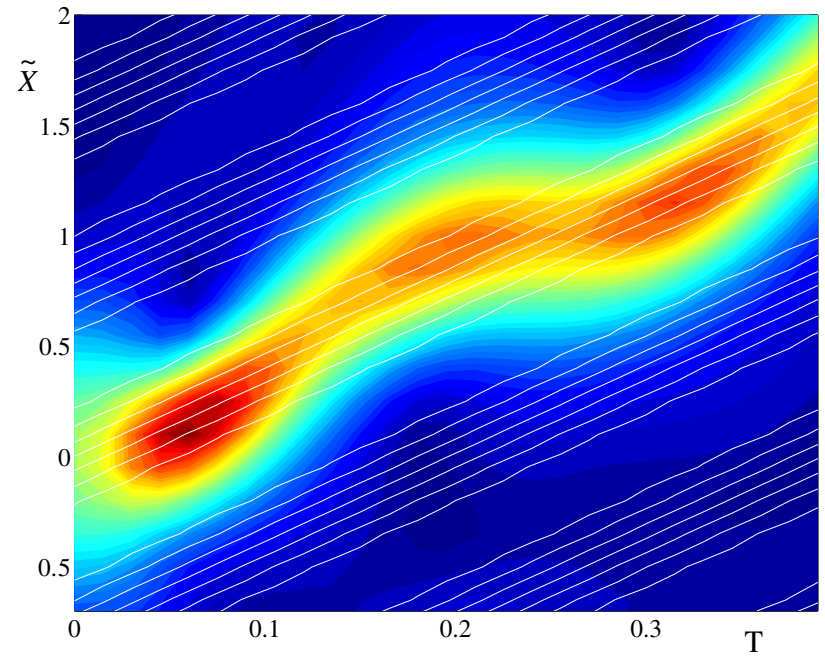

Figure 28. Evolution of the spatial profile of the pulse during the propagation, for a velocity mismatch $V_{\tilde{X}}=4, V_{\tilde{Y}}=0$. The white lines show the displacement of the input microwaves. Other parameters are as in figure 27.

perturbation method. Water waves have been already cited in many places throughout this tutorial.

First of all, the KdV soliton, with the pioneer works of Russell [1], Boussinesq [3], Korteweg and de Vries [4], is a water wave. More precisely, it is a short solitary wave in shallow water. As is known, $\mathrm{KdV}$ is integrable by means of the IST: the non-conservation of the Fourier modes and the conservation of the IST ones have been verified experimentally [132]. Envelope solitons are also concerned: the modulation instability, which corresponds to the evolution of a slowly modulated continuous wave into a train of solitons, was first found in the frame of water waves [79]; the DS system was originally derived by Davey and Stewartson in the same frame [46]. A review of integrable models in water waves can be found in [133].

Alternative models for water waves have been given, among which is the so-called BBM (Benjamin, Bona, Mahony) equation [134]. It is a kind of $\mathrm{KdV}$, in which one of the space derivatives in the dispersion term has been replaced by a time derivative. The derivation is perturbative but does not follow exactly the multiple scale scheme [135]. It has been shown that both models are equivalent at large times [136].

Waves at the interface separating two streams of immiscible inviscid fluids in relative motion (KelvinHelmholtz instability) have been described by means of a sineGordon equation [137]. (2+1) dimensional KdV and Burgers equations describing wave propagation in a fluid heated from below have been derived [139]. The surface tension can be taken into account, yielding the so-called Bénard-Marangoni system: asymptotic models of the same long-wave type have been derived [138]. Through the derivation of an envelope equation for the oscillating solutions to $\mathrm{KdV}$, it has been shown that no Benjamin-Feir instability occurs in this system, except at small Bond numbers [140].

The short-wave asymptotic dynamics has also been studied in water waves [112]. This approach has been applied 
to the Bénard system [141]; it has allowed surface wind to be taken into account [142] and to describe the wave profile in deep water [143].

\subsection{Dark solitons}

Dark solitons are dips in the envelope of a continuous monochromatic wave, which are stable due to the joint effect of dispersion and nonlinearity. An explicit dark-soliton solution to the NLS equation has been given [144], and the phenomenon has been observed in various domains of physics.

An alternative way of describing dark solitons is obtained by means of the reductive perturbation method. The wave behaviour is described by some envelope equation, typically of NLS type, and the continuous wave is some solution of constant amplitude to this equation. One seeks a solitary wave perturbation of this constant envelope, which leads to some $\mathrm{KdV}$-type model. Then, the soliton of $\mathrm{KdV}$ describes a dip in the envelope, which is the dark soliton. This approach allows us to account for dark solitons in non-integrable NLS-type equations. The third-order dispersion has been included, in $(1+1)$ [145] and $(2+1)$ dimensions [146]; in the latter case a KP model is derived. Nonlinearities other than the cubic Kerr one have been investigated [147, 148]. By means of a KdV Burgers' asymptotic model, it has been shown that the effect of Raman scattering results in a decay of the dark soliton [149, 150]. Introducing a cylindrical symmetry, ring dark solitons have been predicted [151]; they were experimentally obtained thereafter [152].

If the sign of the $\mathrm{KdV}$ soliton is inverted, it represents some short pulse propagating on the top of the continuous wave, which is called an anti-dark soliton [146]. Interactions between dark and anti-dark solitons have been studied $[153,154]$.

\subsection{Bose-Einstein condensates}

Bose-Einstein condensates (BECs) also obey a NLS equation, often referred to as the Gross-Pitaevskii equation. The NLS equation does not arise in this context as the result of the slowly varying envelope approximation (which is fundamentally the perturbative reduction method, even if it is not explicit), as in nonlinear optics or water waves. It is the Schrödinger equation of quantum mechanics, with an extra cubic term, which accounts for the interaction between two atoms [155].

The derivation of dark soliton can be performed in such a system as described in the previous section [156]. Their interactions have been studied [157]. In two dimensions, dark lumps have been predicted by means of a KP equation [158]. A modulated continuous wave may also propagate on the BEC. A DS model has been derived in this case, showing dromion propagation on the condensate [159].

Under certain conditions, corresponding in particular to low density, the inter-atomic coupling may be stronger. The cubic nonlinear term is then replaced by a quintic one in the model equation [160]. Dark solitons have also been obtained in this case by means of the reductive perturbation method $[161,162]$.

\subsection{Wave stability}

In some situations, the stability of a wave train or pulse can be investigated by means of the reductive perturbation method. The aim of Kadomtsev and Petviashvili when deriving their equation was to study the stability of the KdV soliton against transverse perturbations [56]. The result was that stability depends on the sign of the coefficients of the KP equation. The stability analysis itself involves the introduction of further slow variables [7, 163]. It has been generalized to other situations, e.g. to the transverse stability of long [85] and short [164] electromagnetic solitons in ferromagnetic media.

A second example of application of the method to the study of instabilities is the case of the so-called modulational or Benjamin-Feir instability. It was first evidenced in water waves [79]; thereafter it was shown that it occurs in the general NLS model [165]. Hence, the modulational instability for a continuous wave may be investigated through the derivation of a NLS equation by means of the reductive perturbation method. The knowledge of the coefficients of the equation allows a straightforward conclusion. This approach has been applied to waves in ferromagnetic media [77], the BénardMarangoni problem [140] and short water waves [167]. This instability analysis has been generalized to non-conservative systems and is widely used in this frame. However this falls outside the scope of this tutorial.

\section{Conclusion}

The above paragraphs would have convinced the reader that reductive perturbation is a very powerful and efficient method of deriving relevant models in the study of nonlinear wave propagation, whatever the particular physical situation. The method can be further developed to account for different types of problems, mainly in three directions. The first is the inclusion of additional assumptions on the magnitude of some coefficients. A very simple example is given by the weak damping approximation seen in sections 6.2 and 7.3. The second is the short-wave approximation, an example of which has been seen in section 7.4. It is a growing field, which would deserve a long discussion by itself. The third is the accounting for waveguide conditions in the perturbative scheme. The first attempt in this direction concerned magnetostatic waves in ferromagnetic films [166]. The rigorous and accurate computation of the coefficients of a NLS equation for spatial solitons in a planar optical waveguide has allowed us to explain a polarization switching which was observed experimentally [168]. Recently, solitons in cylindrical magnetic nanowires have been described [169]. These development are very promising for both fundamental and applicative points of view.

\section{References}

[1] Russell J S 1844 Report on waves 14th Meeting of the British Association for the Advancement of Science

[2] Whitham V 1974 Linear and Nonlinear Waves (New York: Wiley-Interscience) 
[3] Boussinesq J 1872 Théorie des ondes et des remous qui se propagent le long d'un canal rectangulaire horizontal, en communiquant au liquide contenu dans ce canal des vitesses sensiblement pareilles de la surface au fond J. de Mathématiques Pures et Appliquées Sr. II 17 55-108

[4] Korteweg D J and de Vries G 1895 On the change of form of long waves advancing in a rectangular canal and on a new type of long stationary waves Phil. Mag. 5th series 39 422-43

[5] Gardner CS, Greene J M, Kruskal M D and Miura R M 1967 Method for solving the Korteweg-de Vries equation Phys. Rev. Lett. 19 1095-7

[6] Dodd R K, Eilbeck J C, Gibbon J D and Morris H C 1982 Solitons and Nonlinear Wave Equations (London: Academic)

[7] Ablowitz M J and Segur H 1981 Solitons and the Inverse Scattering Transform (Philadelphia, PA: SIAM)

[8] Lax P D 1968 Integrals of nonlinear equations of evolution and solitary waves Commun. Pure Appl. Math. 21 467-90

[9] Zakharov V E and Shabat A B 1972 Exact theory of two-dimensional self-focusing and one-dimensional self-modulation of waves in nonlinear media Sov. Phys.-JETP 34 62-9

[10] Mollenauer L F, Stolen R H and Gordon J P 1980 Experimental observation of picosecond pulse narrowing and solitons in optical fibers Phys. Rev. Lett. 45 1095-8

[11] Barthelemy A, Maneuf S and Froehly C 1985 Soliton propagation and self-confinement of laser-beams by Kerr optical non-linearity Opt. Commun. 55 201-6

[12] Zakharov V E and Kuznetsov E A 1986 Multi-scale expansions in the theory of systems integrable by the inverse scattering transform Physica D 18 455-63

[13] Gardner C S and Morikawa G K 1960 Similarity in the asymptotic behaviour of collision free hydromagnetic wave and water waves, New York University Report NYU-9082 Courant Institute of Mathematical Sciences (unpublished)

[14] Washimi H and Taniuti T 1966 Propagation of ion acoustic solitary waves of small amplitude Phys. Rev. Lett. 17 996-8

[15] Taniuti T and Wei C-C 1968 Reductive perturbation method in nonlinear wave propagation I J. Phys. Soc. Japan 24 941-6

[16] Su C H and Gardner C S 1969 Korteweg-de Vries equation and generalizations: III. Derivation of the Kortewegde Vries equation and Burgers equation J. Math. Phys. 10 536-9

[17] Taniuti T and Washimi H 1968 Self-trapping and instability of hydromagnetic waves along the magnetic field in a cold plasma Phys. Rev. Lett. 21 209-12

[18] Nakata I 1991 Weak nonlinear electromagnetic waves in a ferromagnet propagating parallel to an external field J. Phys. Soc. Japan 60 3976-7

[19] Taniuti T and Yajima N 1969 Perturbation method for a nonlinear wave modulation I J. Math. Phys. 10 1369-72

[20] Taniuti T and Yajima N 1973 Perturbation method for a nonlinear wave modulation III J. Math. Phys. 14 1389-97

[21] Hunter J K and Keller J B 1983 Weakly nonlinear high frequency waves Commun. Pure Appl. Math. 36 547-69

[22] Joly J L, Métivier G and Rauch J 1993 Resonant one dimensional nonlinear geometric optics J. Funct. Anal. 114 106-231

[23] Donnat P 1994 Quelques contributions mathématiques en optique non linéaire Thesis École Polytechnique

[24] Colin T 2002 Rigorous derivation of the nonlinear Schrödinger equation and Davey-Stewartson systems from quadratic hyperbolic systems Asymptot. Anal. 31 69-91
[25] Colin T and Lannes D 2004 Justification of and long-wave correction to Davey-Stewartson systems from quadratic hyperbolic systems Discrete Continuous Dyn. Syst. 11 83-100

[26] Colin T, Galusinski C and Kaper H G 2002 Long waves in micromagnetism Commun. PDE 27 1625-58, 3

[27] Leblond H and Manna M 1993 Coalescence of electromagnetic travelling waves in a saturated ferrite J. Phys. A: Math. Gen. 26 6451-68

[28] Boucher V and Nguyen Phu X 2002 Generation of multicolor spatial solitons by stimulated Raman scattering Appl. Opt. 41 21, 4390-3

[29] Boucher V 2001 Solitons spatiaux: contribution à l'étude de propagation non linéaire, $P h D$ Thesis University of Angers

[30] Joly J L, Métivier G and Rauch J 2000 Transparent nonlinear geometric optics and Maxwell-Bloch equations J. Diff. Eqns 166 175-250

[31] Leblond H 1995 Interaction of two solitary waves in a ferromagnet J. Phys. A: Math. Gen. 28 3763-84

[32] Leblond H 2002 Higher order terms in multiscale expansions: a linearised KdV hierarchy J. Nonlin. Math. Phys. $9325-46$

[33] Leblond H 2005 The KdV hierarchy and the propagation of solitons on very long distances Math. Comput. Simul. $69368-77$

[34] Miura R M 1968 Korteweg-de Vries equation and generalizations: I. A remarkable explicit nonlinear transformation J. Math. Phys. 9 1202-9

[35] Kaup D J, Reiman A and Bers A 1979 Space-time evolution of nonlinear three waves interactions: I. Interaction in a homogeneous medium Rev. Mod. Phys. 51 275-310

[36] Degasperis A, Conforti M, Baronio F and Wabnitz S 2006 Stable control of pulse speed in parametric three-wave solitons Phys. Rev. Lett. 97093901

[37] Conforti M, Baronio F, Degasperis A and Wabnitz S 2006 Inelastic scattering and interactions of three-wave parametric solitons Phys. Rev. E 74065602

[38] Zakharov V E and Manakov S V 1975 The theory of resonance interaction of wave packets in nonlinear media Sov. Phys.-JETP 42 842-50

[39] Stegeman G I $1997 \chi^{(2)}$ cascading: nonlinear phase shifts J. Opt. B: Quantum Semiclass. Opt. 9 139-53

[40] Leblond H 1995 Nonlinear anomalous ferromagnetic Faraday effect Phys. Rev. E 52 1081-93

[41] Leblond H 2002 Mixed perturbative expansion: the validity of a model for the cascading J. Nonlin. Math. Phys. 9 126-43

[42] Leblond H 2002 Parametric light bullets: a multiscale analysis J. Opt. A: Pure Appl. Opt. 4 160-73

[43] Boucher V, Leblond H and Nguyen Phu X 2002 Multiscale theory of nonlinear wave packet propagation in a planar optical waveguide J. Opt. A: Pure Appl. Opt. 4 514-20

[44] Leblond H 1995 Transition between stimulated backscattering and soliton exchange in ferrites J. Phys. A: Math. Gen. 28 2661-81

[45] Bergé L 1998 Wave collapse in physics: principles and applications to light and plasma waves Phys. Rep. 303 259-370

[46] Davey A and Stewartson K 1974 On three-dimensional packets of surface waves Proc. R. Soc. A 338 101-10

[47] Ablowitz M J and Clarkson P A 1991 Solitons, Nonlinear Evolution Equations and Inverse Scattering (Cambridge: Cambridge University Press)

[48] Leblond H 1998 Propagation of optical localised pulses in $\chi^{(2)}$ crystals: a $(3+1)$-dimensional model and its reduction to the NLS equation J. Phys. A: Math. Gen. 31 3041-66

[49] Leblond H 1999 Electromagnetic waves in ferromagnets: a Davey-Stewartson type model J. Phys. A: Math. Gen. 32 7907-32 
[50] Aguero M, Frantzeskakis D J and Kevrekidis P G 2006 Asymptotic reductions of two coupled $(2+1)$-dimensional nonlinear Schrödinger equations: application to BoseEinstein condensates J. Phys. A: Math. Gen. 39 7705-18

[51] Satsuma J and Ablowitz M J 1979 Two-dimensional lumps in nonlinear dispersive systems J. Math. Phys. 201496

[52] Boiti M, Leon J J-P, Martina L and Pempinelli F 1988 Scattering of localised solitons in the plane Phys. Lett. A 132 432-39

[53] Boiti M, Leon J and Pempinelli F 1990 Bifurcations of solitons in multidimensions Inverse Problems 6 715-23

Boiti M, Leon J and Pempinelli F 1990 Multidimensional solitons and their spectral transforms J. Math. Phys. $312612-8$

[54] Hietarinta J and Hirota R 1990 Multidromion solutions to the Davey-Stewarson equation Phys. Lett. A 145 237-44

[55] Taniuti T and Hasegawa Akira 1991 Reductive perturbation method for quasi one-dimensional nonlinear wave propagation: II. Applications to magnetosonic waves Wave Motion 13 133-46

[56] Kadomtsev B B and Petviashvili V I 1970 On the stability of solitary waves in weakly dispersing media Sov. Phys.-Dokl. 15 539-41

[57] de Bouard A and Saut J-C 1997 Symmetries and decay of the generalised Kadomtsev-Petviashvili solitary waves SIAM J. Math. Anal. 28 1064-85

[58] Kodama Y and Taniuti T 1978 Higher order approximation in the reductive perturbation method: I. The weakly dispersive system J. Phys. Soc. Japan $\mathbf{4 5} 298$

[59] Leblond H 1998 The secular solutions of the linearised KdV equation J. Math. Phys. 393772

[60] Kraenkel R A, Manna M A and Pereira J G 1995 The Korteweg-de Vries hierarchy and long water-waves J. Math. Phys. 36307

[61] Kraenkel R A, Manna M A, Montero J C and Pereira J G 1995 Boussinesq solitary wave as a multiple-time solution of the Korteweg-de Vries hierarchy J. Math. Phys. 366822

[62] Kraenkel R A, Manna M A and Pereira J G 1995 The reductive perturbation method and the Korteweg-de Vries hierarchy Acta Appl. Math. 39389

[63] Leblond H 2003 A new criterion for the existence of KdV solitons in ferromagnets J. Phys. A: Math. Gen. 36 1855-68

[64] Leblond H 2001 Solitons in ferromagnets and the KdV hierarchy Nonlinear Phenom. Complex Sys. 4 67-84

[65] Flaschka H, Newell A C and Tabor M 1991 Integrability What is Integrability? ed V E Zakharov (Berlin: Springer)

[66] Hirota R 1980 Direct method in soliton theory Solitons ed R K Bulough and P J Caudrey (Berlin: Springer)

[67] Cottam M G (ed) 1994 Linear and Nonlinear Spin Waves in Magnetic Films and Superlattices (Singapore: World Scientific)

[68] Wigen P E (ed) 1994 Nonlinear Phenomena and Chaos in Magnetic Material (Singapore: World Scientific)

[69] Kalinikos B A and Slavin A N 1986 Theory of dipole-exchange spin wave spectrum for ferromagnetic films with mixed exchange boundary conditions J. Phys. C: Solid State Phys. 19 7013-33

[70] De Gasperis P, Marcelli R and Miccoli G 1987 Magnetostatic soliton propagation at microwave frequency in magnetic garnet films Phys. Rev. Lett. 59 481-4

[71] Kalinikos B A, Kovshikov N G and Slavin A N 1990 Experimental observation of magnetostatic wave envelope solitons in yttrium iron garnet films Phys. Rev. B 42 8658-60

[72] Chen M, Tsankov M A, Nash J M and Patton C E 1993 Microwave magnetic-envelope dark-solitons in yttrium iron garnet thin films Phys. Rev. Lett. 70 1707-10
[73] Bauer M, Büttner O, Demokritov S O, Hillebrands B, Grimalsky V, Rapoport Yu and Slavin A N 1998 Observation of spatio-temporal self-focusing of spin-waves in magnetic films Phys. Rev. Lett. $813769-72$

[74] Büttner O, Bauer M, Demokritov S O, Hillebrands B, Kostylev M P, Kalinikos B A and Slavin A N 1999 Collisions of spin wave envelope solitons and self-focused spin-wave packets in yttrium iron garnet films Phys. Rev. Lett. 82 4320-3

[75] Temiryazev A G 1989 Modulational instability of electromagnetic-spin waves in ferrite film JETP Lett. 50 228-32

[76] Leblond H and Manna M 1994 Focusing and defocusing of electromagnetic waves in a ferromagnet J. Phys. A: Math. Gen. 27 3245-56

[77] Leblond H and Manna M 1994 Benjamin-Feir type instability in a saturated ferrite: transition between a focusing and defocusing regimen for polarised electromagnetic waves Phys. Rev. E 50 2275-86

[78] Slavin A N and Rojdestvenski I V 1994 'Bright' and 'dark' spin wave envelope solitons in magnetic films IEEE Trans. Magn. 30 37-45

[79] Benjamin T B and Feir J E 1967 The desintegration of wave trains in deep water: Part 1. Theory J. Fluid Mech. 27 417-30

[80] Zakharov V E and Shabat A B 1973 Interaction between solitons in a stable medium Sov. Phys._JETP 37 823-8

[81] Leblond H 1996 Electromagnetic waves in ferrites: from linear absorption to the nonlinear Schrödinger equation J. Phys. A: Math. Gen. 29 4623-39

[82] Zabolotskaya E A and Khokhlov R V 1969 Sov. Phys. Acoust. 835

[83] Murakami Y 1990 Obliquely interacting $N$-traveling waves. Exact solutions of some two-dimensional nonlinear diffusion equations J. Phys. Soc. Japan 59 1-4

[84] Sanchez D 2005 Long waves in ferromagnetic media, Khokhlov-Zabolotskaya equation J. Diff. Eqns $210263-289$

[85] Leblond H 2002 KP lumps in ferromagnets: a three-dimensional KdV-Burgers model J. Phys. A: Math. Gen. 35 10149-61

[86] Bass F G, Nasonov N N and Naumenko O V 1988 Dynamics of a Bloch wall in a magnetic field Sov. Phys. Tech. Phys. $33742-8$

[87] Landau L and Lifchitz E 1935 On the theory of dispersion of magnetic permeability in ferromagnetic bodies Phys. $Z$. Sowjet 8153 (reedition)

[88] Nakata I 1991 Nonlinear electromagnetic waves in a ferromagnet $J$. Phys. Soc. Japan $6077-81$

[89] Nakata I 1991 Shock waves in a ferromagnet J. Phys. Soc. Japan 60 2179-83

[90] Daniel M, Veerakumar V and Amuda R 1997 Soliton and electromagnetic wave propagation in a ferromagnetic medium Phys. Rev. E 55 3619-23

[91] Veerakumar V and Daniel M 1998 Electromagnetic soliton damping in a ferromagnetic medium Phys. Rev. E 57 1197-200

[92] Daniel M and Veerakumar V 2002 Propagation of electromagnetic soliton in antiferromagnetic medium Phys. Lett. A 302 77-86

[93] Veerakumar V and Daniel M 2001 Electromagnetic soliton in an anisotropic ferromagnetic medium under nonuniform perturbation Phys. Lett. A 278 331-8

[94] Leblond H 2000 Transverse stability of solitons and moving domain walls J. Phys. A: Math. Gen. 33 8105-26

[95] Ostrovskaia N V 2001 Magnetic solitons Soliton-Driven Photonics (NATO Science Series) ed A D Boardman and A P Sukhorukov (Dordrecht: Kluwer) 
[96] Leblond H 1996 Phase boom for an electromagnetic wave in a ferromagnet J. Phys. A: Math. Gen. 29 2805-25

[97] Volterra V 1913 Leçons sur les fonctions de lignes (Paris: Gauthier-Villars)

[98] Schetzen M 1980 The Volterra and Wiener Theories of Nonlinear Systems (New York: Wiley)

[99] Leblond H 2001 Direct derivation of a macroscopic NLS equation from the quantum theory J. Phys. A: Math. Gen. 34 3109-23

[100] Boyd R W 1992 Nonlinear Optics (San Diego, CA: Academic)

[101] Sutter D H et al 2000 Sub-6-fs pulses from a SESAM-assisted Kerr-lens mode locked Ti:sapphire laser: at the frontiers of ultrashort pulse generation Appl. Phys. B 70 S5-12

[102] Morgner U, Kärtner F X, Cho S H, Chen Y, Haus H A, Fujimoto J G, Ippen E P, Scheuer V, Angelow G and Tschudi T Sub-two-cycle pulses from a Kerr-lens mode-locked Ti:sapphire laser

[103] Ell R et al 2001 Generation of 5-fs pulses and octave-spanning spectra directly from a Ti:sapphire laser Opt. Lett. 26 373-5

[104] Brabec T and Krausz F 1997 Nonlinear optical pulse propagation in the single-cycle regime Phys. Rev. Lett. $783282-5$

[105] Blair S 2000 Non-paraxial 1-d spatial solitons Chaos 10570

[106] Leblond H and Sanchez F 2003 Models for optical solitons in the two-cycle regime Phys. Rev. A 67013804

[107] Wadati M 1973 The modified Korteweg-de Vries equation J. Phys. Soc. Japan 34 1289-96

[108] Hirota R 1976 Direct method of finding exact solutions of nonlinear evolution equations Bäcklund Transformations, the Inverse Scattering Method, Solitons, and Their Applications (Workshop Contact Transformation, Vanderbilt University, Nashville, TN, 1974) (Lecture Notes in Mathematics vol 515) (Berlin: Springer) pp 40-68

[109] Mel'nikov I V, Mihalache D, Moldoveanu F and Panoiu N-C 1997 Quasiadiabatic following of femtosecond optical pulses in a weakly excited semiconductor Phys. Rev. A 56 1569-76

[110] Manna M A and Merle V 1998 Asymptotic dynamics of short waves in nonlinear dispersive models Phys. Rev. E 576206

[111] Kraenkel R A, Manna M A and Merle V 2000 Nonlinear short-wave propagation in ferrites Phys. Rev. E 61 976-9

[112] Manna M A 2001 Nonlinear asymptotic short-wave models in fluid dynamics J. Phys. A: Math. Gen. 34 4475-91

[113] Leblond H, Sazonov S V, Mel'nikov I V, Mihalache D and Sanchez F 2006 Few-cycle nonlinear optics of multicomponent media Phys Rev. A $\mathbf{7 4} 063815$

[114] Yariv A and Yeh P 1983 Optical Waves in Crystals, Propagation and Control of Laser Radiation (New York: Wiley-Interscience)

[115] Stegeman G I 2001 Experiments in quadratic solitons Soliton-Driven Photonics (NATO Science Series) ed A D Boardman and A P Sukhorukov (Dordrecht: Kluwer)

[116] Menyuk C R, Schiek R and Torner L 1994 Solitary waves due to $\chi^{(2)}: \chi^{(2)}$ cascading J. Opt. Soc. Am. B $112434-43$

[117] Kaup D J, Malomed B A and Tasgal R S 1993 Internal dynamics of a vector soliton in a nonlinear optical fibre Phys. Rev. E 483049

[118] Haelterman M and Sheppard A P 1994 The elliptically polarised fundamental vector soliton of isotropic Kerr media Phys. Lett. A 194 191-6

[119] Manakov S V 1974 On the theory of two-dimensional stationary self-focusing of electromagnetic waves Sov. Phys._JETP 38248
[120] Zakharov V E and Schulman E I 1982 To the integrability of the system of two coupled nonlinear Schrödinger equations Physica D 4270

[121] Leblond H 1998 Bidimensional optical solitons in a quadratic medium J. Phys. A: Math. Gen. 31 5129-43

[122] Ablowitz M J, Biondini G and Blair S 2001 Nonlinear Schrödinger equations with mean terms in nonresonant multidimensional quadratic materials Phys. Rev. E $\mathbf{6 3} 046605$

[123] Dmitriev V G, Gurzadyan G G and Nikogosyan D N 1991 Handbook of Nonlinear Optical Crystals (Springer Series in Optical Sciences vol 64) (Berlin: Springer)

[124] Leblond H 2001 The Davey-stewartson model in quadratic media: a way to control pulses Soliton-Driven Photonics (NATO Science Series) ed A D Boardman and A P Sukhorukov (Dordrecht: Kluwer)

[125] Ghidaglia J M and Saut J C 1990 On the initial value problem for the Davey-Stewartson systems Nonlinearity 3 475-506

[126] Crasovan L-C, Torres J P, Mihalache D and Torner L 2003 Arresting wave collapse by wave self-rectification Phys. Rev. Lett. 91063904

[127] Leblond H 2005 Spatiotemporal optical pulse control using microwaves Phys. Rev. Lett. 95033902

[128] Malomed B A, Mihalache D, Wise F and Torner L 2005 Spatiotemporal optical solitons J. Opt. B 7 R53-72

[129] Baizakov B B, Malomed B A and Salerno M 2003 Multidimensional solitons in periodic potentials Europhys. Lett. 63 642-8

[130] Mihalache D, Mazilu D, Lederer F, Kartashov Y V, Crasovan L-C and Torner L 2004 Stable three-dimensional spatio-temporal solitons in a two-dimensional photonic lattice Phys. Rev. E 70055603

[131] Besse C and Bruneau C H 1998 Numerical study of elliptic-hyperbolic Davey-Stewartson system: dromions simulation and blow-up Math. Models Methods Appl. Sci. 8 1363-86

[132] Osborne A R and Petti M 1993 Numerical inversescattering-transform analysis of laboratory-generated surface wave trains Phys. Rev. E 47 1035-7

[133] Johnson R S 2003 The classical problem of water waves: a reservoir of integrable and nearly-integrable equations J. Nonlin. Math. Phys. 10 (Suppl 1) 72-92

[134] Benjamin T B, Bona J L and Mahony J J 1972 Model equations for long waves in nonlinear dispersive systems Phil. Trans. R. Soc. A 272 47-78

[135] Kruskal M 1975 Nonlinear wave equations, theory and applications Dynamical Systems, Theory and Applications (Lecture Notes in Physics vol 38) ed J Moser (New York: Springer) pp 310-54

[136] Bona J L, Pritchard W G and Scott L R 1983 A comparison of solutions of two model equations for long waves Proc. AMS-SIAM Conf. Fluid Dynamical Problems in Astrophysics and Geophysics (Chicago, July 1981) (Lectures in Applied Mathematics vol 20) ed N Lebovitz (Providence, RI: American Mathematical Society) pp 235-67

[137] Pouliquen O, Huerre P and Chomaz J-M 1995 Propagative sine-Gordon solitons in the spatially forced Kelvin-Helmholtz instability Physica D 80 333-55

[138] Kraenkel R A, Pereira J G and Manna M A 1992 Nonlinear surface-wave excitations in the Bénard-Marangoni system Phys. Rev. A 464786

[139] Kraenkel R A, Pereira J G and Manna M A 1992 Surface perturbations of a shallow viscous fluid heated from below and the (2+1)-dimensional Burgers equation Phys. Rev. A 45838

[140] Kraenkel R A, Manna M A and Pereira J G 1995 Modulational instability analysis of surface-waves in the 
Bénard-Marangoni phenomenon Physica D 87 356-60

[141] Manna M A and Neveu A 2001 Short-wave dynamics in the Euler equations Inverse Problems 17 855-61

[142] Manna M A 2001 Asymptotic dynamics of monochromatic short surface wind waves Physica D 149 231-6

[143] Kraenkel R A, Leon J and Manna M A 2005 Theory of small aspect ratio waves in deep water Physica D 211 377-90

[144] Hasegawa A and Tappert F 1973 Transmission of stationary nonlinear optical pulses in dispersive dielectric fibers: II. Normal dispersion Appl. Phys. Lett. 23 171-2

[145] Kivshar Y S 1991 Dark optical solitons near the zero-dispersion wavelength Opt. Lett. 16 892-4

[146] Frantzeskakis D J, Hizanidis K, Malomed B A and Polymilis C 1998 Stable anti-dark light bullets supported by the third-order dispersion Phys. Lett. A 248 203-7

[147] Bass F G, Konotop V V and Puzenko S A 1992 Dark solitary waves in a generalized version of the nonlinear Schrödinger equation Phys. Rev. A 46 4185-91

[148] Frantzeskakis D J 1997 Weak topological optical solitons in the femtosecond time scale J. Opt. Soc. Am. B 14 2359-64

[149] Kivshar Y 1990 Dark-soliton dynamics and shock waves induced by the stimulated Raman effect in optical fibers Phys. Rev. A 42 1757-61

[150] Kivshar Y S and Afanasjev V V 1991 Decay of dark solitons due to the stimulated Raman effect Opt. Lett. 16 285-7

[151] Frantzeskakis D J and Malomed B A 1999 Multiscale expansions for a generalized cylindrical nonlinear Schrödinger equation Phys. Lett. A 264 179-85

[152] Dreischuh A, Neshev D, Paulus G G, Grasbon F and Walther H 2002 Ring dark solitary waves: Experiment versus theory Phys. Rev. E 66066611

[153] Kivshar Y 1991 Dark optical solitons with reverse-sign amplitude Phys. Rev. A 44 R1446-9

[154] Nistazakis H E, Frantzeskakis D J and Malomed B A 2001 Collisions between spatiotemporal solitons of different dimensionality in a planar waveguide Phys. Rev. E 64026604

[155] Dalfovo F, Giorgini S, Pitaevskii L P and Stringari S 1999 Theory of Bose-Einstein condensation in trapped gases Rev. Mod. Phys. 71463
[156] Huang G, Szeftel J and Zhu1 S 2002 Dynamics of dark solitons in quasi-one-dimensional Bose-Einstein condensates Phys. Rev. A 65053605

[157] Huang G, Velarde M G and Makarov V A 2001 Dark solitons and their head-on collisions in Bose-Einstein condensates Phys. Rev. A 64013617

[158] Huang G, Makarov V A and Velarde M G 2003 Two-dimensional solitons in Bose-Einstein condensates with a disk-shaped trap Phys. Rev. A 67023604

[159] Huang G, Deng L and Hang C 2005 Davey-Stewartson description of two-dimensional nonlinear excitations in Bose-Einstein condensates Phys. Rev. E 72036621

[160] Kolomeisky E B, Newman T J, Straley J P and Qi X 2000 Low-dimensional Bose liquids: beyond the Gross-Pitaevskii approximation Phys. Rev. Lett. 851146

[161] Frantzeskakis D J, Proukakis N P and Kevrekidis P G 2004 Dynamics of shallow dark solitons in a trapped gas of impenetrable bosons Phys. Rev. A 70015601

[162] Frantzeskakis D J, Kevrekidis P G and Proukakis N P 2007 Crossover dark soliton dynamics in ultracold one-dimensional Bose gases Phys. Lett. A 364 129-34

[163] Kuznetsov E A and Turitsyn S K 1982 Two- and three-dimensional solitons in weakly dispersive media Sov. Phys. -JETP 55 844-7

[164] Manna M and Leblond H 2006 Transverse stability of short line-solitons in ferromagnetic media J. Phys. A: Math. Gen. 39 10437-47

[165] Stuart J T and DiPrima R C 1978 The Eckhaus and Benjamin-Feir resonance mechanisms Proc. R. Soc. A 362 27-41

[166] Leblond H 2001 Rigorous derivation of NLS in magnetic films J. Phys. A: Math. Gen. 34 9687-712

[167] Borzi C H, Kraenkel R A, Manna M A and Pereira A 2005 Nonlinear dynamics of short traveling capillary-gravity waves Phys. Rev. E 71026307

[168] Boucher V, Leblond H and Nguyen Phu X 2003 Polarization switching in a planar optical waveguide Phys. Rev. E 68056604

[169] Leblond H, Veerakumar V and Manna M 2007 Nonlinear spin waves in cylindrical ferromagnetic nanowires Phys. Rev. B 75214413 